JORGE CARVALHO COSTA

ESSENTIAL BOUNDARY AND INTERFACE CONDITIONS IN THE MESHLESS ANALYSIS OF SHELLS

São Paulo

2015 
JORGE CARVALHO COSTA

\section{ESSENTIAL BOUNDARY AND INTERFACE CONDITIONS IN THE MESHLESS ANALYSIS OF SHELLS}

Thesis submitted to the Polytechnic School at the University of São Paulo for award of the Doctor of Science Degree.

São Paulo 
JORGE CARVALHO COSTA

\section{ESSENTIAL BOUNDARY AND INTERFACE CONDITIONS IN THE MESHLESS ANALYSIS OF SHELLS}

Thesis submitted to the Polytechnic School at the University of São Paulo for award the Doctor of Science Degree.

Area: Structural Engineering

Advisor: Prof. Dr.Paulo de Mattos Pimenta

São Paulo 
Catalogação-na-publicação

\section{Costa, Jorge}

Essential boundary and interface conditions in the meshless analysis of shells / J. Costa -- São Paulo, 2015.

$63 \mathrm{p}$.

Tese (Doutorado) - Escola Politécnica da Universidade de São Paulo. Departamento de Engenharia de Estruturas e Geotécnica.

1.shells 2.meshless methods 3.nitsche's method 4.multi-region problems 5.shear deformable shells I.Universidade de São Paulo. Escola Politécnica. Departamento de Engenharia de Estruturas e Geotécnica II.t. 


\section{Essential boundary and interface conditions in the meshless analysis of shells}

Esta é a versão original da tese elaborada pelo candidato Jorge Carvalho Costa, tal como submetida à Comissão Julgadora. 


\section{Acknowledgments}

Firstly, I would like to thank Prof. Paulo Pimenta for taking me as a student and guiding me through my research and more importantly through a real academic formation. Thank you for the many fruitfull and happy get-togethers and conversations, about my research and a whole variaty of other subjects. It has been an honor to be your student.

Other masters were also kind enough to advise and help me, especially Prof. Carlos Tiago who welcomed me for a stay in Lisbon during my masters and became my referrence for all matters of meshless methods, and Prof. Eduardo Campello for great insights and helpful discussions.

My sincere gratitute to Prof. Peter Wriggers for receiving me at the Institut für Kontinuumsmechanik in Hannover for a prolific and very pleasant year. Thank you for helping my research and formation.

To my co-workers and office mates, thank you for providing a nice working environment, for attentive ears to obstacles and smily faces for the happy-hours. It was my privilege to work with Eduardo Simões, Paulo Nigro, Fernando Gonçalves, Marco Sampaio, Henrique Gomes, Leonardo Lago, Marcelo Teixeira, Igor Pierin, Nadia Ota, Alfredo Gay Neto, Márcia Sato, Alexandre Belleti, Luís Bitencourt, Ricardo Lahuerta at University of São Paulo, and to know some of their delightful families.

I also thank the new friends I made in Hannover, who made me feel welcome so far from home: Ajay Harish, Mohamed Anndif, Mohammadreza Hojjati, Sebastian Zeller, Saleh Yazdani, Vinh Hiep, Tobias Steiner and Christian Weißenfels among others at IKM. Also to Mrs. Halfar and Mrs. Schulte for all the help.

I was also lucky to find in Germany great new friends outside of the workplace. I thank Carlos Lopes, Melanie Titz and her many friends, Irene di Palma, Robin Corgier and Gabriela Szafarska, great flatmates, Mr. and Mrs. Franke. My best appreciation Rita Castro, Lüder Meyer and the Meyer family for such a great hospitality and also to the loving Totzke family.

My acknowledgment also go to CNPq (Conselho Nacional de Desenvolvimento Tecnológico) for a PhD scholarship and CAPES (Coordenação de Aperfeiçoamento de Pessoal de Nível Superior) for supporting my exchange stay at IKM with grant BEX 14618/13-3. 


\section{Agradecimentos}

É com um imenso prazer que agradeço a todos aqueles que caminharam junto comigo nessa jornada. Agradeço a Deus pelas oportunidades que me dá, pela minha paz e pelas pessoas que coloca junto a mim.

Agradeço a minha família por me dar segurança que o amor é grande e não conhece distâncias. A minhas avós Iza e Inês por todo carinho e por sempre rezarem por mim, as avós "emprestadas" Eunice e Nazinha por engrossarem esse coro, a meus avôs Joaldo e Lealdo (ambos in memorian) pelas recordaçoes e lições e também ao avô "emprestado" Osvaldo pelo carinho e as conversas sempre interessantes.

Dedico muito carinho a meus Tios e Tias que me guiam e suportam sempre. Em especial a minhas sempre presentes tias-mães Tereza e Marly, Rosa e Ângela; e tia Vânia, companheira de doutorado, que me incentiva e guia para a vida acadêmica, obrigado pela confiança. A meus tios João, Joca, Bôsco, Ricardo e Ivo por serem grandes exemplos. A meus primos-irmãos Joaldo, Evelyne e Izinha pelo apoio, companhia e amizade verdadeira. A meus primos-afilhados Vítor, Matheus, Rodrigo, Eduardo, Geovana e Thainá, que já cresceram, enjoaram das brincadeiras sem graça e se tornaram companheiros. A Jaiane e Léo, meus novos primos, e a Júlia por seu carinho sincero.

Também agradeço a minha família de Dores pela atenção e afeto, em espcial a Thiago pela torcida e apoio. Tudo que puder fazer por minha familia é pouco diante do que representa a mim.

Agradeço aos amigos que espalhei por aí e que me fazem sentir bem em todo lugar. A Alexandre, Anne, Walter e Íris pela paciência no dia a dia de quem mora junto. A Ryan, Moacir, Carlinhos, Yuri, Heloisa, Fabiana, Rodrigo Cocate e outras figuras paulistanas. A Ariel, Marcelinho, Aloísio, Samuel, Higor Sérgio, Marcel, Anísia, Thaísa, Gustavo, Juliana, Rony, Jorge, Fábio, Lucas, Diogo, Gugu, Madson, Flávio, Plínio e Ícaro. A Carlão Hermínio, Vítor e os outros 30 e tantos primos e tios.

Por fim, meu maior agradecimento a meus pais: Jorge Lima e Luzia. Obrigado pelo suporte, apoio, torcida, carinho e todas as formas de bem que sempre dirigiram a mim. Dedico esse trabalho a vocês. 


\section{Resumo}

Costa, J. C. . Condições essenciais de contorno e interface na análise de cascas com métodos sem malha. 2015. 63 f. Tese (Doutorado) - Escola Politécnica da Universidade de São Paulo, São Paulo, 2015.

Métodos sem malha geram campos de aproximação com alta continuidade, convenientes para estruturas finas como cascas. No entanto, a ausência da propriedade de Delta de Kronecker dificulta a formulação de condições essenciais de contorno, já que os campos de aproximação e teste não podem ser moldados aos valores de contorno. Um problema similar aparece quando diferentes regiões de aproximação precisam ser juntadas em um problema multi-regiões como dobras, vincos ou junções. Este trabalho apresenta três métodos de imposição ambas condições cinemáticas: o já conhecido método dos multiplicadores de Lagrange, usado desde o começo do método de Galekin sem elementos (EFG); uma abordagem de penalidade pura; e o recentemente redescoberto método de Nitsche. Nós usamos a técnica de discretização com EFG para cascas espessas de Reissner-Mindlin e adaptamos a forma fraca de forma a separar graus de liberdade de deslocamento e rotação e obter coeficientes de estabilização diferentes e apropriados. Essa abordagem permite a modelagem de cascas discontínuas e o refinamento local em problemas multi-regiões.

Palavras-chave: cascas, métodos sem malha, método de Nitsche, problemas multi-regiões, cascas com cisalhamento. 


\section{Abstract}

Costa, J. C. . Essential boundary and interface conditions in the meshless analysis of shells. 2015. 63 p. Thesis (Doctorate) - Escola Politécnica da Universidade de São Paulo, São Paulo, 2015.

Meshless methods provide a highly continuous approximation field, convenient for thin structures like shells. Nevertheless, the lack of Kronecker Delta property makes the formulation of essential boundary conditions not straightforward, as the trial and test fields cannot be tailored to boundary values. Similar problem arise when different approximation regions must be joined, in a multi-region problem, such as kinks, folds or joints. This work presents three approaches to impose both kinematic conditions: the well known Lagrange Multiplier method, used since the beginning of the Element Free Galerkin method; a pure penalty approach; and the recently rediscovered alternative of Nitsche's Method. We use the EFG discretization technique for thick Reissner-Mindlin shells and adapt the weak form as to separate displacement and rotational degrees of freedom and obtain suitable and separate stabilization parameters. This approach enables the modeling of discontinuous shells and local refinement on multi-region problems.

Keywords: shells, meshless methods, nitsche's method, multi-region problems, shear deformable shells. 
... and I have my mind ... and a mind needs books as a sword needs a whetstone, if it is to keep its edge

- George R. R. Martin 


\section{Contents}

1 Introduction 1

2 Linear shell theory $\quad 4$

2.1 Introduction . . . . . . . . . . . . . . . . . . 4

2.2 Kinematics . . . . . . . . . . . . . . . . . 4

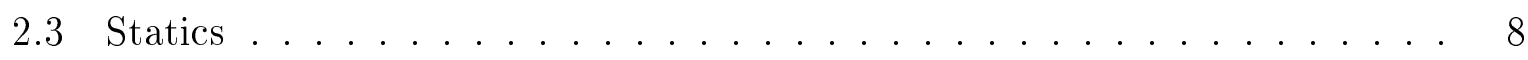

2.4 Linear approximation . . . . . . . . . . . . . . . 10

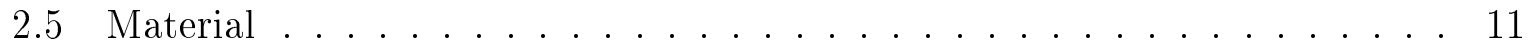

2.6 Initial Deformation . . . . . . . . . . . . . . . . 12

2.7 Weak Form of Equilibrium . . . . . . . . . . . . . . 13

2.8 Multi-region shells . . . . . . . . . . . . . . . . . 14

3 Meshless Approximation $\quad 16$

4 Kinematic Constraints 20

4.1 Lagrange Multipliers . . . . . . . . . . . . . . . . 22

4.2 Penalty Method . . . . . . . . . . . . . 27

4.3 Nitsche's Method . . . . . . . . . . . . . . . . . 31

5 Numerical Examples $\quad 41$

5.1 Stabilization for EBCs ....................... 41

5.1 .1 Displacement DOFs . . . . . . . . . . . . . . 41

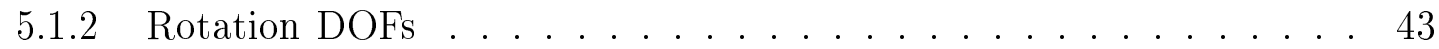

5.2 Stabilization for Interfaces . . . . . . . . . . . . . . . 45

5.2 .1 Displacement DOFs . . . . . . . . . . . . . 45

5.2 .2 Rotation DOFs . . . . . . . . . . . . . . . 47

5.3 Pinched Cylinder . . . . . . . . . . . . . . . . . 49

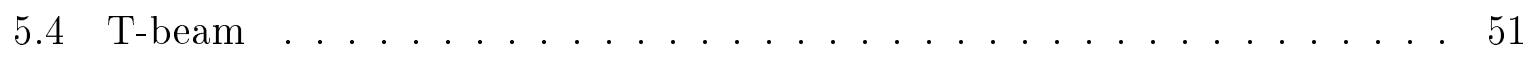

6 Conclusions $\quad 56$ 


\section{Chapter 1}

\section{Introduction}

Meshless approximations provide a great resource for the analysis of structures as high order continuity in the approximated fields can be achieved. This feature is well suited for thin systems like shells, provided that the geometry can be better approximated and stresses can be obtained as smoothly as desired. They have been applied to thin Kirchhoff-Love shells [37, 31], where $C^{1}$ continuity is necessary, moderately thick ReissnerMindlin shells [48, 58, 12] and solid-like shells based on 3D continuum [38]. However, the non-interpolatory characteristic of such approximants makes the formulation of essential boundary and interface conditions not straightforward.

We introduce interface conditions for continuity and equilibrium across a boundary between two discretization domains. The coupling of multiple regions is quite straightforward in Finite Elements (FE) with matching nodes along the interface, but this limits the liberty of each region's refinement. A growing application in Computer Aided Engineering is the possibility to analyze full, complex models composed of multiple parts, keeping the possibility to refine the response in most critical areas. In Computational Mechanics, this translates into the need for multiple regions to be describe together while maintaining the ability to be refined independently. One region might possibly need a more refined solution, or different approximation techniques might be employed in each side of the interface (i.e., FEs and meshless). Similar problem arise when dealing with approximations that do not fulfill the Kronecker delta property such as most meshless methods (meshless approximations on both domains). For mapped shell theories, each domain must also be smooth, so any kink or junction of shells is treated as a interface between independently approximated fields.

Essential boundary conditions (EBCs) and interface conditions (ICs) are formulated in the same way and have been discussed since the early development of meshless methods. From the beginning, as in the classical Element-Free Galerkin Method (EFG, [6]), Lagrange Multipliers have been used to enforce EBCs, but disadvantages like increasing the number of unknowns for the problem and limitations on the choice of approximation fields compelled more research on the subject $[35,18]$. Solutions ranged from coupling with FEs 
along the boundaries $[7,29]$ to the use of the domain shape functions to approximate the Lagrange multipliers [42, 43, 9].

Recently, an alternative has been revisited: the interior penalty method, usually referred to as Nitsche's Method [47], which identifies the Lagrange Multipliers with the flux at the essential or interface boundary and introduces a penalty-like stabilization parameter, which guarantees the coercivity of the bilinear form.

Among other applications, this approach has been used to impose EBCs in Poisson's problems with meshless methods [20, 18] and FEs [57]. It has been applied with eXtended Finite Elements (XFEM) for EBCs and ICs in single-field problems [15, 25, 28], to enforce weak discontinuities between elastic materials [51, 52, 2, 1]; and applied to non conforming embedded FE meshes for elastostatics [53, 54]. B-splines and NURBs-base FEs share similar features with meshless approximations and can also take advantage of Nitsche's Method, as in [16] for 2nd and 4th order problems, [46, 33, 49, 50] for elasticity, [27, 26] for thin plates and $[22,23]$ for thin shells. The theory has also been extended for contact with FEs [59] and XFEM [4, 3].

It is the authors' opinion that in the future Nitsche's method may become the standard treatment to enforcing essential boundary condition for meshfree Galerkin methods. [39, p. 114]

The present work develops Nitsche's method for the linear elastic analysis of shear deformable Reissner-Mindlin shells [12], both for the imposition of boundary displacements and for multi-region problems. In the latter, refinement over one portion of the domain can be performed without affecting other regions, even maintaining their stiffness matrices, and different regions can be discretized with different approximants, e.g., FE-EFG or EFG-IGA coupling.

In both cases, its advantage over Lagrange Multipliers is that it precludes the definition of a new approximation space, new degrees of freedom and a hybrid interpolation, along with its complications. A penalty-like stabilization term is necessary to ensure the coercivity of the bilinear form. Nevertheless, the weak form remains consistent and, contrary to a pure penalty imposition, accuracy is guaranteed for a wide range of values for the parameter.

The shear deformable shell theory is developed in [14] with Finite Elements and in [58] with Multiple Fixed Least Squares (MFLS). In [12], a consistent linear model is derived for small displacements and rotations, using MFLS and Lagrange multipliers. The extension to geometrically exact thin shells is present in [31] and the boundary conditions are discussed in [32]. This work presents the multi-region formulation for independent particle distributions, using both Lagrange multipliers and Interior Penalty for the interfaces. Numerical examples are laid out to assess the method's suitability, while comparing solutions with similar number of degrees of freedom. 
Throughout the text, italic Greek or Latin lowercase letters $(a, b, \ldots, \alpha, \beta, \ldots)$ denote scalar quantities; bold italic Greek or Latin lowercase letters $(\boldsymbol{a}, \boldsymbol{b}, \ldots, \boldsymbol{\alpha}, \boldsymbol{\beta}, \ldots)$ denote vectors and bold italic Greek or Latin capital letters $(\boldsymbol{A}, \boldsymbol{B}, \ldots, \boldsymbol{\Phi}, \boldsymbol{\Gamma}, \ldots)$ denote second-order tensors in a three-dimensional Euclidean space. Summation convention over repeated indices is adopted, with Greek indices ranging from 1 to 2 and Latin indices from 1 to 3 . 


\section{Chapter 2}

\section{Linear shell theory}

\subsection{Introduction}

The nonlinear shell theory developed in [14] has been later remodeled in a consistent way to a neat linear formulation in [12] to the first order in the displacements and rotations. This chapter outlines the process as in [10] so a consistent linear shell model is obtained.

The shell is parameterized by a flat reference configuration, consisting of a flat midsurface and a thickness $h$. The initial curved configuration is then obtained by a stress-free transformation after which the actual or effective transformation takes place. Fig. 2.1 depicts these transformations for the geometrically exact shell.

Let $\boldsymbol{e}_{i}$ be an orthogonal frame, with the vectors $\boldsymbol{e}_{\alpha}^{r}$ on the reference plane and the vector $\boldsymbol{e}_{3}^{r}$ orthogonal to it. At the reference configuration, a point of the shell can be described by its position

$$
\boldsymbol{\xi}=\boldsymbol{\zeta}+\boldsymbol{a}^{r}
$$

where $\boldsymbol{\zeta}=\xi_{\alpha} \boldsymbol{e}_{\alpha}^{r}$ defines the projection of this position in the midsurface and $\boldsymbol{a}^{r}=\xi_{3} \boldsymbol{e}_{3}^{r}$ is the through-the-thickness component. The set $\left[\xi_{1}, \xi_{2}, \xi_{3}\right]$ defines a Cartesian system of coordinates in three dimensions.

The shell's midsurface is the domain $\Omega^{r} \in \boldsymbol{e}_{1}^{r} \times \boldsymbol{e}_{2}^{r}$ and we assume that the midsurface is actually on the midpoint of the shell thickness $\xi_{3} \in[-h / 2, h / 2]$

\section{$2.2 \quad$ Kinematics}

The initial deformation is regarded as a mapping from the flat reference configuration. The position of the shell midsurface points is described in its initial stress-free configuration by

$$
\boldsymbol{z}^{o}=\boldsymbol{z}^{o}\left(\xi_{\alpha}\right)
$$




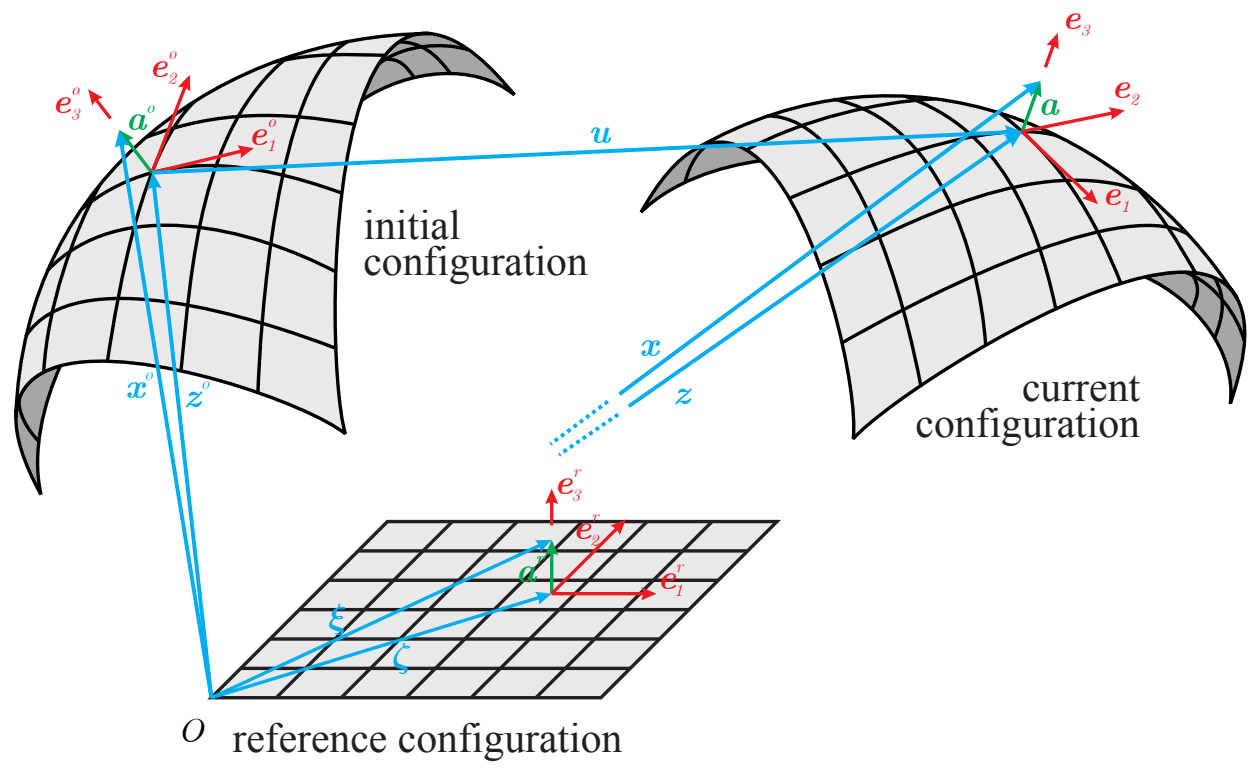

Figure 2.1: Shell kinematics for large displacements

These displacements are finite, and so are the rotations of the midsurface and the director. Nevertheless, Kirchhoff-Love kinematics is assumed, so that the director $\boldsymbol{a}^{o}$ remains normal to the curved midsurface. We can describe this rotation by the tensor $\boldsymbol{Q}^{o}$ So

$$
\boldsymbol{a}^{o}=\boldsymbol{Q}^{o} \boldsymbol{a}^{r}=\boldsymbol{Q}^{o} \xi_{3} \boldsymbol{e}_{3}^{r}
$$

The reference frame follows the same rotation,

$$
\boldsymbol{e}_{i}^{o}=\boldsymbol{Q}^{o} \boldsymbol{e}_{i}^{r}
$$

what makes it describable by

$$
\boldsymbol{Q}^{o}=\boldsymbol{e}_{i}^{o} \otimes \boldsymbol{e}_{i}^{r}
$$

As the initial frame $\boldsymbol{e}_{i}^{o}$ follows the midsurface curvature, it can be expressed of the derivatives of the initial position by the mapping coordinates:

$$
\begin{aligned}
\boldsymbol{e}_{1}^{o} & =\frac{\boldsymbol{z}_{, 1}^{o}}{\left\|\boldsymbol{z}_{, 1}^{o}\right\|}, \\
\boldsymbol{e}_{2}^{o} & =\boldsymbol{e}_{3}^{o} \times \boldsymbol{e}_{1}^{o} \text { and } \\
\boldsymbol{e}_{3}^{o} & =\frac{\boldsymbol{z}_{, 1}^{o} \times \boldsymbol{z}_{, 2}^{o}}{\left\|\boldsymbol{z}_{, 1}^{o} \times \boldsymbol{z}_{, 2}^{o}\right\|} .
\end{aligned}
$$

$\boldsymbol{e}_{1}^{o}$ and $\boldsymbol{e}_{2}^{o}$ are tangent to the shell surface and the director is $\boldsymbol{a}^{o}=\xi_{3} \boldsymbol{e}_{3}^{o}$. With the 
definition of the initial cross-sectional strain vectors

$$
\begin{aligned}
& \boldsymbol{\eta}_{\alpha}^{o}=\boldsymbol{z}_{\alpha}^{o}-\boldsymbol{Q}^{o} \boldsymbol{e}_{\alpha}^{r} \text { and } \\
& \boldsymbol{\kappa}_{\alpha}^{o}=\operatorname{axial}\left(\boldsymbol{Q}_{, \alpha}^{o} \boldsymbol{Q}^{o T}\right), \boldsymbol{K}_{\alpha}^{o}=\operatorname{Skew}\left(\boldsymbol{\kappa}_{\alpha}^{o}\right)
\end{aligned}
$$

at hand, the initial strain vectors at any material point can be computed by

$$
\gamma_{\alpha}^{o}=\boldsymbol{\eta}_{\alpha}^{o}+\kappa_{\alpha}^{o} \times \boldsymbol{a}^{o}
$$

so that the initial deformation gradient can be expressed by

$$
\boldsymbol{F}^{o}=\left(\boldsymbol{\eta}_{\alpha}^{o}+\boldsymbol{K}_{\alpha}^{o} \boldsymbol{a}^{o}\right) \otimes \boldsymbol{e}_{\alpha}^{r}+\boldsymbol{Q}^{o}=\boldsymbol{\gamma}_{\alpha}^{o} \otimes \boldsymbol{e}_{\alpha}^{r}+\boldsymbol{Q}^{o}
$$

The quantities above are affected by superposed rigid body motions and not suitable for an objective theory. Therefore, it's compelling to introduce their back-rotated counterparts as follows

$$
\begin{aligned}
\gamma_{\alpha}^{o r} & =\boldsymbol{Q}^{o T} \boldsymbol{\gamma}_{\alpha}^{o}=\boldsymbol{\eta}_{\alpha}^{o r}+\boldsymbol{\kappa}_{\alpha}^{o r} \times \boldsymbol{a}^{r} \\
\boldsymbol{\eta}_{\alpha}^{o r} & =\boldsymbol{Q}^{o T} \boldsymbol{\eta}_{\alpha}^{o}=\boldsymbol{Q}^{o T} \boldsymbol{z}_{, \alpha}^{o}-\boldsymbol{e}_{\alpha}^{r} \\
\boldsymbol{\kappa}_{\alpha}^{o r} & =\boldsymbol{Q}^{o T} \boldsymbol{\kappa}_{\alpha}^{o}=\left(\boldsymbol{e}_{2, \alpha}^{o} \cdot \boldsymbol{e}_{3}^{e}\right) \boldsymbol{e}_{1}^{r}+\left(\boldsymbol{e}_{3, \alpha}^{o} \cdot \boldsymbol{e}_{1}^{e}\right) \boldsymbol{e}_{2}^{r}+\left(\boldsymbol{e}_{1, \alpha}^{o} \cdot \boldsymbol{e}_{2}^{e}\right) \boldsymbol{e}_{3}^{r} \\
\boldsymbol{F}^{o r} & =\boldsymbol{Q}^{o T} \boldsymbol{F}^{o}
\end{aligned}
$$

For later use, it is convenient to gather the initial cross-sectional strain vectors in two generalized initial strain vectors

$$
\varepsilon_{\alpha}^{o r}=\left[\begin{array}{c}
\boldsymbol{\eta}_{\alpha}^{o r} \\
\boldsymbol{\kappa}_{\alpha}^{o r}
\end{array}\right] .
$$

Next, the actual motion of the shell takes place. The displacement of any point in the shell can be once again split into the displacement $\boldsymbol{u}$ of its projection on the midsurface and a rotation of the director $\boldsymbol{a}=\boldsymbol{Q}^{e} \boldsymbol{a}^{o}$. Since Reissner-Mindlin kinematics is assumed, the cross-section rotation is considered an independent quantity. The effective rotation $\boldsymbol{\theta}$ can be described by Euler parameters grouped in the rotation vector through the EulerRodrigues relation:

$$
\boldsymbol{Q}^{e}=\boldsymbol{I}+h_{1}(\theta) \boldsymbol{\Theta}+h_{2}(\theta) \boldsymbol{\Theta}^{2}
$$


where

$$
\begin{aligned}
\boldsymbol{\Theta} & =\text { Skew }(\theta), \\
\theta & =\|\boldsymbol{\theta}\|, \\
h_{1}(\theta) & =\frac{\sin (\theta)}{\theta}, \\
h_{2}(\theta) & =\frac{1}{2}\left(\frac{\sin \left(\frac{\theta}{2}\right)}{\frac{\theta}{2}}\right)^{2} .
\end{aligned}
$$

The current position of a material shell point can be expressed by

$$
\boldsymbol{x}=\boldsymbol{z}+\boldsymbol{a}=\boldsymbol{z}^{o}+\boldsymbol{u}+\boldsymbol{Q}^{e} \boldsymbol{a}^{o} .
$$

The components of $\boldsymbol{u}$ along with the components of $\boldsymbol{\theta}$ in a global Cartesian system define the six degrees of freedom of the theory.

The deformation from the flat reference configuration to the current deformed configuration is called the total deformation, and its gradient is given by

$$
\boldsymbol{F}=\frac{\partial \boldsymbol{x}}{\partial \boldsymbol{\xi}}=\boldsymbol{Q}+\left(\boldsymbol{\eta}_{\alpha}+\boldsymbol{\kappa}_{\alpha} \times \boldsymbol{a}\right) \otimes \boldsymbol{e}_{\alpha}^{r}
$$

where, as it was done for the initial deformation, one can define

$$
\begin{aligned}
\boldsymbol{\eta}_{\alpha} & =\boldsymbol{z}_{, \alpha}-\boldsymbol{e}_{\alpha}=\boldsymbol{z}_{, \alpha}^{o}+\boldsymbol{u}_{, \alpha}-\boldsymbol{Q} \boldsymbol{e}_{\alpha}^{r} \text { and } \\
\boldsymbol{\kappa}_{\alpha} & =\operatorname{axial}\left(\boldsymbol{Q}_{, \alpha} \boldsymbol{Q}^{T}\right)=\boldsymbol{\kappa}_{\alpha}^{e}+\boldsymbol{Q}^{e} \boldsymbol{\kappa}_{\alpha}^{o}
\end{aligned}
$$

as the cross-sectional strain vectors. Once again, their back-rotated counterparts are more suitable for an objective theory. After some algebra, we arrive at

$$
\begin{aligned}
\boldsymbol{\eta}_{\alpha}^{r} & =\boldsymbol{Q}^{T} \boldsymbol{\eta}_{\alpha}=\boldsymbol{Q}^{T} \boldsymbol{z}_{, \alpha}-\boldsymbol{e}_{\alpha}^{r} \text { and } \\
\boldsymbol{\kappa}_{\alpha}^{r} & =\boldsymbol{Q}^{T} \boldsymbol{\kappa}_{\alpha}=\boldsymbol{\kappa}_{\alpha}^{e r}+\boldsymbol{\kappa}_{\alpha}^{o r},
\end{aligned}
$$

where

$$
\begin{aligned}
\boldsymbol{\kappa}_{\alpha}^{e r} & =\boldsymbol{\Gamma}^{T} \boldsymbol{\theta}_{, \alpha}, \text { with } \\
\boldsymbol{\Gamma} & =\boldsymbol{I}+h_{2}(\theta) \boldsymbol{\Theta}+h_{3}(\theta) \boldsymbol{\Theta}^{2} \text { and } \\
h_{3}(\theta) & =\frac{1-h_{1}(\theta)}{\theta^{2}} .
\end{aligned}
$$

Finally the back-rotated strain vectors can be defined as

$$
\boldsymbol{\varepsilon}_{\alpha}^{r}=\left[\begin{array}{c}
\boldsymbol{\eta}_{\alpha}^{r} \\
\boldsymbol{\kappa}_{\alpha}^{r}
\end{array}\right],
$$


and the total back-rotated strains as

$$
\boldsymbol{\gamma}_{\alpha}^{r}=\boldsymbol{\eta}_{\alpha}^{r}+\boldsymbol{\kappa}_{\alpha}^{r} \times \boldsymbol{a}^{r}
$$

so the total deformation gradient can be expressed by

$$
\boldsymbol{F}=\boldsymbol{Q}\left(\boldsymbol{I}+\boldsymbol{\gamma}_{\alpha}^{r} \otimes \boldsymbol{e}_{\alpha}^{r}\right)
$$

Its back-rotated counterpart, not affected by rigid body motions is

$$
\boldsymbol{F}^{r}=\boldsymbol{Q}^{T} \boldsymbol{F}=\boldsymbol{I}+\boldsymbol{\gamma}_{\alpha}^{r} \otimes \boldsymbol{e}_{\alpha}^{r}
$$

This total deformation gradient can be decomposed into initial and effective ones

$$
\boldsymbol{F}=\boldsymbol{F}^{e} \boldsymbol{F}^{o}, \boldsymbol{F}^{e}=\boldsymbol{F} \boldsymbol{F}^{o-1}=\boldsymbol{Q}\left(\boldsymbol{f}_{i}^{e r} \otimes \boldsymbol{e}_{i}^{o}\right)
$$

The effective back-rotated strains can be defined as

$$
\gamma_{\alpha}^{e r}=\boldsymbol{f}_{\alpha}^{e r}-\boldsymbol{e}_{\alpha}^{r}=J^{o-1}\left(\boldsymbol{e}_{\alpha}^{r} \cdot \boldsymbol{g}_{\beta}^{o r}\right)\left(\boldsymbol{e}_{\beta}^{r}+\boldsymbol{\gamma}_{\beta}^{r}\right)-\boldsymbol{e}_{\alpha}^{r}
$$

where $\boldsymbol{g}_{\beta}^{o r}$ are derived from the initial transformation and $J^{o}$ is its Jacobian, as follows

$$
\begin{gathered}
J^{o}=\operatorname{det} \boldsymbol{F}^{o}=\operatorname{det} \boldsymbol{F}^{o}=\boldsymbol{e}_{3}^{r} \cdot \boldsymbol{f}_{1}^{o r} \times \boldsymbol{f}_{2}^{o r}, \\
\boldsymbol{g}_{1}^{o r}=\boldsymbol{f}_{2}^{o r} \times \boldsymbol{e}_{3}^{r}, \boldsymbol{g}_{2}^{o r}=\boldsymbol{e}_{3}^{r} \times \boldsymbol{f}_{1}^{o r}, \boldsymbol{g}_{3}^{o r}=\boldsymbol{f}_{1}^{o r} \times \boldsymbol{f}_{2}^{o r} .
\end{gathered}
$$

\subsection{Statics}

The shell specific internal power per unit volume at the reference configuration can be expressed by

$$
\boldsymbol{P}: \dot{\boldsymbol{F}}=J^{o} \boldsymbol{P}: \dot{\boldsymbol{F}}^{e}=\boldsymbol{\tau}_{\alpha}^{r} \cdot \dot{\boldsymbol{\eta}}_{\alpha}^{r}+\left(\boldsymbol{a}^{r} \times \boldsymbol{\tau}_{\alpha}^{r}\right) \cdot \dot{\boldsymbol{\kappa}}_{\alpha}^{r},
$$

where $\boldsymbol{P}=\boldsymbol{\tau}_{\alpha} \otimes \boldsymbol{e}_{\alpha}^{r}$ is the first Piolla-Kirchhoff stress tensor, $\boldsymbol{\tau}_{\alpha}^{r}=\boldsymbol{Q}^{o T} \boldsymbol{\tau}_{\alpha}$ are its backrotated column vectors and $\dot{\boldsymbol{F}}$ is the velocity gradient. Integration across the thickness yields the internal power per unit area of the midsurface in the initial configuration

$$
\int_{H}(\boldsymbol{P}: \dot{\boldsymbol{F}}) d H=\boldsymbol{n}_{\alpha}^{r} \cdot \dot{\boldsymbol{\eta}}_{\alpha}^{r}+\boldsymbol{m}_{\alpha}^{r} \cdot \dot{\boldsymbol{\kappa}}_{\alpha}^{r}
$$

where $H=\left[-{ }^{h} / 2,{ }^{h} / 2\right]$ is the thickness domain and the vectors

$$
\boldsymbol{n}_{\alpha}^{r}=\int_{H}\left(\boldsymbol{\tau}_{\alpha}^{r}\right) d H \text { and } \boldsymbol{m}_{\alpha}^{r}=\int_{H}\left(\boldsymbol{a}^{r} \times \boldsymbol{\tau}_{\alpha}\right) d H
$$


are the back-rotated force and moment resultants, respectively. Those can be gathered in the generalized back-rotated stress vectors

$$
\boldsymbol{\sigma}_{\alpha}^{r}=\left[\begin{array}{c}
\boldsymbol{n}_{\alpha}^{r} \\
\boldsymbol{m}_{\alpha}^{r}
\end{array}\right]
$$

so that the internal the internal power of the shell is

$$
P^{i n t}=\int_{V^{r}}(\boldsymbol{P}: \dot{\boldsymbol{F}}) d V^{r}=\int_{\Omega^{r}}\left(\boldsymbol{\sigma}_{\alpha}^{r} \cdot \dot{\boldsymbol{\varepsilon}}_{\alpha}^{r}\right) d \Omega^{r}
$$

The external power can be computed in the reference configuration by

$$
P^{e x t}=\int_{\Omega^{r}}\left(\overline{\boldsymbol{q}}^{\Omega^{r}} \cdot \dot{\boldsymbol{d}}\right) d \Omega^{r}+\int_{\Gamma_{t}^{r}}\left(\overline{\boldsymbol{q}}^{\Gamma_{t}^{r}} \cdot \dot{\boldsymbol{d}}\right) d \Gamma_{t}+\int_{\Gamma_{u}^{r}}\left(\boldsymbol{q}^{\Gamma_{u}^{r}} \cdot \dot{\boldsymbol{d}}\right) d \Gamma_{u}^{r}
$$

where

$$
\overline{\boldsymbol{q}}^{\Omega^{r}}=\left[\begin{array}{c}
\overline{\boldsymbol{n}}^{\Omega^{r}} \\
\boldsymbol{\Gamma}^{T} \overline{\boldsymbol{m}}^{\Omega^{r}}
\end{array}\right]
$$

are the force and moment resultants of the loads applied in the domain,

$$
\overline{\boldsymbol{q}}^{\Gamma_{t}^{r}}=\left[\begin{array}{c}
\overline{\boldsymbol{n}}^{\Gamma_{t}^{r}} \\
\boldsymbol{\Gamma}^{T} \overline{\boldsymbol{m}}^{\Gamma_{t}^{r}}
\end{array}\right]
$$

in the natural boundary and

$$
\boldsymbol{q}^{\Gamma_{u}^{r}}=\left[\begin{array}{c}
\boldsymbol{n}^{\Gamma_{u}^{r}} \\
\boldsymbol{\Gamma}^{T} \boldsymbol{m}^{\Gamma_{u}^{r}}
\end{array}\right]
$$

are the (unknown) reactions in the essential boundary $\Gamma_{u}^{r}$. Those (true) stress resultants are defined as

$$
\begin{aligned}
\overline{\boldsymbol{n}}^{\Omega^{r}} & =\overline{\boldsymbol{t}}^{t r}+\overline{\boldsymbol{t}}^{b r}+\int_{h} \overline{\boldsymbol{b}}^{r} d h \\
\overline{\boldsymbol{m}}^{\Omega^{r}} & =\boldsymbol{a} \times \overline{\boldsymbol{t}}^{b r}+\int_{h} \boldsymbol{a} \times \overline{\boldsymbol{b}}^{r} d h
\end{aligned}
$$

on the domain,

$$
\begin{aligned}
\overline{\boldsymbol{n}}^{\Gamma_{t}^{r}} & =\int_{h} \overline{\boldsymbol{t}}^{l r} d h \\
\overline{\boldsymbol{m}}^{\Gamma_{t}^{r}} & =\int_{h} \boldsymbol{a} \times \overline{\boldsymbol{t}}^{l r} d h
\end{aligned}
$$


on the Neumann boundary, and

$$
\begin{aligned}
\boldsymbol{n}^{\Gamma_{u}^{r}} & =\int_{h} \boldsymbol{r} d h \\
\boldsymbol{m}^{\Gamma_{u}^{r}} & =\int_{h} \boldsymbol{a} \times \boldsymbol{r} d h
\end{aligned}
$$

on the Dirichlet boundary.

In (2.35), $\overline{\boldsymbol{t}}^{t}, \overline{\boldsymbol{t}}^{b}, \overline{\boldsymbol{t}}^{l}$ are, respectively, the external surface tractions on the top, the bottom and the lateral of the shell, per unit reference area, $\bar{b}$ is the body force vector per unit reference volume and $\boldsymbol{r}$ is the reaction on the lateral surface at the kinematic boundary.

\subsection{Linear approximation}

The consistent linearization of the model to a first order in the displacements and rotations was carried out in [12]. The obtained model is similar to a thick plate theory, encompassing shell curvature and membrane deformation.

To the first order in $\boldsymbol{\theta}$, the displacement $\boldsymbol{\delta}$ during the effective transformation of a given point in the shell is

$$
\boldsymbol{\delta}=\boldsymbol{u}+\boldsymbol{\theta} \times \boldsymbol{a}^{o} .
$$

Back-rotated (objective) generalized membrane and curvature strain quantities [8, p. 2043] can be defined as the approximations of Eq. 2.17 to the first order in $\boldsymbol{u}$ and $\boldsymbol{\theta}$

$$
\begin{aligned}
\boldsymbol{\eta}_{\alpha}^{r} & =\boldsymbol{Q}^{o T}\left(\boldsymbol{z}_{, \alpha}^{o}+\boldsymbol{Z}_{, \alpha}^{o} \boldsymbol{\theta}+\boldsymbol{u}_{, \alpha}\right)-\boldsymbol{e}_{\alpha}^{r} \text { and } \\
\boldsymbol{\kappa}_{\alpha}^{r} & =\boldsymbol{Q}^{o T}\left(\boldsymbol{\kappa}_{, \alpha}^{o}+\boldsymbol{\theta}_{, \alpha}\right) .
\end{aligned}
$$

where

$$
\boldsymbol{Z}_{, \alpha}^{o}=\operatorname{Skew}\left(\boldsymbol{z}_{, \alpha}^{o}\right)
$$

It is interesting to note the neat additive property

$$
\begin{aligned}
\boldsymbol{\eta}_{\alpha}^{r} & =\boldsymbol{\eta}_{\alpha}^{o r}+\boldsymbol{\eta}^{e r}, \text { where } \\
\boldsymbol{\eta}_{\alpha}^{o r} & =\boldsymbol{Q}^{o T} \boldsymbol{z}_{, \alpha}^{o}-\boldsymbol{e}_{\alpha}^{r} \text { and } \\
\boldsymbol{\eta}_{\alpha}^{e r} & =\boldsymbol{Q}^{o T} \boldsymbol{Z}_{, \alpha}^{o} \boldsymbol{\theta}+\boldsymbol{Q}^{o T} \boldsymbol{u}_{, \alpha}
\end{aligned}
$$

and

$$
\begin{gathered}
\boldsymbol{\kappa}_{\alpha}^{r}=\boldsymbol{\kappa}_{\alpha}^{o r}+\boldsymbol{\kappa}^{e r}, \text { where } \\
\boldsymbol{\kappa}_{\alpha}^{o r}=\boldsymbol{Q}^{o T} \boldsymbol{\kappa}_{\alpha}^{o}=\left(\boldsymbol{e}_{2, \alpha}^{o} \cdot \boldsymbol{e}_{3}^{o}\right) \boldsymbol{e}_{1}^{r}+\left(\boldsymbol{e}_{3, \alpha}^{o} \cdot \boldsymbol{e}_{1}^{o}\right) \boldsymbol{e}_{2}^{r}+\left(\boldsymbol{e}_{1, \alpha}^{o} \cdot \boldsymbol{e}_{2}^{o}\right) \boldsymbol{e}_{3}^{r}
\end{gathered}
$$




$$
\boldsymbol{\kappa}_{\alpha}^{e r}=\boldsymbol{Q}^{o T} \boldsymbol{\theta}_{, \alpha}
$$

of the generalized strains. It's also compelling to split the generalized strain vectors $\varepsilon_{\alpha}^{r}$ given in (2.19) into initial and effective strains

$$
\varepsilon_{\alpha}^{r}=\varepsilon_{\alpha}^{o r}+\varepsilon_{\alpha}^{e r}
$$

The initial strains $\varepsilon_{\alpha}^{o r}$ are given by $(2.39 \mathrm{~b})$ and $(2.40 \mathrm{~b})$ and $\varepsilon_{\alpha}^{\text {er }}$ can be expressed in matrix for as

$$
\begin{gathered}
\boldsymbol{\varepsilon}_{\alpha}^{e r}=\boldsymbol{\Delta}_{\alpha} \boldsymbol{d} \\
\boldsymbol{\Delta}_{\alpha}=\left[\begin{array}{cc}
\boldsymbol{Q}^{o T} & \boldsymbol{O} \\
\boldsymbol{O} & \boldsymbol{Q}^{o T}
\end{array}\right]\left[\begin{array}{cc}
\boldsymbol{I} \frac{\partial}{\partial \boldsymbol{\xi}_{\alpha}} & \boldsymbol{Z}_{, \alpha}^{o} \\
\boldsymbol{O} & \boldsymbol{I} \frac{\partial}{\partial \boldsymbol{\xi}_{\alpha}}
\end{array}\right]
\end{gathered}
$$

where the shell's degrees of freedom are collected in

$$
\boldsymbol{d}=\left[\begin{array}{l}
\boldsymbol{u} \\
\boldsymbol{\theta}
\end{array}\right]
$$

\subsection{Material}

A generalized Hooke material is used. With the little abuse of nomenclature made in most of the consulted literature, the model presented here is said to be subject to a plane stress assumption. Actually, the model accounts for shear stresses in the thickness direction, what by itself is a violation of the plane stress condition. The assumption made herein is that there is no normal stress acting on the midsurface plane $\left(\boldsymbol{e}_{3}^{r} \cdot \boldsymbol{P}^{r} \boldsymbol{e}_{3}^{r}=0\right)$, as it has been done in [13] for the polyconvex neo-Hookean Simo-Ciarlet material (see [55], page 258). Thus, it is stated that

$$
\begin{gathered}
\boldsymbol{\tau}_{\alpha}^{e r}=\boldsymbol{C}_{\alpha \beta}^{e r} \boldsymbol{\gamma}_{\beta}^{e r}, \text { where } \\
\boldsymbol{C}_{\alpha \beta}^{e r}=\frac{E}{2(1-\nu)} \boldsymbol{e}_{\alpha}^{r} \otimes \boldsymbol{e}_{\beta}^{r}+\frac{E}{2(1+\nu)} \epsilon_{\alpha \beta} \boldsymbol{E}_{3}^{r}+\frac{E}{2(1+\nu)} \delta_{\alpha \beta} \boldsymbol{I},
\end{gathered}
$$

which is the isotropic plane stress generalized Hooke's law in our notation. In (2.44b), the skew-symmetric tensor $\boldsymbol{E}_{3}^{r}=\operatorname{Skew}\left(\boldsymbol{e}_{3}^{r}\right)$, the Kronecker symbol $\delta_{\alpha \beta}$ and the permutation symbol $\epsilon_{\alpha \beta}=\boldsymbol{e}_{\alpha}^{r} \cdot \boldsymbol{e}_{\beta}^{r} \times \boldsymbol{e}_{3}^{r}$ have been introduced. The linear elastic relation between the generalized back-rotated stresses $\boldsymbol{\sigma}_{\alpha}^{r}$ and the generalized effective strains $\varepsilon_{\alpha}^{e r}$ can be written as displayed below.

$$
\boldsymbol{\sigma}_{\alpha}^{r}=\boldsymbol{D}_{\alpha \beta} \varepsilon_{\beta}^{e r}=\boldsymbol{D}_{\alpha \beta}\left(\varepsilon_{\beta}^{r}-\varepsilon_{\beta}^{o r}\right)
$$




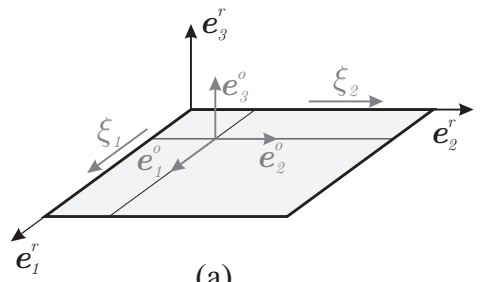

(a)

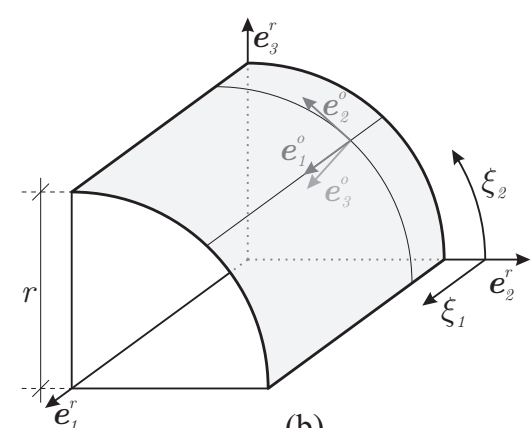

(b)

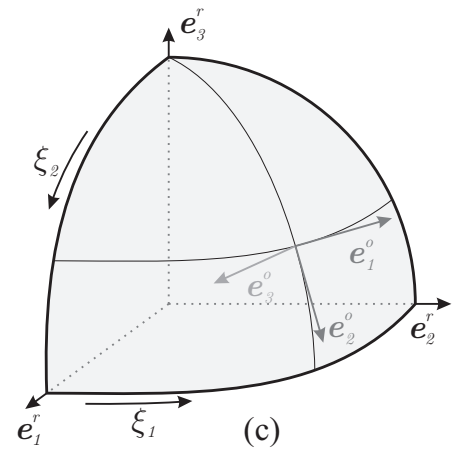

(c)

Figure 2.2: Initial deformations (a) flat shell, (b) cylindrical shell and (c) spherical shell

where

$$
\boldsymbol{D}_{\alpha \beta}=\left[\begin{array}{cc}
\boldsymbol{D}_{\alpha \beta}^{\eta \eta} & \boldsymbol{D}_{\alpha \beta}^{\eta \kappa} \\
\boldsymbol{D}_{\alpha \beta}^{\kappa \eta} & \boldsymbol{D}_{\alpha \beta}^{\kappa \kappa}
\end{array}\right]
$$

with

$$
\begin{aligned}
\boldsymbol{D}_{\alpha \beta}^{\eta \eta} & =\int_{H} \frac{1}{J^{o}}\left(\boldsymbol{g}_{\alpha}^{o r} \cdot \boldsymbol{e}_{\delta}^{r}\right) \boldsymbol{C}_{\delta \gamma}^{e r}\left(\boldsymbol{e}_{\gamma}^{r} \cdot \boldsymbol{g}_{\beta}^{o r}\right) d \xi_{3}, \\
\boldsymbol{D}_{\alpha \beta}^{\eta \kappa} & =-\int_{H} \frac{1}{J^{o}}\left(\boldsymbol{g}_{\alpha}^{o r} \cdot \boldsymbol{e}_{\delta}^{r}\right) \boldsymbol{C}_{\delta \gamma}^{e r}\left(\boldsymbol{e}_{\gamma}^{r} \cdot \boldsymbol{g}_{\beta}^{o r}\right) \boldsymbol{A}^{r} d \xi_{3} \\
& =\boldsymbol{D}_{\alpha \beta}^{\kappa \eta T} \text { and } \\
\boldsymbol{D}_{\alpha \beta}^{\kappa \kappa} & =\int_{H} \frac{1}{J^{o}} \boldsymbol{A}^{r}\left(\boldsymbol{g}_{\alpha}^{o r} \cdot \boldsymbol{e}_{\delta}^{r}\right) \boldsymbol{C}_{\delta \gamma}^{e r}\left(\boldsymbol{e}_{\gamma}^{r} \cdot \boldsymbol{g}_{\beta}^{o r}\right) \boldsymbol{A}^{r} d \xi_{3} .
\end{aligned}
$$

In (2.47) the skew symmetric tensor $\boldsymbol{A}_{3}^{r}=\operatorname{Skew}\left(\boldsymbol{a}^{r}\right)$ has been introduced and $\boldsymbol{g}_{\alpha}^{\text {or }}$ are the column vectors of the inverse of the transformation gradient $\boldsymbol{F}_{i}^{\text {or }} \otimes \boldsymbol{e}_{i}^{r}$ given in Eq. 2.25b.

\subsection{Initial Deformation}

The evaluation of the through the thickness integrals in the material matrix can be numerically performed for any initial mapping, including parametric approximations such as NURBS or T-splines. This can be avoided for some initial shell shapes through an analytical evaluation. Some of the obtained expressions can be further simplified if a thinness assumption is introduced, which is here defined by the condition $\frac{h}{R} \ll 1$, i.e. the initial curvature radius $R$ of the shell is much bigger than its thickness $h$. In [12], those integrations are done for the plane, cylindrical and spherical initial shapes depicted in Fig. 2.2. The resulting expressions for the sub-matrices in (2.47), common to all explicit 
and numerical mappings are

$$
\begin{aligned}
& \boldsymbol{D}_{\alpha \beta}^{\eta \eta}=h J^{o-1}\left(\boldsymbol{g}_{\alpha}^{o r} \cdot \boldsymbol{e}_{\delta}^{r}\right)\left(\boldsymbol{e}_{\gamma}^{r} \cdot \boldsymbol{g}_{\beta}^{o r}\right) \boldsymbol{C}_{\delta \gamma}^{e r}, \\
& \boldsymbol{D}_{\alpha \beta}^{\eta \kappa}=\boldsymbol{D}_{\alpha \beta}^{\kappa \eta T}=\boldsymbol{O} \text { and } \\
& \boldsymbol{D}_{\alpha \beta}^{\kappa \kappa}=-\frac{h^{3}}{12} J^{o-1}\left(\boldsymbol{g}_{\alpha}^{o r} \cdot \boldsymbol{e}_{\delta}^{r}\right)\left(\boldsymbol{e}_{\gamma}^{r} \cdot \boldsymbol{g}_{\beta}^{o r}\right) \boldsymbol{E}_{3}^{r} \boldsymbol{C}_{\delta \gamma}^{e r} \boldsymbol{E}_{3}^{r},
\end{aligned}
$$

with $J^{o}$ and $\boldsymbol{g}_{\alpha}^{o r}$ evaluated on the shell midsurface.

\subsection{Weak Form of Equilibrium}

Equilibrium can be asserted in a weak form as stating that the difference between internal and external works is zero. We can derive the internal virtual work from (2.30) as

$$
\delta W^{i n t}=\int_{\Gamma_{u}^{r}} \boldsymbol{\sigma}_{\alpha}^{r} \cdot \delta \varepsilon_{\alpha}^{r} d \Omega^{r} .
$$

From the external power (2.31), we separate the contributions of the external loads into the external virtual work

$$
\delta W^{e x t}=\int_{\Omega^{r}} \overline{\boldsymbol{q}}^{\Omega^{r}} \cdot \delta \boldsymbol{d} d \Omega^{r}+\int_{\Gamma_{t}^{r}} \overline{\boldsymbol{q}}^{\Gamma_{t}^{r}} \cdot \delta \boldsymbol{d} d \Gamma_{t}^{r}
$$

and the external virtual work of the reaction forces

$$
\delta W^{r e a}=\int_{\Gamma_{u}^{r}} \boldsymbol{q}^{\Gamma_{u}^{r}} \cdot \delta \boldsymbol{d} d \Gamma_{u}^{r}
$$

That separation will come in handy when describing the imposition of essential boundary conditions. Equilibrium can than be stated in a weak form as

$$
\delta W^{r e g}=\delta W^{i n t}-\delta W^{e x t}-\delta W^{r e a}=0 .
$$

Or in a more expressive way as "find $\boldsymbol{d} \in \mathscr{H}^{1}(\Omega)$ such that $\boldsymbol{d}=\overline{\boldsymbol{d}}$ in $\Gamma_{d}$ and

$$
\begin{aligned}
\delta W^{r e g} & =\int_{\Omega^{r}} \boldsymbol{\sigma}_{\alpha}^{r} \cdot \delta \varepsilon_{\alpha}^{r} d \Omega^{r}-\int_{\Omega^{r}} \overline{\boldsymbol{q}}^{\Omega^{r}} \cdot \delta \boldsymbol{d} d \Omega^{r} \\
& -\int_{\Gamma_{t}^{r}} \overline{\boldsymbol{q}}^{\Gamma_{t}^{r}} \cdot \delta \boldsymbol{d} d \Gamma_{t}^{r}-\int_{\Gamma_{u}^{r}} \boldsymbol{q}^{\Gamma_{u}^{r}} \cdot \delta \boldsymbol{d} d \Gamma_{u}^{r}=0
\end{aligned}
$$

for all $\delta \boldsymbol{d} \in \mathscr{H}^{1}(\Omega)$ ". It is important to note that using a non-interpolatory approximation $\delta \boldsymbol{d}$ cannot be made to vanish on the essential boundary, thus the last term (in $\Gamma_{u}^{r}$ ) is not canceled like in a Finite Element framework.

This imposition is linear on the generalized displacements as the stresses $\boldsymbol{\sigma}_{\alpha}^{r}$ are linearly dependent on the strains $\varepsilon_{\alpha}^{r}$ according to (2.45) and $\delta \varepsilon_{\alpha}^{r}=\boldsymbol{\Delta}_{\alpha} \delta \boldsymbol{d}$ is the variation of (2.41) 


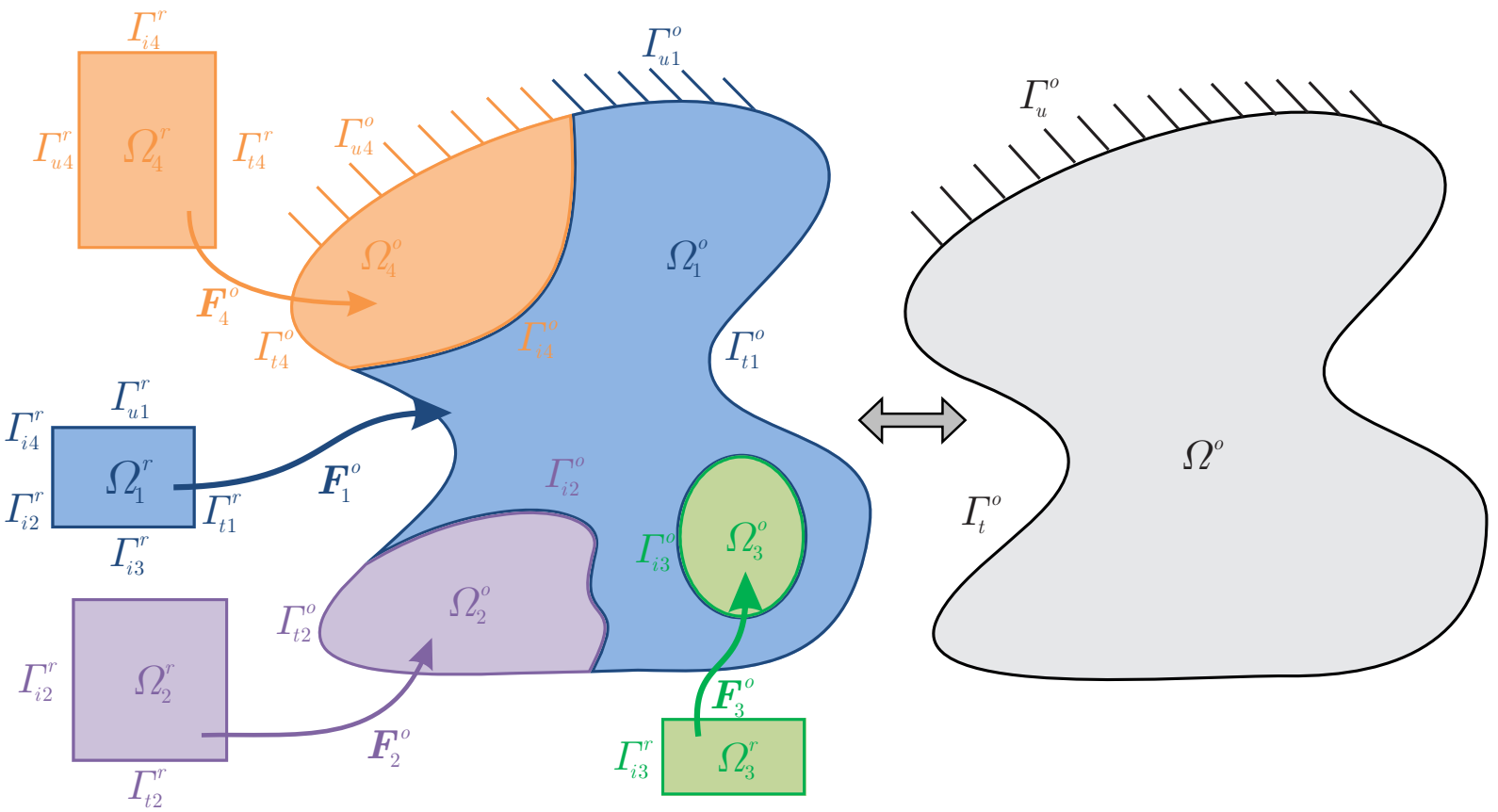

Figure 2.3: Multi-region problem.

using (2.42).

\subsection{Multi-region shells}

A multi-region problem arises when the problem domain cannot be represented by a single approximation region for lacking material continuity, usually due to change in material parameters such as Young modulus or thickness; or when different approximation properties are required, as coupling different approximation methods (e.g. EFG coupled to FEs, or Isogeometric NURBS) or different approximation properties like polynomial base or sudden particle density. In mapped shell analysis the initial configuration comes from a Kirchhoff-Love mapping, which require the shell to be continuous with also continuous first derivatives. The presence of folds or the connection of multiple shells along a line poses a material discontinuity, and the model should encompass these jumps. The approximation should then be continuous for displacements and rotations, but discontinuous for the generalized strains. This is said to be a weak discontinuity, as opposed to a strong discontinuity on the displacement fields.

Let the initial domain $\Omega^{o}$ in Fig. 2.3 be split into $n_{\text {reg }}$ multiple regions $\Omega_{n}^{o}$. Each region will have boundaries $\Gamma_{u n}^{o}$ where it meets the Dirichlet boundary, $\Gamma_{t n}^{o}$ along its intersection with the Newman boundary and interface boundary $\Gamma_{i n}^{o}$ where it contacts other regions (i.e. $\Omega_{1}^{o}$ ). Regions may have more than one interface boundary, or interact with other region rather than $\Omega_{1}^{o}$, but in order to keep a simple notation, those easily derivable cases will be omitted.

Each region comes by a different initial transformation $\boldsymbol{F}_{n}^{o}$ from a reference configu- 
ration $\Omega_{n}^{r}$. Our shell formulation performs integrations over these reference domains and the respective boundaries $\left(\Gamma_{u n}^{r}, \Gamma_{t n}^{r}\right.$ and $\left.\Gamma_{i n}^{r}\right)$. An interface refers to the line where different shells' midsurfaces intersect and the interface condition is the compatibility of the displacements and rotations coming from the approximations in both regions

$$
\left.\boldsymbol{d}\right|_{\Omega_{1}}=\left.\boldsymbol{d}\right|_{\Omega_{2}}
$$

and the equilibrium between the generalized stresses normal to the interface in both regions.

$$
\left.\boldsymbol{\sigma}_{n}\right|_{\Omega_{1}}=\left.\boldsymbol{\sigma}_{n}\right|_{\Omega_{2}}
$$




\section{Chapter 3}

\section{Meshless Approximation}

Among the many meshless approximation schemes described for instance in [39, 40] , the Moving Least Squares (MLS) will be used in the numerical examples. Still, the shell model and consequent discrete system of equations may be used with any field approximation scheme, especially non-interpolatory ones.

The so called Diffuse Approximation was used by Nayroles at al. [45] for plane elasticity problems. The seminal paper [6] identifies the formulation as the same approximation used for scattered data fitting by Lancaster and Salkauskas, McLain, Gordon and Wixom, Barnhill among others, called Moving Least Squares.

May $u(\boldsymbol{x})$ be a field variable to be approximated over $\Omega$. In the MLS framework, its approximation $u^{h}$ around a point $\boldsymbol{x} \in \Omega$ is expressed by a linear combination of set of functions, the basis $\boldsymbol{p}(\boldsymbol{x})$ :

$$
u^{h}(\boldsymbol{x})=\boldsymbol{p}(\boldsymbol{x}) \cdot \boldsymbol{a}(\boldsymbol{x}),
$$

with coefficients $\boldsymbol{a}$ that vary across the domain. If a set of particles $\overline{\boldsymbol{x}}_{i}$, positions over the domain or close to it, is associated to a set of nodal values $\overline{\boldsymbol{u}}_{i}$, the coefficients $\boldsymbol{a}(\boldsymbol{x})$ can be obtained through the minimization of the weighted error

$$
\begin{aligned}
J(\boldsymbol{a}) & =\sum_{i=1}^{n} w(r)\left(\bar{u}_{i}-u^{h}\left(\overline{\boldsymbol{x}}_{i}\right)\right)^{2}, \\
r & =\frac{\|\boldsymbol{x}-\overline{\boldsymbol{x}}\|}{r_{\max }}
\end{aligned}
$$

with respect to $\boldsymbol{a}$, where $w$ is a weighting function that enforces the local character of the approximation. It is usually a bell-shaped function with support radius 1 , that monotonically decreases from the unit at $r=0$ to zero when $r \geq 1$. The requirements for this function can be found along with examples in [58]. For the present context, it suffices 
to say that the fifth order spline

$$
w(r)= \begin{cases}1-10 r^{2}+20 r^{3}-15 r^{4}+4 r^{5} & \text { if } r \leq 1 \\ 0, & \text { if } r>1\end{cases}
$$

was chosen. The radius $r_{\max }$ characterizes the support of $w(r)$ at point $\overline{\boldsymbol{x}}_{i}$.

The minimization of (3.2) renders the linear system of equations

$$
A^{x} a^{x}=B^{x} \bar{u}
$$

where

$$
\begin{aligned}
\boldsymbol{A}^{x} & =\sum_{i=1}^{n} w\left(r_{i}\right) \boldsymbol{p}\left(\overline{\boldsymbol{x}}_{i}\right) \boldsymbol{p}\left(\overline{\boldsymbol{x}}_{i}\right)^{T} \\
\boldsymbol{B}^{\boldsymbol{x}} & =\left[\begin{array}{ll}
\boldsymbol{B}_{1}^{x} & \boldsymbol{B}_{2}^{x} \cdots \boldsymbol{B}_{n}^{x}
\end{array}\right] \\
\boldsymbol{B}_{\boldsymbol{i}}^{x} & =w\left(r_{i}\right) \boldsymbol{p}\left(\overline{\boldsymbol{x}}_{i}\right) .
\end{aligned}
$$

$\boldsymbol{B}_{i}^{x}$ is the $i^{\text {th }}$ column of $\boldsymbol{B}^{x}$ and $\boldsymbol{A}^{x}$ is usually known as the moment matrix. The approximation can then be expressed as

$$
u^{h}(\boldsymbol{x})=\sum_{i=1}^{n} \phi_{i}(\boldsymbol{x}) \bar{u}_{i}
$$

where $\phi_{i}(\boldsymbol{x})=\boldsymbol{p}(\boldsymbol{x}) \boldsymbol{A}^{-1}(\boldsymbol{x}) \boldsymbol{B}_{i}(\boldsymbol{x})$ is the shape function associated to the $i$ particle evaluated at point $\boldsymbol{x}$. The shape functions can be gathered in the vector $\boldsymbol{\Phi}(\boldsymbol{x})$, so the approximation is expressed in the standard way by

$$
u^{h}(x)=\boldsymbol{\Phi}(\boldsymbol{x}) \overline{\mathbf{u}},
$$

where $\overline{\mathbf{u}}$ gathers the approximation coefficients.

Usually, the number of particles in the support $n$ is higher than the size of the polynomial basis $\boldsymbol{p}(\boldsymbol{x})$. Thus $\boldsymbol{B}^{\boldsymbol{x}}$ is not square and $\boldsymbol{a}^{\boldsymbol{x}}$ has a different size than $\overline{\boldsymbol{u}}$. This minimization leads to functions that do not obey the Kronecker Delta property $\boldsymbol{\Phi}_{i}\left(\boldsymbol{x}_{i}\right)=\delta_{i j}$ or $u^{h}\left(\boldsymbol{x}_{i}\right)=\overline{\boldsymbol{u}}_{i}$.

The MLS are said to be an approximation without an interpolatory characteristic, and this will have major repercussions in the imposition of essential conditions (prescribed displacements) and continuity across different regions (kinks).

A distinction must be made as to how the weight function is defined. More specifically, how its parameters are stored. The MLS formulation presented requires the weight function (actually the support size parameter) to be defined in every interest point, i.e., 
quadrature sample, force application, result output points. In most found applications, the support for an interest point is not defined; instead each particle has its influence region limited by a support radius.

In [41], this distinction is made in the nomenclature of the domain sub-regions. If either the interest point support domain or the node influence domain is defined. In [58, 12], the former is called MLS and the latter Multiple Fixed Least Squares (MFLS). Other texts usually don't make this distinction, describing the approximation as MLS but implementing MFLS. For the numerical examples shown, the MFLS were used, with the nodal influence domains being defined by the weight function maximum radius $r_{\max }$.

This value is computed for each particle so that the approximation is well defined at every interest point, i.e., the moment matrix (3.6a) can be inverted. An semi-automatic procedure to determine the support radius for each particle was put in place. A set is made with the position of all interest points: domain and boundary integration points, force application points, output sample points, where the moment matrix will need to be inverted. A loop through these interest points make sure that each is enclosed in at least $(m+1)(m+2) / 2$ influence domains, where $m$ is the number of elements of the $2 \mathrm{D}$ polynomial base. If not enough particles define the approximation there, the radia corresponding to the closest particles whose supports do not include the interest point are increased. Nevertheless, particle alignment and other geometrical combinations might render linear dependencies. To ameliorate that, an amplification factor (1.75 unless stated otherwise) is applied to $r_{\max }$, which also serves to improve smoothness.

Throughout the text, we adopt the following approximation conventions. Suppose we use $n p$ particles to approximate each generalized displacement field. We approximate the displacements and rotations with MFLS as

$$
\boldsymbol{d}(\boldsymbol{x})=\boldsymbol{\Phi}(\boldsymbol{x}) \mathbf{d},
$$

and most of the time omit the dependencies on $\boldsymbol{x}$.

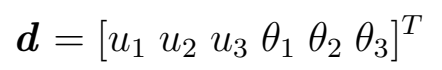

gathers the domain degrees of freedom. The matrix

$$
\boldsymbol{\Phi}=\left[\begin{array}{llll}
\Phi_{1} \mathbf{I}_{6} & \Phi_{2} \mathbf{I}_{6} & \cdots & \Phi_{n} \mathbf{I}_{6}
\end{array}\right]
$$

where $\mathbf{I}_{6}$ is the identity matrix of order 6 , distributes the shape functions and $\mathbf{d}$ collects the 6 approximation coefficients per particle. A reordering of the strain components allows 
for more useful expressions

$$
\begin{aligned}
& \boldsymbol{\eta}^{e r}=\left[\begin{array}{c}
\boldsymbol{\eta}_{1}^{e r} \\
\boldsymbol{\eta}_{2}^{e r}
\end{array}\right]=\left[\begin{array}{c}
\boldsymbol{\Delta}_{1}^{\eta} \boldsymbol{\Phi} \\
\boldsymbol{\Delta}_{2}^{\eta} \boldsymbol{\Phi}
\end{array}\right] \mathbf{d}=\mathbf{B}^{\eta} \mathbf{d}, \text { and } \\
& \boldsymbol{\kappa}^{e r}=\left[\begin{array}{c}
\boldsymbol{\kappa}_{1}^{e r} \\
\boldsymbol{\kappa}_{2}^{e r}
\end{array}\right]=\left[\begin{array}{c}
\boldsymbol{\Delta}_{1}^{\kappa} \boldsymbol{\Phi} \\
\boldsymbol{\Delta}_{2}^{\kappa} \boldsymbol{\Phi}
\end{array}\right] \mathbf{d}=\mathbf{B}^{\kappa} \mathbf{d} .
\end{aligned}
$$

Matrices $\mathbf{B}$ gather the derivatives of the shape functions as to obtain the generalized strains from the nodal degrees of freedom.

The Lagrange Multipliers, when used, must be defined along a line (a boundary or an interface). We use piece-wise linear interpolation for each component and gather the shape functions in matrix $\Psi$. The approximated fields are then

$$
\begin{aligned}
& \boldsymbol{q}^{\Gamma_{u}^{r}}=\boldsymbol{\Psi} \boldsymbol{\lambda}_{u}, \text { on the kinematic boundary } \\
& \boldsymbol{q}^{\Gamma_{i}^{r}}=\boldsymbol{\Psi} \boldsymbol{\lambda}_{i}, \text { on the interfaces. }
\end{aligned}
$$

Vectors $\boldsymbol{\lambda}_{u}$ and $\boldsymbol{\lambda}_{i}$ group respectively degrees of freedom on $\Gamma_{u}^{r}$ and $\Gamma_{i}^{r}$. The corresponding virtual quantities are approximated likewise.

$$
\begin{aligned}
& \delta \boldsymbol{q}^{\Gamma_{u}^{r}}=\boldsymbol{\Psi} \delta \boldsymbol{\lambda}_{u} \\
& \delta \boldsymbol{q}^{\Gamma_{i}^{r}}=\boldsymbol{\Psi} \delta \boldsymbol{\lambda}_{i} .
\end{aligned}
$$




\section{Chapter 4}

\section{Kinematic Constraints}

Chapter 3 described the Moving Least Squares shape functions used in the Elementfree Galerkin (EFG) Method. Like many of other meshless approximation schemes, MLS lacks the Kronecker Delta property, what makes the direct imposition of boundary values unpractical. As the test functions cannot be made zero along the essential boundary, term comprising the virtual work of the reaction forces cannot be omitted from Eq. 2.53, and as the trial space cannot be tailored to prescribed displacements and rotations, compatibility should be imposed otherwise.

Fernández-Méndez and Huerta [18] classify the imposition of EBCs in the EFG in two categories: modifications on the approximation shape functions and on the weak form, both approaches pursued in parallel during the development of meshless methods. A first example for modifying the weak form is Belytschko at al. [6], text that coined the term "Element-free Galerkin" and makes use of Lagrange Multipliers. A text by the same group [42] identifies the Lagrange Multipliers as the tractions on the boundary and adopts the same approximation used for the primary field to interpolate these tractions. This modified variational principle is very similar to a weak form derived by Nitsche [47] but for a stabilization term. Later [43], the trace of the domain shape functions was used to describe the Lagrange Multipliers on the boundary, also avoiding new degrees of freedom. These techniques couldn't reproduce the results obtained from Lagrange Multipliers, probably because the bilinear form lost its coercivity.

Modifications on the shape function are mostly of two kind. The meshless characteristic of the approximation is retained, such as in the Point Interpolation Method [41] or the approximation is blended into Finite Elements near the boundary, either for imposing EBCs or to join differently approximated domains. The merge is carried out with ramp functions [7, 35] or accounting for the FE shape function in the MLS reproducibility imposition ([29, 30] for EFG and [17] for Smooth Particle Hydrodynamics). Only the FE shape functions would have support over the boundaries and the EBCs would be imposed as such. [18] compares the techniques of modifying the shape function (bridging scales and continuous blending methods) and three possible modifications on the weak form: 
Lagrange Multipliers, Penalty and a revamped Nitsche's Method.

A similar imposition must be made when material properties change in the domain. In shell analysis, this would be characterized by shells with different thickness, materials, a sudden change in the shell curvature (folds or kinks) or the connection of more than two shells. In the formulation of the mapped shell problem, the initial mapping $\Omega^{r} \rightarrow \Omega^{o}$ is assumed to be of Kirchhoff-Love type and continuous, so a fold or connection, as a sudden change in this mapping, represents a transition of material. The solution field should then be continuous with discontinuous first derivatives. This is the typical nature of a finite element trial space if the mesh conforms to the boundary, as the shape functions are piecewise polynomials, but with discontinuous derivatives along the element edges.

Nevertheless, non-matching meshes cannot enforce continuity in a straightforward manner so many works are devoted to the use of Finite Elements for multiple regions. In [19], a comparison between Lagrange Multipliers and interior penalty is carried for linear elasticity, using multi-grids in nonconforming meshes, while [24] apply them to heat conduction, developing a priori and a posteriori error estimates. [53] uses the three approaches presented in this section for immersed boundaries (fictitious domain) in 2D elasticity problems. The domains can also be attached by coupling finite elements [34] with large aspect ratios that model the interaction between the domains.

Usual meshless functions are smooth across the domain. A high concentration of particles close to the interface area can provide a very steep gradient in the solution, but not exactly reproduce the jump. The process is quite similar to imposing essential boundary conditions, the displacement of the intersecting regions must be made equal and the stresses normal to the interface boundary compatible, so the solutions found are also similar to those used for EBCs, either modifying the shape function or adding contributions to the weak form.

Cordes and Moran [9] truncates the weight function of each node for the domain it is in. This corresponds to using different sets of nodes for the approximation of each domain, but for the nodes resting on the boundary, whose support stretches both domains. In $[5,36]$, the shape functions are enriched with a new term with the desired properties, in a similar procedure to eXtended Finite Elements (XFEM).

XFEM is another field in which such impositions must be made, as the boundaries and interfaces are not required to conform with the mesh and may cut through elements. In [28] Nitsche's method is derived from a Augmented Lagrange standpoint for an idealized diffusion problem. In [15], it is used for jump and Dirichlet problems in linear elasticity, with a more precise way to evaluate the flux on the interface and to determine the stabilization parameter in each element.

The method is also applied to spline-based approximations. The Finite Cell method consists of using FEs with spline base functions in a background mesh with immersed boundaries. Nitsche's method is than used to impose EBCs [49] and ICs [50] in two and 
three dimensional elasticity and is successful used to describe thin and solid-like shells $[22,21,23]$

This chapter focus on modifications of the weak form to impose EBCs and ICs between pairs of approximation domains in a shell model framework, retaining the MLS approximant in its most usual form for each domain. The next sections present the development needed for each type of modification of the weak form, recovering the original strong form whenever possible. Emphasis is given to Nitsche's Method, also known as Interior Penalty Method, as it is a new application for shells using EFG. The modification of the weak form takes the form of added virtual works on the kinematic boundary $\left(\delta W^{u}\right)$ and interfaces $\left(\delta W^{i}\right)$, so the final weak form is

$$
\delta W=\sum_{r e g} \delta W^{r e g}-\delta W^{u}-\sum_{i n t} \delta W^{i}
$$

For the sake of simplicity, we will retain our descriptions to a case with two approximation regions $\left(\Omega^{r}{ }_{1}\right.$ and $\left.\Omega^{r}{ }_{2}\right)$, with the essential boundary entirely contained on the limits of $\Omega^{r}{ }_{1}$. Extension to different situations is straightforward. We describe both types of imposition together to keep notation as simple as possible and the terms corresponding to EBCs and ICs resembles each other. Eq. (4.1) can then be expanded to

$$
\begin{gathered}
\delta W=\delta W_{1}^{i n t}+\delta W_{2}^{i n t}-\delta W^{e x t} \\
-\delta W^{r e a}-\delta W^{u}-\delta W^{i}=0 .
\end{gathered}
$$

The first three terms are defined by Eqs. 2.49-2.50 and the last three will be defined for each method.

\subsection{Lagrange Multipliers}

The Element-free Galerkin method is a development from the Diffuse Approximation introduced by Nayroles el al. [45]. One of the advances presented in [6] was the correct imposition of EBCs, along with corrections on the shape function derivatives and on the order of the integration quadrature. The earlier work used direct imposition on boundary nodes values, which is not sufficient for a non-interpolatory approximation. Lagrange multipliers were then introduced as to impose EBCs via a modification of the weak form.

The multipliers can be identified with the boundary reactions $\boldsymbol{q}^{\Gamma_{u}^{r}}$, approximated independently from the domain fields. Their contribution to the virtual work $\delta W^{\text {rea }}$ is kept as stated in Eq. 2.51 and their complementary virtual work is added as a weak imposition of the EBCs that also keeps the bilinear form symmetric.

$$
\delta W_{l a g}^{u}=\int_{\Gamma_{u}^{r}} \delta \boldsymbol{q}^{\Gamma_{u}^{r}} \cdot(\boldsymbol{d}-\overline{\boldsymbol{d}}) d \Gamma_{u}^{r}=0, \forall \delta \boldsymbol{q}^{\Gamma_{u}^{r}}
$$


It is important to note that the choice of the interpolation space for $\boldsymbol{q}^{\Gamma_{u}^{r}}$ and consequently $\delta \boldsymbol{q}^{\Gamma_{u}^{r}}$ must be careful. The problem becomes a saddle point problem, stable only if the approximated fields, e.g., $\boldsymbol{q}^{\Gamma_{u}^{r}}$ and $\boldsymbol{d}$ obey an inf-sup Babuska-Brezzi stability condition. A poor approximation space for $\boldsymbol{q}^{\Gamma_{u}^{r}}$ may not impose the boundary conditions with satisfactory accuracy while a richer space may render the resulting system of equations singular.

In a similar fashion, Interface conditions can be imposed. The generalized tractions normal to the interfaces $\boldsymbol{q}^{\Gamma_{i}^{r}}$ must be approximated independently from the displacements. A gap along the interface boundary would mean an additional potential

$$
\Pi_{l a g}^{i}=\int_{\Gamma_{i}^{r}} \boldsymbol{q}^{\Gamma_{i}^{r}} \cdot\left(\boldsymbol{d}_{1}-\boldsymbol{d}_{2}\right) d \Gamma_{i}^{r},
$$

where $\boldsymbol{d}_{1}$ and $\boldsymbol{d}_{2}$ are the displacements obtained by different region's approximation. The variation of (4.4) yields

$$
\begin{array}{r}
\delta W_{l a g}^{i}=\int_{\Gamma_{i}^{r}} \delta \boldsymbol{q}^{\Gamma_{i}^{r}} \cdot\left(\boldsymbol{d}_{1}-\boldsymbol{d}_{2}\right) d \Gamma_{i}^{r}+\int_{\Gamma_{i}^{r}} \boldsymbol{q}^{\Gamma_{i}^{r}} \cdot\left(\delta \boldsymbol{d}_{1}-\delta \boldsymbol{d}_{2}\right) d \Gamma_{i}^{r} \\
=0, \forall \delta \boldsymbol{q}^{\Gamma_{i}^{r}}, \delta \boldsymbol{d}_{1}, \delta \boldsymbol{d}_{2}
\end{array}
$$

which is the weak imposition of the continuity across the interface boundary using Lagrange Multipliers. The same observations made about the choice of the approximation space for $\boldsymbol{q}^{\Gamma_{u}^{r}}$ are valid for choosing the approximation space for $\boldsymbol{q}^{\Gamma_{i}^{r}}$, with the aggravating fact that a typical multi-region problem have interface boundaries $\Gamma_{i}^{r}$ longer than $\Gamma_{u}^{r}$.

The sum of the virtual works arising from all region's domain, the essential boundaries and interfaces gives the final weak form of the problem. For the Lagrange Multipliers, one should substitute (2.53), (4.3) and (4.5) into (4.1). If we define the jump operator [.] $=(.)_{\Omega^{r}{ }_{1}}-(.)_{\Omega^{r}{ }_{2}}$ on $\Gamma_{i}^{r}$ as the difference on the values obtained for the same field, at a same point on an interface, but using different domain approximation spaces, and $[]=.(.)_{\Omega^{r}}-\left({ }^{-}\right)$on $\Gamma_{u}^{r}$ as the difference between the quantity evaluated from the domain approximation and the prescribed value on the essential boundary, we can write the complete weak form for Lagrange Multipliers

$$
\begin{aligned}
\delta W_{\text {lag }} & =\sum_{n_{r e g}} \int_{\Omega^{r}} \delta \boldsymbol{\varepsilon}_{\alpha}^{r} \cdot \boldsymbol{\sigma}_{\alpha}^{r} d \Omega^{r}-\sum_{n_{r e g}} \int_{\Omega^{r}} \delta \boldsymbol{d} \cdot \overline{\boldsymbol{q}}^{\Omega^{r}} d \Omega^{r}-\int_{\Gamma_{t}^{r}} \delta \boldsymbol{d} \cdot \overline{\boldsymbol{q}}^{\Gamma_{t}^{r}} d \Gamma_{t}^{r} \\
& -\int_{\Gamma_{u}^{r}} \delta \boldsymbol{d} \cdot \boldsymbol{q}^{\Gamma_{u}^{r}} d \Gamma_{u}^{r}-\int_{\Gamma_{u}^{r}} \delta \boldsymbol{q}^{\Gamma_{u}^{r}} \cdot[\boldsymbol{d}] d \Gamma_{u}^{r} \\
& -\int_{\Gamma_{i}^{r}} \delta \boldsymbol{q}^{\Gamma_{i}^{r}} \cdot[\boldsymbol{d}] d \Gamma_{i}^{r}-\int_{\Gamma_{i}^{r}}[\delta \boldsymbol{d}] \cdot \boldsymbol{q}^{\Gamma_{i}^{r}} d \Gamma_{i}^{r} \\
& =0, \forall \delta \boldsymbol{d}, \delta \boldsymbol{q}^{\Gamma_{u}^{r}}, \delta \boldsymbol{q}^{\Gamma_{i}^{r}}
\end{aligned}
$$


After the discretization with nodal-based functions through Eqs. 3.9, 3.12 and 3.13, and choosing alternate unitary nodal virtual displacements, the linear system of equations can be obtained.

$$
\left[\begin{array}{cccc}
\mathbf{K}_{1} & \mathbf{0} & \mathbf{G}_{1}^{u} & \mathbf{G}_{1}^{i} \\
\mathbf{0} & \mathbf{K}_{2} & \mathbf{0} & \mathbf{G}_{2}^{i} \\
\mathbf{G}_{1}^{u T} & \mathbf{0} & \mathbf{0} & \mathbf{0} \\
\mathbf{G}_{1}^{i T} & \mathbf{G}_{2}^{i T} & \mathbf{0} & \mathbf{0}
\end{array}\right]\left[\begin{array}{c}
\mathbf{d}_{1} \\
\mathbf{d}_{2} \\
\boldsymbol{\lambda}^{u} \\
\boldsymbol{\lambda}^{i}
\end{array}\right]=\left[\begin{array}{c}
\mathbf{f}_{1} \\
\mathbf{f}_{2} \\
\mathbf{q}^{u} \\
\mathbf{0}
\end{array}\right]
$$

where

$$
\mathbf{K}_{n}=\int_{\Omega_{n}^{r}} \mathbf{B}_{\alpha}^{T} \mathbf{D}_{\alpha \beta} \mathbf{B}_{\beta} d \Omega_{n}^{r}
$$

is the stiffness matrix for each region,

$$
\mathbf{G}_{1}^{u}=-\int_{\Gamma_{u}^{r}} \boldsymbol{\Phi}_{1}^{T} \Psi d \Gamma_{u}^{r}
$$

imposes the EBCs and

$$
\begin{aligned}
\mathbf{G}_{1}^{i} & =-\int_{\Gamma_{i}^{r}} \boldsymbol{\Phi}_{1}^{T} \boldsymbol{\Psi} d \Gamma_{i}^{r}, \\
\mathbf{G}_{2}^{i} & =\int_{\Gamma_{i}^{r}} \boldsymbol{\Phi}_{2}^{T} \boldsymbol{\Psi} d \Gamma_{i}^{r} .
\end{aligned}
$$

impose the ICs. The force vectors are

$$
\begin{aligned}
\mathbf{f}_{n} & =\mathbf{f}^{\Omega_{n}^{r}}+\mathbf{f}^{\Gamma_{t n}^{r}} \\
\mathbf{f}^{\Omega^{r}} & =\int_{\Omega^{r}} \boldsymbol{\Phi}^{T} \overline{\boldsymbol{q}}^{\Omega^{r}} d \Omega^{r} \text { in the domain, } \\
\mathbf{f}^{\Gamma_{t}^{r}} & =\int_{\Gamma_{t}^{r}} \boldsymbol{\Phi}^{T} \overline{\boldsymbol{q}}^{\Gamma_{t}^{r}} d \Gamma_{t}^{r} \text { on the natural boundary, and } \\
\mathbf{q}_{\text {lag }}^{u} & =-\int_{\Gamma_{u}^{r}} \boldsymbol{\Psi} \overline{\boldsymbol{d}} d \Gamma_{u}^{r} \text { on the essential boundary. }
\end{aligned}
$$

The vectors $\mathbf{d}_{n}$ are the degrees of freedom used to approximate the displacements and rotations in region $n, \boldsymbol{\lambda}^{u}$ and $\boldsymbol{\lambda}^{i}$ are the degrees of freedom used to approximate the Lagrange Multipliers on the Dirichlet and interface boundaries respectively.

We should emphasize the block of zeros in the lower-right corner. The dimension of this block relates to the dimension of the Lagrange Multipliers approximation spaces. The matrix is symmetric, but not positive-definite, so a proper solver for linear systems of equations must be chosen.

MATLAB [44] have been used for the numerical examples in this work and the method for solving the system was kept transparent as it falls away from the scope of this article. 
Nevertheless, the solver warned for matrices close to singular or badly scaled, with a low condition number specially for models with long boundaries and interfaces, when a trivial approximation space was used for the Lagrange Multipliers (i.e. one node for each row of particles in that direction in the domain). This emphasizes the need for discretion on the choice of approximation spaces for this method.

The equilibrium equations for the original problem can be recovered from the final weak form (4.6). Using the strain definitions (2.37) and integrating by parts, the first term is

$$
\begin{aligned}
\delta W^{i n t} & =\int_{\Omega^{r}} \delta \varepsilon_{\alpha}^{r} \cdot \boldsymbol{\sigma}_{\alpha}^{r} d \Omega^{r}=\int_{\Omega^{r}} \delta \boldsymbol{\eta}_{\alpha}^{r} \cdot \boldsymbol{n}_{\alpha}^{r}+\boldsymbol{\kappa}_{\alpha}^{r} \cdot \boldsymbol{m}_{\alpha}^{r} d \Omega^{r} \\
& =\int_{\Omega^{r}} \boldsymbol{Q}^{o T}\left(\boldsymbol{Z}_{, \alpha}^{o} \delta \boldsymbol{\theta}+\delta \boldsymbol{u}_{, \alpha}\right) \cdot \boldsymbol{n}_{\alpha}^{r}+\boldsymbol{Q}^{o T} \delta \boldsymbol{\theta}_{, \alpha} \cdot \boldsymbol{m}_{\alpha}^{r} d \Omega^{r} \\
& =\int_{\Omega^{r}} \boldsymbol{Q}^{o T}\left(\boldsymbol{Z}_{, \alpha}^{o} \delta \boldsymbol{\theta}+\delta \boldsymbol{u}_{, \alpha}\right) \cdot \boldsymbol{Q}^{o T} \boldsymbol{n}_{\alpha}+\boldsymbol{Q}^{o T} \delta \boldsymbol{\theta}_{, \alpha} \cdot \boldsymbol{Q}^{o T} \boldsymbol{m}_{\alpha} d \Omega^{r} \\
& =\int_{\Omega^{r}} \boldsymbol{Z}_{, \alpha}^{o} \delta \boldsymbol{\theta} \cdot \boldsymbol{n}_{\alpha} d \Omega^{r}+\int_{\Gamma^{r}} \delta \boldsymbol{u} \cdot \boldsymbol{n}_{n} d \Gamma^{r}-\int_{\Omega^{r}} \delta \boldsymbol{u} \cdot \boldsymbol{n}_{\alpha, \alpha} d \Omega^{r} \\
& +\int_{\Gamma^{r}} \delta \boldsymbol{\theta} \cdot \boldsymbol{m}_{n} d \Gamma^{r}-\int_{\Omega^{r}} \delta \boldsymbol{\theta} \cdot \boldsymbol{m}_{\alpha, \alpha} d \Omega^{r} \\
& =-\int_{\Omega^{r}} \delta \boldsymbol{u} \cdot \boldsymbol{n}_{\alpha, \alpha} d \Omega^{r}+\int_{\Omega^{r}} \delta \boldsymbol{\theta} \cdot\left(\boldsymbol{Z}_{, \alpha}^{o} \boldsymbol{n}_{\alpha}-\boldsymbol{m}_{\alpha, \alpha}\right) d \Omega^{r} \\
& +\int_{\Gamma_{u}^{r}} \delta \boldsymbol{u} \cdot \boldsymbol{n}_{n} d \Gamma_{u}^{r}+\int_{\Gamma_{t}^{r}} \delta \boldsymbol{u} \cdot \boldsymbol{n}_{n} d \Gamma_{t}^{r}+\int_{\Gamma_{i}^{r}} \delta \boldsymbol{u} \cdot \boldsymbol{n}_{n} d \Gamma_{i}^{r} \\
& +\int_{\Gamma_{u}^{r}} \delta \boldsymbol{\theta} \cdot \boldsymbol{m}_{n} d \Gamma_{u}^{r}+\int_{\Gamma_{t}^{r}} \delta \boldsymbol{\theta} \cdot \boldsymbol{m}_{n} d \Gamma_{t}^{r}+\int_{\Gamma_{i}^{r}} \delta \boldsymbol{\theta} \cdot \boldsymbol{m}_{n} d \Gamma_{i}^{r} .
\end{aligned}
$$

We can then substitute this term into the full weak form (4.6) and gather the terms 
according to integration domain as to obtain

$$
\begin{aligned}
\delta W_{\text {lag }}= & \sum_{n_{r e g}}\left[-\int_{\Omega^{r}} \delta \boldsymbol{u} \cdot\left(\boldsymbol{n}_{\alpha, \alpha}+\overline{\boldsymbol{n}}^{\Omega^{r}}\right) d \Omega^{r}\right. \\
& \left.-\int_{\Omega^{r}} \delta \boldsymbol{\theta} \cdot\left(\boldsymbol{Z}_{, \alpha}^{o} \boldsymbol{n}_{\alpha}+\boldsymbol{m}_{\alpha, \alpha}+\overline{\boldsymbol{m}}^{\Omega^{r}}\right) d \Omega^{r}\right] \\
& +\int_{\Gamma_{t}^{r}} \delta \boldsymbol{u} \cdot\left(\boldsymbol{n}_{n}-\overline{\boldsymbol{n}}^{\Gamma_{t}^{r}}\right) d \Gamma_{t}^{r}+\int_{\Gamma_{t}^{r}} \delta \boldsymbol{\theta} \cdot\left(\boldsymbol{m}_{n}-\overline{\boldsymbol{m}}^{\Gamma_{t}^{r}}\right) d \Gamma_{t}^{r} \\
& +\int_{\Gamma_{u}^{r}} \delta \boldsymbol{u} \cdot\left(\boldsymbol{n}_{n}-\boldsymbol{n}^{\Gamma_{u}^{r}}\right) d \Gamma_{u}^{r}+\int_{\Gamma_{u}^{r}} \delta \boldsymbol{\theta} \cdot\left(\boldsymbol{m}_{n}-\boldsymbol{m}^{\Gamma_{u}^{r}}\right) d \Gamma_{u}^{r} \\
& -\int_{\Gamma_{u}^{r}} \delta \boldsymbol{n}^{\Gamma_{u}^{r}} \cdot[\boldsymbol{u}] d \Gamma_{u}^{r}-\int_{\Gamma_{u}^{r}} \delta \boldsymbol{m}^{\Gamma_{u}^{r}} \cdot[\boldsymbol{\theta}] d \Gamma_{u}^{r} \\
+ & \int_{\Gamma_{i}^{r}} \delta \boldsymbol{u}_{1} \cdot\left(\boldsymbol{n}_{1 n}-\boldsymbol{n}^{\Gamma_{i}^{r}}\right) d \Gamma_{i}^{r}+\int_{\Gamma_{i}^{r}} \delta \boldsymbol{u}_{2} \cdot\left(\boldsymbol{n}_{2 n}+\boldsymbol{n}^{\Gamma_{i}^{r}}\right) d \Gamma_{i}^{r} \\
+ & \int_{\Gamma_{i}^{r}} \delta \boldsymbol{\theta}_{1} \cdot\left(\boldsymbol{m}_{1 n}-\boldsymbol{m}^{\Gamma_{i}^{r}}\right) d \Gamma_{i}^{r}+\int_{\Gamma_{i}^{r}} \delta \boldsymbol{\theta}_{2} \cdot\left(\boldsymbol{m}_{2 n}+\boldsymbol{m}^{\Gamma_{i}^{r}}\right) d \Gamma_{i}^{r} \\
& -\int_{\Gamma_{i}^{r}} \delta \boldsymbol{n}^{\Gamma_{i}^{r}} \cdot[\boldsymbol{u}] d \Gamma_{i}^{r}-\int_{\Gamma_{i}^{r}} \delta \boldsymbol{m}^{\Gamma_{i}^{r}} \cdot[\boldsymbol{\theta}] d \Gamma_{i}^{r} \\
= & 0, \forall \delta \boldsymbol{u}, \delta \boldsymbol{\theta}, \delta \boldsymbol{n}^{\Gamma_{u}^{r}}, \delta \boldsymbol{m}^{\Gamma_{u}^{r}}, \delta \boldsymbol{n}^{\Gamma_{i}^{r}}, \delta \boldsymbol{m}^{\Gamma_{i}^{r}} .
\end{aligned}
$$

where $\boldsymbol{n}_{n}$ and $\boldsymbol{m}_{n}$ are the stresses normal to the boundary. Evoking the arbitrarity of the virtual fields, we can recover the strong formulation of our problem as

$$
\left.\begin{array}{r}
\boldsymbol{n}_{\alpha, \alpha}+\overline{\boldsymbol{n}}^{\Omega^{r}}=\boldsymbol{O} \\
\boldsymbol{m}_{\alpha, \alpha}+\boldsymbol{Z}_{, \alpha}^{o} \boldsymbol{n}_{\alpha}+\boldsymbol{m}_{\alpha, \alpha}=\boldsymbol{O}
\end{array}\right\} \text { in } \Omega^{r}
$$

that is the equilibrium statement for the domain,

$$
\left.\begin{array}{c}
\boldsymbol{n}_{n}=\overline{\boldsymbol{n}}^{\Gamma_{t}^{r}} \\
\boldsymbol{m}_{n}=\overline{\boldsymbol{m}}^{\Gamma_{t}^{r}}
\end{array}\right\} \text { on } \Gamma_{t}^{r}
$$

the equilibrium on the natural boundary between the tractions and the imposed external forces,

$$
\left.\begin{array}{r}
\boldsymbol{n}_{n}=\boldsymbol{n}^{\Gamma_{u}^{r}} \\
\boldsymbol{m}_{n}=\boldsymbol{m}^{\Gamma_{u}^{r}} \\
{[\boldsymbol{u}]=\boldsymbol{u}-\overline{\boldsymbol{u}}=\boldsymbol{O}} \\
{[\boldsymbol{\theta}]=\boldsymbol{\theta}-\overline{\boldsymbol{\theta}}=\boldsymbol{O}}
\end{array}\right\} \text { on } \Gamma_{u}^{r},
$$

which impose the equilibrium between the internal generalized stresses and the generalized reactions and the compatibility on the kinematic boundary, which are the independently approximated Lagrange Multipliers for the Dirichlet boundary. 


$$
\left.\begin{array}{r}
\boldsymbol{n}_{1 n}=-\boldsymbol{n}_{2 n}=\boldsymbol{n}^{\Gamma_{i}^{r}} \\
\boldsymbol{m}_{1 n}=-\boldsymbol{m}_{2 n}=\boldsymbol{m}_{i}^{\Gamma_{i}^{r}} \\
{[\boldsymbol{u}]=\boldsymbol{u}_{1}-\boldsymbol{u}_{2}=\boldsymbol{O}} \\
{[\boldsymbol{\theta}]=\boldsymbol{\theta}_{1}-\boldsymbol{\theta}_{2}=\boldsymbol{O}}
\end{array}\right\} \text { on } \Gamma_{i}^{r}
$$

states the equilibrium on the interface between the generalized stresses approximated from each region, identifies then with the Lagrange Multipliers for the interface and imposes continuity of generalized displacements across the interface.

The sets (4.14) - (4.17) are the Euler-Lagrange equations for the weak form presented.

\subsection{Penalty Method}

The prescribed displacements on the kinematic boundary and the continuity across the interfaces can also be imposed using the penalty method. This widely used and easily adaptable method have been used for many classes of problems. For the EFG method, a comparison with Lagrange Multipliers is available [60]. We penalize the error on the kinematic impositions with big enough constants $\beta_{u}$ and $\beta_{i}$. The added penalty potentials are

$$
\Pi_{p e n}^{u}=\frac{1}{2} \int_{\Gamma_{u}^{r}} \beta_{u}(\boldsymbol{d}-\overline{\boldsymbol{d}})^{2} d \Gamma_{u}^{r}
$$

for the Dirichlet boundary and

$$
\Pi_{p e n}^{i}=\frac{1}{2} \int_{\Gamma_{i}^{r}} \beta_{i}\left(\boldsymbol{d}_{1}-\boldsymbol{d}_{2}\right)^{2} d \Gamma_{i}^{r}
$$

for each interface. The variation of the previous potential yields the weak imposition, through penalty, of

$$
\begin{aligned}
\delta W_{p e n}^{u} & =\beta_{u} \int_{\Gamma_{u}^{r}} \delta \boldsymbol{d} \cdot(\boldsymbol{d}-\overline{\boldsymbol{d}}) d \Gamma_{u}^{r}=\beta_{u} \int_{\Gamma_{u}^{r}} \delta \boldsymbol{d} \cdot[\boldsymbol{d}] d \Gamma_{u}^{r} \\
& =0, \forall \delta \boldsymbol{d}
\end{aligned}
$$

the essential boundary conditions and

$$
\begin{aligned}
\delta W_{p e n}^{i} & =\beta_{i} \int_{\Gamma_{i}^{r}}\left(\delta \boldsymbol{d}_{1}-\delta \boldsymbol{d}_{2}\right) \cdot\left(\boldsymbol{d}_{1}-\boldsymbol{d}_{2}\right) d \Gamma_{i}^{r}=\beta_{i} \int_{\Gamma_{i}^{r}}[\delta \boldsymbol{d}] \cdot[\boldsymbol{d}] d \Gamma_{i}^{r} \\
& =0, \forall \delta \boldsymbol{d}_{1}, \delta \boldsymbol{d}_{2} .
\end{aligned}
$$

As this form is already symmetric, there is no need to add the complementary virtual 
works. The reaction forces and interface stresses are then given by

$$
\begin{aligned}
& \boldsymbol{q}^{\Gamma_{u}^{r}}=\beta_{u}(\boldsymbol{d}-\overline{\boldsymbol{d}}), \text { and } \\
& \boldsymbol{q}^{\Gamma_{i}^{r}}=\beta_{i}\left(\boldsymbol{d}_{1}-\boldsymbol{d}_{2}\right) .
\end{aligned}
$$

The choice of $\beta_{u}$ and $\beta_{i}$ is important as to guarantee numerical stability. The different order of magnitude between displacement and rotation DOFs and their energy conjugated stresses, along with their dependency on the shell thickness, suggests a split of the penalty between these quantities, so

$$
\begin{aligned}
\boldsymbol{n}^{\Gamma_{u}^{r}} & =\beta_{u}^{u}[\boldsymbol{u}], \boldsymbol{m}^{\Gamma_{u}^{r}}=\beta_{u}^{\theta}[\boldsymbol{\theta}] \\
\boldsymbol{n}^{\Gamma_{i}^{r}} & =\beta_{i}^{u}[\boldsymbol{u}], \boldsymbol{m}^{\Gamma_{i}^{r}}=\beta_{i}^{\theta}[\boldsymbol{\theta}]
\end{aligned}
$$

or

$$
\begin{aligned}
& \delta W_{\text {pen }}^{u}=\beta_{u}^{u} \int_{\Gamma_{u}^{r}} \delta \boldsymbol{u} \cdot[\boldsymbol{u}] d \Gamma_{u}^{r}+\beta_{u}^{\theta} \int_{\Gamma_{u}^{r}} \delta \boldsymbol{\theta} \cdot[\boldsymbol{\theta}] d \Gamma_{u}^{r}, \text { and } \\
& \delta W_{\text {pen }}^{i}=\beta_{i}^{u} \int_{\Gamma_{i}^{r}} \delta \boldsymbol{u} \cdot[\boldsymbol{u}] d \Gamma_{i}^{r}+\beta_{i}^{\theta} \int_{\Gamma_{i}^{r}} \delta \boldsymbol{\theta} \cdot[\boldsymbol{\theta}] d \Gamma_{i}^{r}
\end{aligned}
$$

We collectively refer to these penalty constants as $\beta_{u}$ and $\beta_{i}$ for the essential boundary and the interfaces respectively and as $\beta$ for the whole set. Both this equations must be introduced in (4.1) in order to obtain the final weak form for the penalty method.

$$
\begin{aligned}
\delta W_{\text {pen }} & =\sum_{n_{r e g}} \int_{\Omega^{r}} \delta \boldsymbol{\varepsilon}_{\alpha}^{r} \cdot \boldsymbol{\sigma}_{\alpha}^{r} d \Omega^{r}-\sum_{n_{r e g}} \int_{\Omega^{r}} \delta \boldsymbol{d} \cdot \overline{\boldsymbol{q}}^{\Omega^{r}} d \Omega^{r}-\int_{\Gamma_{t}^{r}} \delta \boldsymbol{d} \cdot \overline{\boldsymbol{q}}^{\Gamma_{t}^{r}} d \Gamma_{t}^{r} \\
& +\beta_{u}^{u} \int_{\Gamma_{u}^{r}} \delta \boldsymbol{u} \cdot[\boldsymbol{u}] d \Gamma_{u}^{r}+\beta_{u}^{\theta} \int_{\Gamma_{u}^{r}} \delta \boldsymbol{\theta} \cdot[\boldsymbol{\theta}] d \Gamma_{u}^{r} \\
& +\beta_{i}^{u} \int_{\Gamma_{i}^{r}}[\delta \boldsymbol{u}] \cdot[\boldsymbol{u}] d \Gamma_{i}^{r}+\beta_{i}^{\theta} \int_{\Gamma_{i}^{r}}[\delta \boldsymbol{\theta}] \cdot[\boldsymbol{\theta}] d \Gamma_{i}^{r} \\
& =0, \forall \delta \boldsymbol{d}
\end{aligned}
$$

It's important to note that modifying the weak form with penalty terms don't introduce new unknowns to the problem or require any new integration scheme, although, results are highly dependent on the constants $\beta$. Actually, the original differential equations cannot be recovered from the modified weak form, as the addition of the penalty terms in fact modify the strong formulation.

We can extract the Euler-Lagrange equations by substituting the last expression for $\delta W_{\text {int }}$ in (4.12) into (4.25). 


$$
\begin{aligned}
\delta W_{\text {pen }} & =\sum_{n_{\text {reg }}}\left[-\int_{\Omega^{r}} \delta \boldsymbol{u} \cdot\left(\boldsymbol{n}_{\alpha, \alpha}+\overline{\boldsymbol{n}}^{\Omega^{r}}\right) d \Omega^{r}\right. \\
& \left.-\int_{\Omega^{r}} \delta \boldsymbol{\theta} \cdot\left(\boldsymbol{Z}_{, \alpha}^{o} \boldsymbol{n}_{\alpha}+\boldsymbol{m}_{\alpha, \alpha}+\overline{\boldsymbol{m}}^{\Omega^{r}}\right) d \Omega^{r}\right] \\
& +\int_{\Gamma_{t}^{r}} \delta \boldsymbol{u} \cdot\left(\boldsymbol{n}_{n}-\overline{\boldsymbol{n}}^{\Gamma_{t}^{r}}\right) d \Gamma_{t}^{r}+\int_{\Gamma_{t}^{r}} \delta \boldsymbol{\theta} \cdot\left(\boldsymbol{m}_{n}-\overline{\boldsymbol{m}}^{\Gamma_{t}^{r}}\right) d \Gamma_{t}^{r} \\
& +\int_{\Gamma_{u}^{r}} \delta \boldsymbol{u} \cdot\left(\boldsymbol{n}_{n}+\beta_{u}^{u}[\boldsymbol{u}]\right) d \Gamma_{u}^{r}+\int_{\Gamma_{u}^{r}} \delta \boldsymbol{\theta} \cdot\left(\boldsymbol{m}_{n}+\beta_{u}^{\theta}[\boldsymbol{\theta}]\right) d \Gamma_{u}^{r} \\
& +\int_{\Gamma_{i}^{r}} \delta \boldsymbol{u}_{1} \cdot\left(\boldsymbol{n}_{1 n}+\beta_{i}^{u}[\boldsymbol{u}]\right) d \Gamma_{i}^{r}+\int_{\Gamma_{i}^{r}} \delta \boldsymbol{u}_{2} \cdot\left(\boldsymbol{n}_{2 n}-\beta_{i}^{u}[\boldsymbol{u}]\right) d \Gamma_{i}^{r} \\
& +\int_{\Gamma_{i}^{r}} \delta \boldsymbol{\theta}_{1} \cdot\left(\boldsymbol{m}_{1 n}+\beta_{i}^{\theta}[\boldsymbol{\theta}]\right) d \Gamma_{i}^{r}+\int_{\Gamma_{i}^{r}} \delta \boldsymbol{\theta}_{2} \cdot\left(\boldsymbol{m}_{2 n}-\beta_{i}^{\theta}[\boldsymbol{\theta}]\right) d \Gamma_{i}^{r} \\
= & 0, \forall \delta \boldsymbol{u}^{1}, \delta \boldsymbol{u}^{2}, \delta \boldsymbol{\theta}^{1}, \delta \boldsymbol{\theta}^{2}
\end{aligned}
$$

and accounting for the arbitrarity of $\delta \boldsymbol{d}$, the strong form related to the penalty weak form is

$$
\left.\begin{array}{c}
\boldsymbol{n}_{\alpha, \alpha}+\overline{\boldsymbol{n}}^{\Omega^{r}}=\boldsymbol{O} \\
\boldsymbol{m}_{\alpha, \alpha}+\boldsymbol{Z}_{, \alpha}^{o} \boldsymbol{n}_{\alpha}+\boldsymbol{m}_{\alpha, \alpha}=\boldsymbol{O}
\end{array}\right\} \text { in } \Omega^{r},
$$

as (4.14) and (4.28) for the equilibrium in the domain and on the natural boundary,

$$
\left.\begin{array}{l}
\boldsymbol{n}_{n}=-\beta_{u}^{u}[\boldsymbol{u}] \\
\boldsymbol{m}_{n}=-\beta_{u}^{\theta}[\boldsymbol{\theta}]
\end{array}\right\} \text { on } \Gamma_{u}^{r},
$$

on the kinematic boundary, and

$$
\left.\begin{array}{r}
\boldsymbol{n}_{n}^{1}=-\boldsymbol{n}_{n}^{2}=-\beta_{i}^{u}[\boldsymbol{u}] \\
\boldsymbol{m}_{n}^{1}=-\boldsymbol{m}_{n}^{2}=-\beta_{i}^{\theta}[\boldsymbol{\theta}]
\end{array}\right\} \text { on } \Gamma_{i}^{r}
$$

on the interfaces.

It can be noticed that the domain and natural boundary equilibrium is recovered, but kinematic constraints are not exactly enforced. Eq. 4.29 shows a dependency between the normal tractions on the kinematic boundary and the domain displacements that makes the coherent imposition only as $\beta_{u}$ tends to infinite.

Similarly on the interface equilibrium is kept only if the gap between approximations is not null or if the constant assumes a very large value. Numerically, this is impossible, as large values for $\beta$ affects the condition of the discrete system of equations and consequently 
the quality of the solution.

Nevertheless, the Penalty method is easily adaptable for many classes of problems and easily implemented in computer codes. The discretized version of (4.25) with nodalbased functions as in (3.9) and (3.12) is a linear system of equations of the size of $\mathbf{d}$, the approximation field for the primary variables.

$$
\left[\mathbf{K}+\mathbf{P}_{p e n}^{u}+\mathbf{P}_{p e n}^{i}\right] \mathbf{d}=\mathbf{f}^{\Omega^{r}}+\mathbf{f}^{\Gamma_{t}^{r}}+\mathbf{q}_{p e n}^{u}
$$

Some observations must be made as to how the degrees of freedom are organized in (4.31). If we assume

$$
\mathbf{d}=\left[\begin{array}{llll}
\mathbf{u}_{1}^{T} & \boldsymbol{\theta}_{1}^{T} & \mathbf{u}_{2}^{T} & \boldsymbol{\theta}_{2}^{T}
\end{array}\right]^{T}
$$

where $\mathbf{u}_{n}$ and $\boldsymbol{\theta}_{n}$ respectively gather the degrees of freedom used to describe the displacements and rotations in region $n$, we have

$$
\mathbf{K}=\left[\begin{array}{cc}
\mathbf{K}_{1} & \mathbf{0} \\
\mathbf{0} & \mathbf{K}_{2}
\end{array}\right]
$$

for the global stiffness matrix, with each region's stiffness $\mathbf{K}_{n}$ given by Eq. (4.8)),

$$
\mathbf{P}_{p e n}^{u}=\left[\begin{array}{cccc}
\beta_{u}^{u} \mathbf{P}_{p e n}^{u u} & \mathbf{0} & \mathbf{0} & \mathbf{0} \\
\mathbf{0} & \beta_{u}^{\theta} \mathbf{P}_{p e n}^{u \theta} & \mathbf{0} & \mathbf{0} \\
\mathbf{0} & \mathbf{0} & \mathbf{0} & \mathbf{0} \\
\mathbf{0} & \mathbf{0} & \mathbf{0} & \mathbf{0}
\end{array}\right]
$$

for the penalty matrix on the essential boundary. The penalty matrix must be separated by type of DOF because, as stated before, we use different penalty parameters for displacements and rotations. These matrices are respectively

$$
\begin{aligned}
& \mathbf{P}_{p e n}^{u u}=\int_{\Gamma_{u}^{r}} \boldsymbol{\Phi}^{u T} \boldsymbol{\Phi}^{u} d \Gamma_{u}^{r} \\
& \mathbf{P}_{p e n}^{u \theta}=\int_{\Gamma_{u}^{r}} \boldsymbol{\Phi}^{\theta T} \boldsymbol{\Phi}^{\theta} d \Gamma_{u}^{r}
\end{aligned}
$$

For the interface penalty constraints, assuming the same arrangement and separation of the DOFs, the penalty matrix is

$$
\mathbf{P}_{p e n}^{i}=\left[\begin{array}{cccc}
\beta_{i}^{u} \mathbf{P}_{11}^{i u} & \mathbf{0} & \beta_{i}^{u} \mathbf{P}_{12}^{i u} & \mathbf{0} \\
\mathbf{0} & \beta_{i}^{\theta} \mathbf{P}_{11}^{i \theta} & \mathbf{0} & \beta_{i}^{\theta} \mathbf{P}_{12}^{i \theta} \\
\beta_{i}^{u} \mathbf{P}_{21}^{i u} & \mathbf{0} & \beta_{i}^{u} \mathbf{P}_{22}^{i u} & \mathbf{0} \\
\mathbf{0} & \beta_{i}^{\theta} \mathbf{P}_{21}^{i \theta} & \mathbf{0} & \beta_{i}^{\theta} \mathbf{P}_{22}^{i \theta}
\end{array}\right]
$$


with each of the submatrices given by

$$
\begin{gathered}
\mathbf{P}_{a b}^{i u}=\left(2 \delta_{a b}-1\right) \int_{\Gamma_{i}^{r}} \boldsymbol{\Phi}_{a}^{u T} \boldsymbol{\Phi}_{b}^{u} d \Gamma_{i}^{r}, \\
\mathbf{P}_{a b}^{i \theta}=\left(2 \delta_{a b}-1\right) \int_{\Gamma_{i}^{r}} \boldsymbol{\Phi}_{a}^{\theta T} \boldsymbol{\Phi}_{b}^{\theta} d \Gamma_{i}^{r}, \\
(a, b) \in[1,2]
\end{gathered}
$$

and $\boldsymbol{\Phi}_{i}^{u}$ and $\boldsymbol{\Phi}_{i}^{\theta}$ are the shape functions used to approximate respectively the displacements and rotations in the domain $\Omega_{i}^{r}$. The force vectors $\mathbf{f}^{\Omega^{r}}$ and $\mathbf{f}^{\Gamma_{t}^{r}}$ are the same as in (4.11), and the essential boundary equivalent force is

$$
\mathbf{q}_{p e n}^{u}=\left[\begin{array}{l}
\int_{\Gamma_{u}^{r}} \beta_{u}^{u} \Phi^{u T} \overline{\boldsymbol{u}} d \Gamma_{u}^{r} \\
\int_{\Gamma_{u}^{r}} \beta_{u}^{\theta} \boldsymbol{\Phi}^{\theta T} \overline{\boldsymbol{\theta}} d \Gamma_{u}^{r}
\end{array}\right] .
$$

\subsection{Nitsche's Method}

The interior penalty method described by Nitsche [47] uses the internal variable to describe the flux across the essential boundary. In this model problem, the internal fields are the displacements and rotations gathered in $\boldsymbol{d}$ and used to describe the shell stresses in the Dirichlet boundary $\boldsymbol{\sigma}_{n}^{r}$, which by their turn will be equal to the reaction forces. Similar approaches have been used by Fernández-Méndez and Huerta [18] for imposing EBCs in a meshless approximation to solve a uniform Poisson's problem and by Nguyen et. al. [46] for 2-D and 3-D elasticity in the context of isogeometric analysis.

In our shell problem, we state that

$$
\boldsymbol{r}^{r}=\left.\boldsymbol{P} \boldsymbol{n}^{r}\right|_{\Gamma_{u}^{r}}
$$

In (4.40), $\boldsymbol{r}^{r}$ is the normal reaction force back-rotated to the reference configuration, $\boldsymbol{P}$ is the first Piolla-Kirchhoff stress tensor and $\boldsymbol{n}^{r}$ is the unit outward normal to the essential boundary at the reference configuration. The integration of $\boldsymbol{r}^{r}$ through the thickness yields the generalized reaction resultants of $(2.35 \mathrm{c})$, which according to the method, are identified with the tractions on the boundary given by the domain generalized displacement approximation.

$$
\boldsymbol{\sigma}^{\Gamma_{u}^{r}}=\boldsymbol{\sigma}_{n}^{r}=n_{\alpha}^{r} \boldsymbol{\sigma}_{\alpha}^{r}(\boldsymbol{d}),
$$

where $n_{\alpha}^{r}$ are the components of $\boldsymbol{n}^{r}$ in $\boldsymbol{e}_{\alpha}^{r}$ as this back-rotated normal has no component perpendicular to the reference plane $\left(\boldsymbol{n}^{r} \cdot \boldsymbol{e}_{3}^{r}=0\right)$. 
The weak imposition of the displacements takes a form similar to (4.3):

$$
\delta W_{n i t}^{u}=\int_{\Gamma_{u}^{r}} \delta \boldsymbol{\sigma}_{n}^{r} \cdot[\boldsymbol{d}] d \Gamma_{u}^{r}=0, \forall \delta \boldsymbol{\sigma}_{n}^{r}
$$

but now the generalized reaction forces are not independently approximated, but derived from the domain displacements.

Using (2.45) and (2.42), one can define the back-rotated stress in a given direction $\boldsymbol{n}^{r}=n_{\alpha}^{r} \boldsymbol{e}_{\alpha}^{r}$ in the reference configuration, due to a set of generalized displacements $\boldsymbol{d}$ as

$$
\boldsymbol{\sigma}_{n}^{r}(\boldsymbol{d})=n_{\alpha}^{r} \boldsymbol{\sigma}_{\alpha}^{r}=n_{\alpha}^{r} \boldsymbol{D}_{\alpha \beta} \boldsymbol{\Delta}_{\beta} \boldsymbol{d}
$$

so that the complementary virtual work of the boundary reactions is

$$
\delta W_{n i t}^{u}=n_{\alpha}^{r} \int_{\Gamma_{u}^{r}} \boldsymbol{\sigma}_{\alpha}^{r}(\delta \boldsymbol{d}) \cdot[\boldsymbol{d}] d \Gamma_{u}^{r}=0, \forall \delta \boldsymbol{d} .
$$

Nitsche's weak form comprised also a penalty-like stabilization parameter as to maintain the coercivity of the bilinear form, or the positive definite characteristic of the discretized problem. So Eq. 4.44 can be adapted to

$$
\delta W_{n i t}^{u}=\int_{\Gamma_{u}^{r}}\left(\boldsymbol{\sigma}_{n}^{r}(\delta \boldsymbol{d})-\beta_{u} \delta \boldsymbol{d}\right) \cdot[\boldsymbol{d}] d \Gamma_{u}^{r}=0, \forall \delta \boldsymbol{d} .
$$

In the same fashion as done for the Penalty Method in (4.23a), we divide the impositions into membrane and flexural stresses as to split their separate contributions to the stability of the problem. The split imposition is then

$$
\begin{aligned}
\delta W_{n i t}^{u} & =\int_{\Gamma_{u}^{r}}\left(\boldsymbol{n}_{n}^{r}(\delta \boldsymbol{d})-\beta_{u}^{u} \delta \boldsymbol{u}\right) \cdot[\boldsymbol{u}] d \Gamma_{u}^{r} \\
& +\int_{\Gamma_{u}^{r}}\left(\boldsymbol{m}_{n}^{r}(\delta \boldsymbol{d})-\beta_{u}^{\theta} \delta \boldsymbol{\theta}\right) \cdot[\boldsymbol{\theta}] d \Gamma_{u}^{r}
\end{aligned}
$$

For the interface conditions, the tractions are taken any convex average of the stresses from the intersecting regions

$$
\left\langle\boldsymbol{\sigma}_{n}^{r}\right\rangle=\gamma \boldsymbol{\sigma}_{n}^{r}\left(\boldsymbol{d}_{1}\right)+(1-\gamma) \boldsymbol{\sigma}_{n}^{r}\left(\boldsymbol{d}_{2}\right)
$$

The weight $\gamma$ can be any value between zero and unity $[53,1]$. We make the trivial choice of $\gamma=0.5$.

The interface constrains are then imposed in as similar manner to the EBCs via the weak statement

$$
\delta W_{n i t}^{i}=\int_{\Gamma_{i}^{r}}\left(\left\langle\delta \boldsymbol{\sigma}_{n}^{r}\right\rangle-\beta_{i}[\delta \boldsymbol{d}]\right) \cdot[\boldsymbol{d}] d \Gamma_{i}^{r}+\int_{\Gamma_{i}^{r}}\left\langle\boldsymbol{\sigma}_{n}^{r}\right\rangle \cdot[\delta \boldsymbol{d}] d \Gamma_{i}^{r} .
$$


Once again, we divide the imposition between in-plane and out-of-plane stresses as the behavior of the different types of DOFs is highly dependent on the thickness of the shell. Similar approach was carried out in [49], where the impositions for normal and shear stresses were separated and the stabilization parameters for each kind of stress was independently determined. The expanded weak contribution is then

$$
\begin{aligned}
\delta W_{n i t}^{i} & =\int_{\Gamma_{i}^{r}}\left(\left\langle\delta \boldsymbol{n}_{n}^{r}\right\rangle-\beta_{i}^{u}[\delta \boldsymbol{u}]\right) \cdot[\boldsymbol{u}] d \Gamma_{i}^{r}+\int_{\Gamma_{i}^{r}}\left(\left\langle\delta \boldsymbol{m}_{n}^{r}\right\rangle-\beta_{i}^{\theta}[\delta \boldsymbol{\theta}]\right) \cdot[\boldsymbol{\theta}] d \Gamma_{i}^{r} \\
& +\int_{\Gamma_{i}^{r}}\left\langle\boldsymbol{n}_{n}^{r}\right\rangle \cdot[\delta \boldsymbol{u}] d \Gamma_{i}^{r}+\int_{\Gamma_{i}^{r}}\left\langle\boldsymbol{m}_{n}^{r}\right\rangle \cdot[\delta \boldsymbol{\theta}] d \Gamma_{i}^{r}
\end{aligned}
$$

The global weak form for the Nitsche's Method is then

$$
\begin{aligned}
\delta W_{n i t}= & \sum_{n_{r e g}} \int_{\Omega^{r}} \delta \boldsymbol{\varepsilon}_{\alpha}^{r} \cdot \boldsymbol{\sigma}_{\alpha}^{r} d \Omega^{r}-\sum_{n_{r e g}} \int_{\Omega^{r}} \delta \boldsymbol{d} \cdot \overline{\boldsymbol{q}}^{\Omega^{r}} d \Omega^{r}-\int_{\Gamma_{t}^{r}} \delta \boldsymbol{d} \cdot \overline{\boldsymbol{q}}^{\Gamma_{t}^{r}} d \Gamma_{t}^{r} \\
& -\int_{\Gamma_{u}^{r}} \delta \boldsymbol{u} \cdot \boldsymbol{n}_{n}^{r} d \Gamma_{u}^{r}-\int_{\Gamma_{u}^{r}} \delta \boldsymbol{n}_{n}^{r} \cdot[\boldsymbol{u}] d \Gamma_{u}^{r}+\beta_{u}^{u} \int_{\Gamma_{u}^{r}} \delta \boldsymbol{u} \cdot[\boldsymbol{u}] d \Gamma_{u}^{r} \\
& -\int_{\Gamma_{u}^{r}} \delta \boldsymbol{\theta} \cdot \boldsymbol{m}_{n}^{r} d \Gamma_{u}^{r}-\int_{\Gamma_{u}^{r}} \delta \boldsymbol{m}_{n}^{r} \cdot[\boldsymbol{\theta}] d \Gamma_{u}^{r}+\beta_{u}^{\theta} \int_{\Gamma_{u}^{r}} \delta \boldsymbol{\theta} \cdot[\boldsymbol{\theta}] d \Gamma_{u}^{r} \\
& -\int_{\Gamma_{i}^{r}}\left\langle\delta \boldsymbol{n}_{n}^{r}\right\rangle \cdot[\boldsymbol{u}] d \Gamma_{i}^{r}-\int_{\Gamma_{i}^{r}}\left\langle\boldsymbol{n}_{n}^{r}\right\rangle \cdot[\delta \boldsymbol{u}] d \Gamma_{i}^{r}+\beta_{i}^{u} \int_{\Gamma_{i}^{r}}[\delta \boldsymbol{u}] \cdot[\boldsymbol{u}] d \Gamma_{i}^{r} \\
& -\int_{\Gamma_{i}^{r}}\left\langle\delta \boldsymbol{m}_{n}^{r}\right\rangle \cdot[\boldsymbol{\theta}] d \Gamma_{i}^{r}-\int_{\Gamma_{i}^{r}}\left\langle\boldsymbol{m}_{n}^{r}\right\rangle \cdot[\delta \boldsymbol{\theta}] d \Gamma_{i}^{r}+\beta_{i}^{\theta} \int_{\Gamma_{i}^{r}} \delta[\boldsymbol{\theta}] \cdot[\boldsymbol{\theta}] d \Gamma_{i}^{r}, \\
= & 0, \forall \delta \boldsymbol{u}, \delta \boldsymbol{\theta}
\end{aligned}
$$

where $\boldsymbol{n}_{n}^{r}, \boldsymbol{m}_{n}^{r}$ and their variations are expressed as linearly dependents on the displacements $\boldsymbol{d}$. As has been pointed out by Tiago [58], Nitsche's weak form is a combination between the modified variational principle early developed [42] and a penalty enforcement. It can also be derived from an Augmented Lagrangian formulation where the Lagrange Multipliers are replaced by the boundary tractions.

After discretization of both trial and test spaces of generalized displacements with the same shape functions, and taking into account the general characteristic of the variations, the linear system of equations is obtained as

$$
\left[\mathbf{K}+\mathbf{G}_{n i t}^{u}+\mathbf{P}_{n i t}^{u}+\mathbf{G}_{n i t}^{i}+\mathbf{P}_{n i t}^{i}\right] \mathbf{d}=\mathbf{f}^{\Omega^{r}}+\mathbf{f}^{\Gamma_{t}^{r}}+\mathbf{q}_{n i t}^{u}
$$

The stiffness matrix $\mathbf{K}$ is defined by Eq. (4.33) and the force vectors $\mathbf{f}^{\Omega^{r}}$ and $\mathbf{f}^{\Gamma_{t}^{r}}$ are the given by (4.11). The penalty matrices $\mathbf{P}_{n i t}^{u}$ and $\mathbf{P}_{n i t}^{i}$ are the same as for the penalty method $\mathbf{P}_{\text {pen }}^{u}$ and $\mathbf{P}_{\text {pen }}^{i}$ given by Eq. (4.34) and Eq. (4.37) respectively. The (symmetric) 
boundary imposition matrix is given by

$$
\mathbf{G}_{n i t}^{u}=-n_{\alpha}^{r} \int_{\Gamma_{u}^{r}} \boldsymbol{\Phi}^{T} \mathbf{D}_{\alpha \beta} \mathbf{B}_{\beta} d \Gamma_{u}^{r}-n_{\alpha}^{r} \int_{\Gamma_{u}^{r}} \mathbf{B}_{\beta}^{T} \mathbf{D}_{\alpha \beta}^{T} \boldsymbol{\Phi} d \Gamma_{u}^{r}
$$

For the interfaces, some assembly is required, similarly to the penalty matrices,

$$
\mathbf{G}_{n i t}^{i}=\frac{1}{2}\left[\begin{array}{ll}
\mathbf{G}_{11}^{i} & \mathbf{G}_{12}^{i} \\
\mathbf{G}_{21}^{i} & \mathbf{G}_{22}^{i}
\end{array}\right]+\frac{1}{2}\left[\begin{array}{cc}
\mathbf{G}_{11}^{i} & \mathbf{G}_{12}^{i} \\
\mathbf{G}_{21}^{i} & \mathbf{G}_{22}^{i}
\end{array}\right]^{T}
$$

whose component matrices are

$$
\begin{array}{r}
\mathbf{G}_{a b}^{i}=\left(2 \delta_{a b}-1\right) \int_{\Gamma_{i}^{r}} n_{\alpha}^{r} \boldsymbol{\Phi}^{a T} \mathbf{D}_{\alpha \beta}^{b} \mathbf{B}_{\beta}^{b} d \Gamma_{i}^{r}, \\
a, b \in[1,2] .
\end{array}
$$

Vector $\mathbf{q}_{n i t}^{u}$ accounts for the imposed generalized displacements. It involves both the penalty imposition vector $\mathbf{q}_{p e n}^{u}(4.39)$ and an extra contribution

$$
\mathbf{q}_{n i t}^{u}=\mathbf{q}_{p e n}^{u}-\int_{\Gamma_{u}^{r}} n_{\alpha}^{r} \mathbf{D}_{\alpha \beta} \mathbf{B}_{\beta} \overline{\boldsymbol{d}} d \Gamma_{u}^{r}
$$

from Nitsche's terms.

Contrary to the Penalty Method, the constants $\beta$ do not play a much significant role in the behavior of the system. The strong form can be recovered independently of the choice of constants. We use the notation for the tractions calculated from the domain approximation as

$$
\begin{aligned}
\boldsymbol{n}_{1 n} & =n_{1 \alpha} \boldsymbol{n}_{1}=n_{1 \alpha} \boldsymbol{D}_{\alpha \beta}^{\eta} \boldsymbol{\Delta}_{\beta}^{\eta} \boldsymbol{d}_{1} \\
\boldsymbol{n}_{2 n} & =n_{2 \alpha} \boldsymbol{n}_{2}=n_{2 \alpha} \boldsymbol{D}_{\alpha \beta}^{\eta} \boldsymbol{\Delta}_{\beta}^{\eta} \boldsymbol{d}_{2} \\
\boldsymbol{m}_{1 n} & =n_{1 \alpha} \boldsymbol{m}_{1}=n_{1 \alpha} \boldsymbol{D}_{\alpha \beta}^{\kappa} \boldsymbol{\Delta}_{\beta}^{\kappa} \boldsymbol{d}_{1} \\
\boldsymbol{m}_{2 n} & =n_{2 \alpha} \boldsymbol{m}_{2}=n_{2 \alpha} \boldsymbol{D}_{\alpha \beta}^{\kappa} \boldsymbol{\Delta}_{\beta}^{\kappa} \boldsymbol{d}_{2},
\end{aligned}
$$

where $n_{1 \alpha}$ and $n_{2 \alpha}$ are the components for the normal vector of each region. We draw the attention to the fact that, in this case, the normals are not opposing $\left(n_{1 \alpha} \neq-n_{2 \alpha}\right)$ as the 
interface might be a kink where the midsurface changes plane. From (4.50), one can write

$$
\begin{aligned}
\delta W_{n i t} & =\sum_{n_{r e g}}\left[-\int_{\Omega^{r}} \delta \boldsymbol{u} \cdot\left(\boldsymbol{n}_{\alpha, \alpha}+\overline{\boldsymbol{n}}^{\Omega^{r}}\right) d \Omega^{r}\right. \\
& \left.-\int_{\Omega^{r}} \delta \boldsymbol{\theta} \cdot\left(\boldsymbol{Z}_{, \alpha}^{o} \boldsymbol{n}_{\alpha}+\boldsymbol{m}_{\alpha, \alpha}+\overline{\boldsymbol{m}}^{\Omega^{r}}\right) d \Omega^{r}\right] \\
& +\int_{\Gamma_{t}^{r}} \delta \boldsymbol{u} \cdot\left(\boldsymbol{n}_{n}-\overline{\boldsymbol{n}}_{t}^{\Gamma_{t}^{r}}\right) d \Gamma_{t}^{r}+\int_{\Gamma_{t}^{r}} \delta \boldsymbol{\theta} \cdot\left(\boldsymbol{m}_{n}-\overline{\boldsymbol{m}}^{\Gamma_{t}^{r}}\right) d \Gamma_{t}^{r} \\
& +\int_{\Gamma_{u}^{r}} \delta \boldsymbol{u} \cdot\left(\boldsymbol{n}_{n}-\boldsymbol{n}_{1 n}\right) d \Gamma_{u}^{r}-\int_{\Gamma_{u}^{r}}\left(\delta \boldsymbol{n}_{1 n}-\beta_{u}^{u} \delta \boldsymbol{u}\right) \cdot[\boldsymbol{u}] d \Gamma_{u}^{r} \\
& +\int_{\Gamma_{u}^{r}} \delta \boldsymbol{\theta} \cdot\left(\boldsymbol{m}_{n}-\boldsymbol{m}_{1 n}\right) d \Gamma_{u}^{r}-\int_{\Gamma_{u}^{r}}\left(\delta \boldsymbol{m}_{1 n}-\beta_{u}^{\theta} \delta \boldsymbol{\theta}\right) \cdot[\boldsymbol{\theta}] d \Gamma_{u}^{r} \\
& +\int_{\Gamma_{i}^{r}} \delta \boldsymbol{u}_{1} \cdot\left(\boldsymbol{n}_{1 n}-\left\langle\boldsymbol{n}_{n}\right\rangle\right) d \Gamma_{i}^{r}+\int_{\Gamma_{i}^{r}} \delta \boldsymbol{u}_{2} \cdot\left(\boldsymbol{n}_{2 n}+\left\langle\boldsymbol{n}_{n}\right\rangle\right) d \Gamma_{i}^{r} \\
& +\int_{\Gamma_{i}^{r}} \delta \boldsymbol{\theta}_{1} \cdot\left(\boldsymbol{m}_{1 n}-\left\langle\boldsymbol{m}_{n}\right\rangle\right) d \Gamma_{i}^{r}+\int_{\Gamma_{i}^{r}} \delta \boldsymbol{\theta}_{2} \cdot\left(\boldsymbol{m}_{2 n}+\left\langle\boldsymbol{m}_{n}\right\rangle\right) d \Gamma_{i}^{r} \\
& -\int_{\Gamma_{i}^{r}}\left(\left\langle\delta \boldsymbol{n}_{n}^{r}\right\rangle-\beta_{i}^{u}[\delta \boldsymbol{u}]\right) \cdot[\boldsymbol{u}] d \Gamma_{i}^{r} \\
& -\int_{\Gamma_{i}^{r}}\left(\left\langle\delta \boldsymbol{m}_{n}^{r}\right\rangle-\beta_{i}^{\theta}[\delta \boldsymbol{\theta}]\right) \cdot[\boldsymbol{\theta}] d \Gamma_{i}^{r} \\
= & 0, \forall \delta \boldsymbol{u}, \delta \boldsymbol{\theta}
\end{aligned}
$$

A careful choice of the virtual fields can present the strong formulation for the shell. Equilibrium in the domain comes from the first two terms as

$$
\left.\begin{array}{r}
\boldsymbol{n}_{\alpha, \alpha}+\overline{\boldsymbol{n}}^{\Omega^{r}}=\boldsymbol{O} \\
\boldsymbol{m}_{\alpha, \alpha}+\boldsymbol{Z}_{, \alpha}^{o} \boldsymbol{n}_{\alpha}+\boldsymbol{m}_{\alpha, \alpha}=\boldsymbol{O}
\end{array}\right\} \text { in } \Omega^{r}
$$

Appropriate election on the natural boundary provide

$$
\left.\begin{array}{rl}
\boldsymbol{n}_{n} & =\overline{\boldsymbol{n}}^{\Gamma_{t}^{r}} \\
\boldsymbol{m}_{n} & =\overline{\boldsymbol{m}}^{\Gamma_{t}^{r}}
\end{array}\right\} \text { on } \Gamma_{t}^{r},
$$

the equilibrium on $\Gamma_{t}^{r}$. For the essential boundary we can obtain

$$
\left.\begin{array}{r}
\text { if } \delta \boldsymbol{u} \neq \boldsymbol{O}, \boldsymbol{n}_{n}=\boldsymbol{n}_{1 n} \\
\text { if } \delta \boldsymbol{\theta} \neq \boldsymbol{O}, \boldsymbol{m}_{n}=\boldsymbol{m}_{1 n} \\
\text { if } \delta \boldsymbol{n}_{1 n}-\beta_{u}^{u} \delta \boldsymbol{u} \neq \boldsymbol{O},[\boldsymbol{u}]=\boldsymbol{u}-\overline{\boldsymbol{u}}=\boldsymbol{O} \\
\text { if } \delta \boldsymbol{m}_{1 n}-\beta_{u}^{u} \delta \boldsymbol{u} \neq \boldsymbol{O},[\boldsymbol{\theta}]=\boldsymbol{\theta}-\overline{\boldsymbol{\theta}}=\boldsymbol{O}
\end{array}\right\} \text { on } \Gamma_{u}^{r},
$$

the identification of the internal generalized stresses normal to the boundary with the generalized reactions and the compatibility of displacements and rotations. If similar 
assumptions are made on the interface,

$$
\left.\begin{array}{r}
\boldsymbol{n}_{1 n}=-\boldsymbol{n}_{2 n}=\left\langle\boldsymbol{n}_{n}\right\rangle \\
\boldsymbol{m}_{1 n}=-\boldsymbol{m}_{2 n}=\left\langle\boldsymbol{m}_{n}\right\rangle \\
{[\boldsymbol{u}]=\boldsymbol{u}_{1}-\boldsymbol{u}_{2}=\boldsymbol{O}} \\
{[\boldsymbol{\theta}]=\boldsymbol{\theta}_{1}-\boldsymbol{\theta}_{2}=\boldsymbol{O}}
\end{array}\right\} \text { on } \Gamma_{i}^{r} .
$$

Eq. 4.61 expresses that the tractions on both regions at a point on the interface are in balance and that the relative displacements and rotations are compatible.

An important issue with Nitsche's weak formulation is the determination of constants $\beta$. Even though results are not so sensitive to this parameter as in a penalty framework, a lower bound is necessary to keep the bilinear form coercivity and a upper bound to refrain from ill-conditioning the system.

Some works ([46]) choose $\beta$ empirically from the order of magnitude of the other matrices involved. [52] presents a global eigenproblem whose solution yields a good estimate for the parameter in an eXtended Finite Element (XFEM) framework, as do [20] for imposing EBCs for a Partition of Unity meshless method. This technique is cited by [18], but the parameter is obtained from a "parametric tuning". A similar problem is assembled element by element in [16] for B-splines-based Finite Elements. Those eigenproblems are smaller than a global one for compactly support shape functions, but the stabilization parameter varies along the boundary. [15, 1] reach analytical expressions for the parameters for triangular and tetrahedral finite elements, and [53] uses the largest of these (and others) element wide estimators as a global constant.

An interesting approach is taken by [49] for enforcing EBCs, where the normal and tangential stress components are taken separately and have different parameters defined in two separate local eigenproblems. Similar approach is taken in [50] for isogeometric analysis of patched geometries, where the local eigenproblem is used to estimate the stabilization over the interfaces.

If the local approach was to be adapted for a meshless framework, local eigenproblems would have to be assembled for each integration point, as their connectivity are unique and determined during the computation of the shape functions. It can be said that, although compact, MLS shape functions support are larger than those of FEs and the connectivity is more populated, especially for higher order polynomial bases. Since the size of the local eigenvalue problem is related to this connectivity, it wouldn't be much smaller than a global one when MLS shape functions are used, and we would have to solve many of these eigenvalue problems. For that reason, and for local instabilities that can arise in a local approach, this work makes use of global eigenproblems to determine the stabilization parameters.

We start by expressing the virtual works as linear and bilinear forms. The internal 
virtual work translates into a bilinear form

$$
a^{i n t}(\delta \boldsymbol{d}, \boldsymbol{d})=\delta W^{i n t}=\int_{\Omega^{r}} \delta \varepsilon^{e r} \cdot \boldsymbol{\sigma}^{r} d \Omega^{r} .
$$

and the external virtual work into a linear form

$$
l^{e x t}(\delta \boldsymbol{d})=-\delta W^{e x t}=-\int_{\Omega^{r}} \delta \boldsymbol{d} \cdot \overline{\boldsymbol{q}}^{\Omega^{r}} d \Omega^{r}-\int_{\Gamma_{t}^{r}} \delta \boldsymbol{d} \cdot \overline{\boldsymbol{q}}^{\Gamma_{t}^{r}} d \Gamma_{t}^{r} .
$$

The weak imposition of EBCs is expressed as the sum

$$
\begin{gathered}
a_{n i t}^{u}(\delta \boldsymbol{d}, \boldsymbol{d})-l_{n i t}^{u}(\delta \boldsymbol{d})=-\delta W_{n i t}^{u}, \\
a_{n i t}^{u}(\delta \boldsymbol{d}, \boldsymbol{d})=-\int_{\Gamma_{u}^{r}} \delta \boldsymbol{u} \cdot \boldsymbol{n}_{n}^{r} d \Gamma_{u}^{r}-\int_{\Gamma_{u}^{r}} \delta \boldsymbol{n}_{n}^{r} \cdot \boldsymbol{u} d \Gamma_{u}^{r}+\int_{\Gamma_{u}^{r}} \beta_{u}^{u} \delta \boldsymbol{u} \cdot \boldsymbol{u} d \Gamma_{u}^{r} \\
-\int_{\Gamma_{u}^{r}} \delta \boldsymbol{\theta} \cdot \boldsymbol{m}_{n}^{r} d \Gamma_{u}^{r}-\int_{\Gamma_{u}^{r}} \delta \boldsymbol{m}_{n}^{r} \cdot \boldsymbol{\theta} d \Gamma_{u}^{r}+\int_{\Gamma_{u}^{r}} \beta_{u}^{\theta} \delta \boldsymbol{\theta} \cdot \boldsymbol{\theta} d \Gamma_{u}^{r} \\
l_{n i t}^{u}(\delta \boldsymbol{d})=-\int_{\Gamma_{u}^{r}} \delta \boldsymbol{n} \cdot \overline{\boldsymbol{u}} d \Gamma_{u}^{r}+\int_{\Gamma_{u}^{r}} \beta_{u}^{u} \delta \boldsymbol{u} \cdot \overline{\boldsymbol{u}} d \Gamma_{u}^{r} \\
-\int_{\Gamma_{u}^{r}} \delta \boldsymbol{m} \cdot \overline{\boldsymbol{\theta}} d \Gamma_{u}^{r}+\int_{\Gamma_{u}^{r}} \beta_{u}^{\theta} \delta \boldsymbol{\theta} \cdot \overline{\boldsymbol{\theta}} d \Gamma_{u}^{r},
\end{gathered}
$$

and so are the interface terms

$$
\begin{gathered}
a_{n i t}^{i}(\delta \boldsymbol{d}, \boldsymbol{d})-l_{n i t}^{i}(\delta \boldsymbol{d})=-\delta W_{n i t}^{i}, \\
a_{n i t}^{i}(\delta \boldsymbol{d}, \boldsymbol{d})=-\int_{\Gamma_{i}^{r}}[\delta \boldsymbol{u}] \cdot\left\langle\boldsymbol{n}_{n}^{r}\right\rangle d \Gamma_{i}^{r}-\int_{\Gamma_{i}^{r}}\left\langle\delta \boldsymbol{n}_{n}^{r}\right\rangle \cdot[\boldsymbol{u}] d \Gamma_{i}^{r}+\int_{\Gamma_{i}^{r}} \beta_{i}^{u}[\delta \boldsymbol{u}] \cdot[\boldsymbol{u}] d \Gamma_{i}^{r} \\
-\int_{\Gamma_{i}^{r}}[\delta \boldsymbol{\theta}] \cdot\left\langle\boldsymbol{m}_{n}^{r}\right\rangle d \Gamma_{i}^{r}-\int_{\Gamma_{i}^{r}}\left\langle\boldsymbol{m}_{n}^{r}\right\rangle \cdot[\delta \boldsymbol{\theta}] d \Gamma_{i}^{r}+\int_{\Gamma_{i}^{r}} \beta_{i}^{\theta}[\delta \boldsymbol{\theta}] \cdot[\boldsymbol{\theta}] d \Gamma_{i}^{r} .
\end{gathered}
$$

Those components can be gathered

$$
\begin{aligned}
a_{n i t}(\delta \boldsymbol{d}, \boldsymbol{d}) & =a^{i n t}(\delta \boldsymbol{d}, \boldsymbol{d})+a_{n i t}^{u}(\delta \boldsymbol{d}, \boldsymbol{d})+a_{\text {nit }}^{i}(\delta \boldsymbol{d}, \boldsymbol{d}) \\
l_{n i t}(\delta \boldsymbol{d}) & =l^{e x t}(\delta \boldsymbol{d})+l_{n i t}^{u}(\delta \boldsymbol{d})+l_{n i t}^{i}(\delta \boldsymbol{d})
\end{aligned}
$$

and the equilibrium is expressed as

$$
a_{n i t}(\delta \boldsymbol{d}, \boldsymbol{d})=l_{n i t}(\delta \boldsymbol{d}) .
$$


We make use of the norms

$$
\begin{aligned}
\|\boldsymbol{a}\|_{\Omega^{r}}^{2} & =\int_{\Omega^{r}} \boldsymbol{a} \cdot \boldsymbol{a} d \Omega^{r} \text { in } \Omega^{r}, \\
\|\boldsymbol{a}\|_{\Gamma^{r}}^{2} & =\int_{\Gamma^{r}} \boldsymbol{a} \cdot \boldsymbol{a} d \Gamma^{r} \text { on } \Gamma^{r}
\end{aligned}
$$

and Young's inequality with $\epsilon$

$$
\boldsymbol{a} \cdot \boldsymbol{b} \leq \frac{\boldsymbol{a} \cdot \boldsymbol{a}}{2 \epsilon}+\frac{\epsilon \boldsymbol{b} \cdot \boldsymbol{b}}{2}
$$

so that

$$
\begin{aligned}
a_{n i t}^{u}(\delta \boldsymbol{d}, \delta \boldsymbol{d})= & -2 \int_{\Gamma_{u}^{r}} \delta \boldsymbol{u} \cdot \delta \boldsymbol{n}_{n}^{r} d \Gamma_{u}^{r}+\beta_{u}^{u} \int_{\Gamma_{u}^{r}} \delta \boldsymbol{u} \cdot \delta \boldsymbol{u} d \Gamma_{u}^{r} \\
& -2 \int_{\Gamma_{u}^{r}} \delta \boldsymbol{\theta} \cdot \delta \boldsymbol{m}_{n}^{r} d \Gamma_{u}^{r}+\beta_{u}^{\theta} \int_{\Gamma_{u}^{r}} \beta_{u}^{\theta} \delta \boldsymbol{\theta} \cdot \boldsymbol{\theta} d \Gamma_{u}^{r} \\
a_{n i t}^{u}(\delta \boldsymbol{d}, \delta \boldsymbol{d}) \geq & -\epsilon_{u}^{u}\|\delta \boldsymbol{u}\|_{\Gamma_{u}^{r}}^{2}-\frac{1}{\epsilon_{u}^{u}}\left\|\delta \boldsymbol{n}_{n}^{r}\right\|_{\Gamma_{u}^{r}}^{2}+\beta_{u}^{u}\|\delta \boldsymbol{u}\|_{\Gamma_{u}^{r}}^{2} \\
& -\epsilon_{\theta}^{u}\|\delta \boldsymbol{\theta}\|_{\Gamma_{u}^{r}}^{2}-\frac{1}{\epsilon_{\theta}^{u}}\left\|\delta \boldsymbol{m}_{n}^{r}\right\|_{\Gamma_{u}^{r}}^{2}+\beta_{u}^{\theta}\|\delta \boldsymbol{\theta}\|_{\Gamma_{u}^{r}}^{2} .
\end{aligned}
$$

for the essential boundary and

$$
\begin{aligned}
a_{n i t}^{i}(\delta \boldsymbol{d}, \delta \boldsymbol{d})= & -2 \int_{\Gamma_{i}^{r}}[\delta \boldsymbol{u}] \cdot\left\langle\delta \boldsymbol{n}_{n}^{r}\right\rangle d \Gamma_{i}^{r}+\beta_{i}^{u} \int_{\Gamma_{i}^{r}}[\delta \boldsymbol{u}] \cdot[\delta \boldsymbol{u}] d \Gamma_{i}^{r} \\
& -2 \int_{\Gamma_{i}^{r}}[\delta \boldsymbol{\theta}] \cdot\left\langle\delta \boldsymbol{m}_{n}^{r}\right\rangle d \Gamma_{i}^{r}+\beta_{i}^{\theta} \int_{\Gamma_{i}^{r}}[\delta \boldsymbol{\theta}] \cdot[\delta \boldsymbol{\theta}] d \Gamma_{i}^{r} \\
a_{n i t}^{i}(\delta \boldsymbol{d}, \delta \boldsymbol{d}) \geq & -\epsilon_{u}^{i}\|[\delta \boldsymbol{u}]\|_{\Gamma_{i}^{r}}^{2}-\frac{1}{\epsilon_{u}^{i}}\left\|\left\langle\delta \boldsymbol{n}_{n}^{r}\right\rangle\right\|_{\Gamma_{i}^{r}}^{2}+\beta_{i}^{u}\|[\delta \boldsymbol{u}]\|_{\Gamma_{i}^{r}}^{2} \\
& -\epsilon_{\theta}^{i}\|[\delta \boldsymbol{\theta}]\|_{\Gamma_{i}^{r}}^{2}-\frac{1}{\epsilon_{\theta}^{i}}\left\|\left\langle\delta \boldsymbol{m}_{n}^{r}\right\rangle\right\|_{\Gamma_{i}^{r}}^{2}+\beta_{i}^{\theta}\|[\delta \boldsymbol{\theta}]\|_{\Gamma_{i}^{r}}^{2} .
\end{aligned}
$$

We resort to distribution dependent relations (see [27] and [52]) between stains in the domain and reactions on the boundaries:

$$
\begin{array}{r}
\left\|\delta \boldsymbol{n}_{n}^{r}\right\|_{\Gamma_{u}^{r}}^{2} \leq C_{u}^{u} a^{i n t}(\delta \boldsymbol{d}, \delta \boldsymbol{d}) \\
\left\|\delta \boldsymbol{m}_{n}^{r}\right\|_{\Gamma_{u}^{r}}^{2} \leq C_{\theta}^{u} a^{i n t}(\delta \boldsymbol{d}, \delta \boldsymbol{d}) \\
\left\|\left\langle\delta \boldsymbol{n}_{n}^{r}\right\rangle\right\|_{\Gamma_{i}^{r}}^{2} \leq C_{u}^{i} a^{i n t}(\delta \boldsymbol{d}, \delta \boldsymbol{d}) \\
\left\|\left\langle\delta \boldsymbol{m}_{n}^{r}\right\rangle\right\|_{\Gamma_{i}^{r}}^{2} \leq C_{\theta}^{i} a^{i n t}(\delta \boldsymbol{d}, \delta \boldsymbol{d}),
\end{array}
$$

where the superscript $u$ indicate evaluation on the essential boundary, $i$ on an interface, for the subscripts, $u$ is related to displacements and $\theta$ to the rotations. Constants $C$ are positive and particular to each distribution. 
With these definitions,

$$
\begin{aligned}
a_{n i t}^{u}(\delta \boldsymbol{d}, \delta \boldsymbol{d}) \geq & -\frac{1}{\epsilon_{u}^{u}} C_{u}^{u} a^{i n t}(\delta \boldsymbol{d}, \delta \boldsymbol{d})+\left(\beta_{u}^{u}-\epsilon_{u}^{u}\right)\|\delta \boldsymbol{u}\|_{\Gamma_{u}^{r}}^{2} \\
& -\frac{1}{\epsilon_{\theta}^{u}} C_{\theta}^{u} a^{i n t}(\delta \boldsymbol{d}, \delta \boldsymbol{d})+\left(\beta_{u}^{\theta}-\epsilon_{\theta}^{u}\right)\|\delta \boldsymbol{\theta}\|_{\Gamma_{u}^{r}}^{2},
\end{aligned}
$$

and

$$
\begin{aligned}
a_{n i t}^{i}(\delta \boldsymbol{d}, \delta \boldsymbol{d}) \geq & -\frac{1}{\epsilon_{u}^{i}} C_{u}^{i} a^{i n t}(\delta \boldsymbol{d}, \delta \boldsymbol{d})+\left(\beta_{i}^{u}-\epsilon_{u}^{i}\right)\|\delta \boldsymbol{u}\|_{\Gamma_{i}^{r}}^{2} \\
& -\frac{1}{\epsilon_{\theta}^{i}} C_{\theta}^{u} a^{i n t}(\delta \boldsymbol{d}, \delta \boldsymbol{d})+\left(\beta_{i}^{\theta}-\epsilon_{\theta}^{i}\right)\|\delta \boldsymbol{\theta}\|_{\Gamma_{i}^{r}}^{2} .
\end{aligned}
$$

It can be said for the total bilinear form then

$$
\begin{aligned}
a_{n i t}(\delta \boldsymbol{d}, \delta \boldsymbol{d}) \geq & \left(1-\frac{C_{u}^{u}}{\epsilon_{u}^{u}}-\frac{C_{\theta}^{u}}{\epsilon_{\theta}^{u}}-\frac{C_{u}^{i}}{\epsilon_{u}^{i}}-\frac{C_{\theta}^{i}}{\epsilon_{\theta}^{i}}\right) a^{i n t}(\delta \boldsymbol{d}, \delta \boldsymbol{d}) \\
& +\left(\beta_{u}^{u}-\epsilon_{u}^{u}\right)\|\delta \boldsymbol{u}\|_{\Gamma_{u}^{r}}^{2}+\left(\beta_{u}^{\theta}-\epsilon_{\theta}^{u}\right)\|\delta \boldsymbol{\theta}\|_{\Gamma_{u}^{r}}^{2} \\
& +\left(\beta_{i}^{u}-\epsilon_{u}^{i}\right)\|[\delta \boldsymbol{u}]\|_{\Gamma_{i}^{r}}^{2}+\left(\beta_{i}^{\theta}-\epsilon_{\theta}^{i}\right)\|[\delta \boldsymbol{\theta}]\|_{\Gamma_{i}^{r}}^{2} .
\end{aligned}
$$

In order to Nitsche's bilinear form $a_{n i t}(\delta \boldsymbol{d}, \boldsymbol{d})$ to remain positive, each of the constants must obey relations in the form

$$
\beta \geq \varepsilon \geq 4 C
$$

We can take lower bounds for parameters $\beta$ from (4.76) as the distribution dependent inequalities $(4.72)$ provide a mean to estimate constants $C$. For each constant set, an eigenproblem of the form

$$
\mathbf{A} \boldsymbol{\delta}=\lambda \mathbf{K} \boldsymbol{\delta}
$$

is assembled. The largest eigenvalue is taken for $C$, and used for respective $\beta$ according to (4.76). Matrix $\mathbf{K}$ is the global stiffness matrix and a different matrix $\mathbf{A}$ must be assembled for the each eigenproblem. They are quite straightforward for EBCs,

$$
\begin{aligned}
& \mathbf{A}_{u}^{u}=\int_{\Gamma_{u}^{r}}\left(n_{\alpha} \mathbf{D}_{\alpha \beta}^{\eta} \mathbf{B}_{\beta}^{\eta}\right)^{T}\left(n_{\alpha} \mathbf{D}_{\alpha \beta}^{\eta} \mathbf{B}_{\beta}^{\eta}\right) d \Gamma_{u}^{r} \\
& \mathbf{A}_{\theta}^{u}=\int_{\Gamma_{u}^{r}}\left(n_{\alpha} \mathbf{D}_{\alpha \beta}^{\kappa} \mathbf{B}_{\beta}^{\kappa}\right)^{T}\left(n_{\alpha} \mathbf{D}_{\alpha \beta}^{\kappa} \mathbf{B}_{\beta}^{\kappa}\right) d \Gamma_{u}^{r}
\end{aligned}
$$


but require some assembling for the interfaces, as

$$
\begin{aligned}
\mathbf{A}^{i} & =\left[\begin{array}{cc}
\mathbf{A}_{11}^{i} & \mathbf{A}_{12}^{i} \\
\mathbf{A}_{21}^{i} & \mathbf{A}_{22}^{i}
\end{array}\right] \\
\mathbf{A}_{a b}^{i u} & =\int_{\Gamma_{i}^{r}}\left(n_{\alpha} \mathbf{D}_{\alpha \beta}^{\eta a} \mathbf{B}_{\beta}^{\eta a}\right)^{T}\left(n_{\alpha} \mathbf{D}_{\alpha \beta}^{\eta b} \mathbf{B}_{\beta}^{\eta b}\right) d \Gamma_{i}^{r} \\
\mathbf{A}_{a b}^{i \theta} & =\int_{\Gamma_{i}^{r}}\left(n_{\alpha} \mathbf{D}_{\alpha \beta}^{\kappa a} \mathbf{B}_{\beta}^{\kappa a}\right)^{T}\left(n_{\alpha} \mathbf{D}_{\alpha \beta}^{\kappa b} \mathbf{B}_{\beta}^{\kappa b}\right) d \Gamma_{i}^{r}, \\
& (a, b) \in[1,2], \text { with no sum over } a \text { or } b .
\end{aligned}
$$

In fact, matrices $\mathbf{A}^{i}$ can be expressed the same way as $\mathbf{A}^{u}$ if the global numbering for the degrees of freedom are taken into account in matrices $\mathbf{B}$. Since we use local numbering for the DOFs, we are compelled to separate into submatrices.

Although the eigenvalue problem is called global, not all degrees of freedom are involved in boundary approximation. Matrices $\mathbf{A}$ are only assembled for the DOFs related to particles whose support intersect the essential boundary. Only the subset of matrix $\mathbf{K}$ relating to these DOFs is used as the right-hand side of Eq. 4.77.

We propose the use of stabilization parameters in the form

$$
\beta=\alpha \max (\lambda)
$$

where $\max (\lambda)$ is the maximum eigenvalue of (4.77) and $\alpha \geq 4$ is a value in the same order of magnitude as 4 . 


\section{Chapter 5}

\section{Numerical Examples}

The numerical examples presented here serve to illustrate the applicability of the developed formulation for the analysis of shells of different thicknesses. First, it is shown that the eigenproblems (4.77) provide a good estimative for the stabilization parameters. Than, more complex geometries are analyzed.

\subsection{Stabilization for EBCs}

Two simple problems are used to demonstrate that our methodology provide good estimates for constants $\beta^{u}$.

\subsubsection{Displacement DOFs}

We start by analyzing the influence of the essential boundary stabilization constant for displacements $\beta_{u}^{u}$. The square $2 \times 2$ domain in Fig. 5.1 is discretized with $4 \times 4$ integration cells depicted as the black squares. Approximation nodes, blue circles, are positioned on the vertices of the integration cells. The plate is fixed on its left border and tractioned

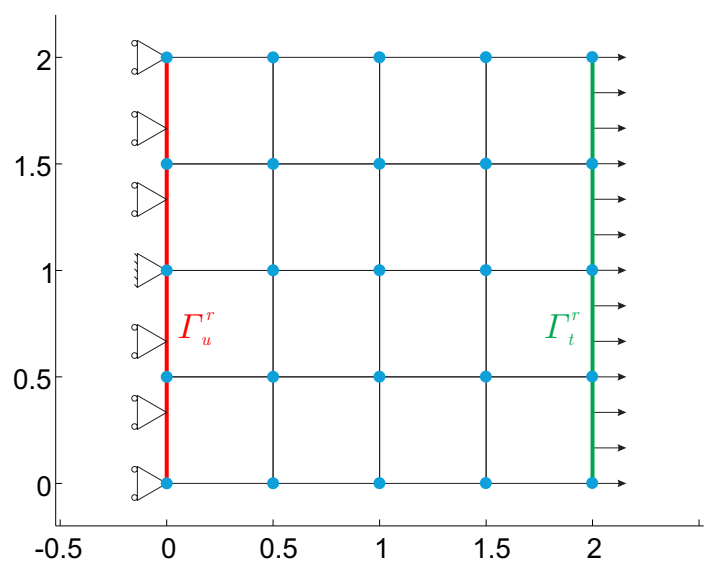

Figure 5.1: Plate under tension, single region. 


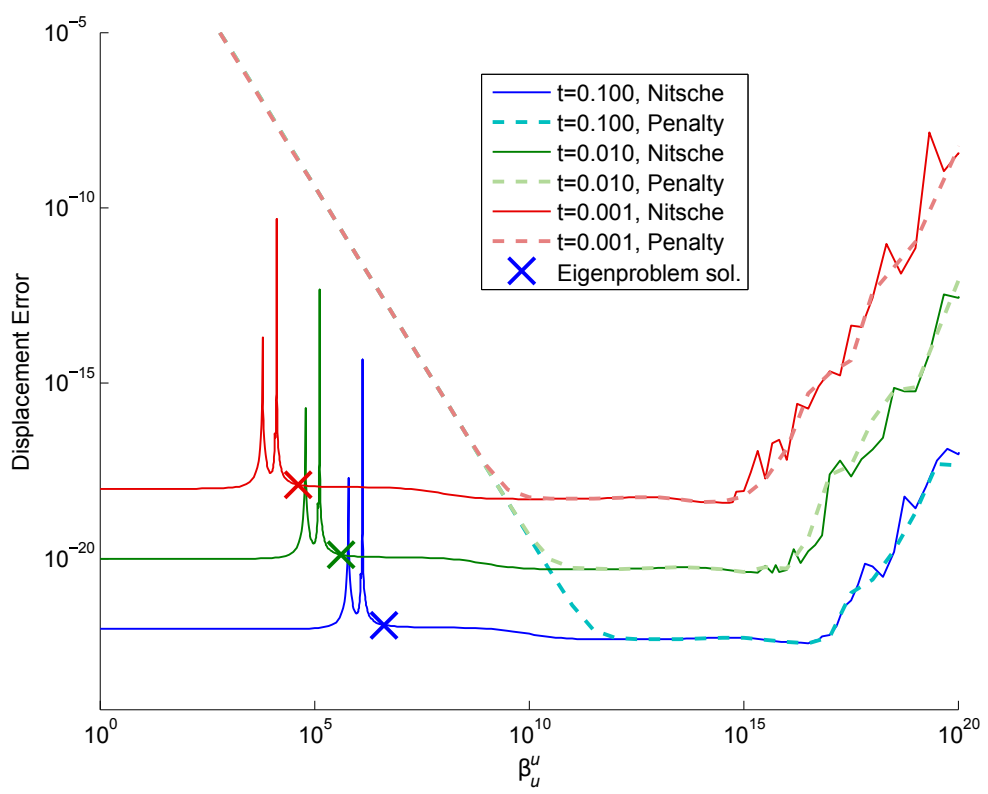

Figure 5.2: Plate under tension, single region. Displacement Error

by a unitary distributed load on the right boundary. Material parameters are $E=1 \times 10^{6}$ and $\nu=0.3$ and three thickness values $\left(2 \times 10^{-1}, 2 \times 10^{-2}\right.$ and $\left.2 \times 10^{-3}\right)$ are used

Different values of $\beta_{u}^{u}$ are employed for the Penalty and the Interior Penalty methods and results are compared in Fig. 5.2. The displacement error is computed across the domain as

$$
\epsilon_{d i s p}=\int_{\Omega^{r}}(\boldsymbol{d}-\overline{\boldsymbol{d}})^{2} d \Omega^{r}
$$

and $\overline{\boldsymbol{d}}$ is the analytical solution. Likewise, the boundary error is computed as

$$
\epsilon_{b o u n d}=\int_{\Gamma_{u}^{r}}(\boldsymbol{d}-\overline{\boldsymbol{d}})^{2} d \Gamma_{u}^{r}
$$

and shown in Fig. 5.3. The crossed marks in both figures represent the eigenvalue obtained from Eqs. 4.77 and 4.78a for each shell thickness and the respective error when using this values as $\beta_{u}^{u}(\alpha=1)$.

It is important to notice that the area of suitable constants for Nitsche's method, where the boundary error is equivalent to the approximation error, is much broader than that for the penalty method, as it is merely a stabilization parameter. For the penalty method, the boundary error in Fig. 5.3 is inversely proportional to the parameter up to values that render the system as ill-conditioned.

Also, the eigenvalue obtained is a good approximation for the frontier between the stable and unstable responses. For this very simple example, any $1 \leq \alpha \leq 1 \times 10^{15}$ should render good results. 


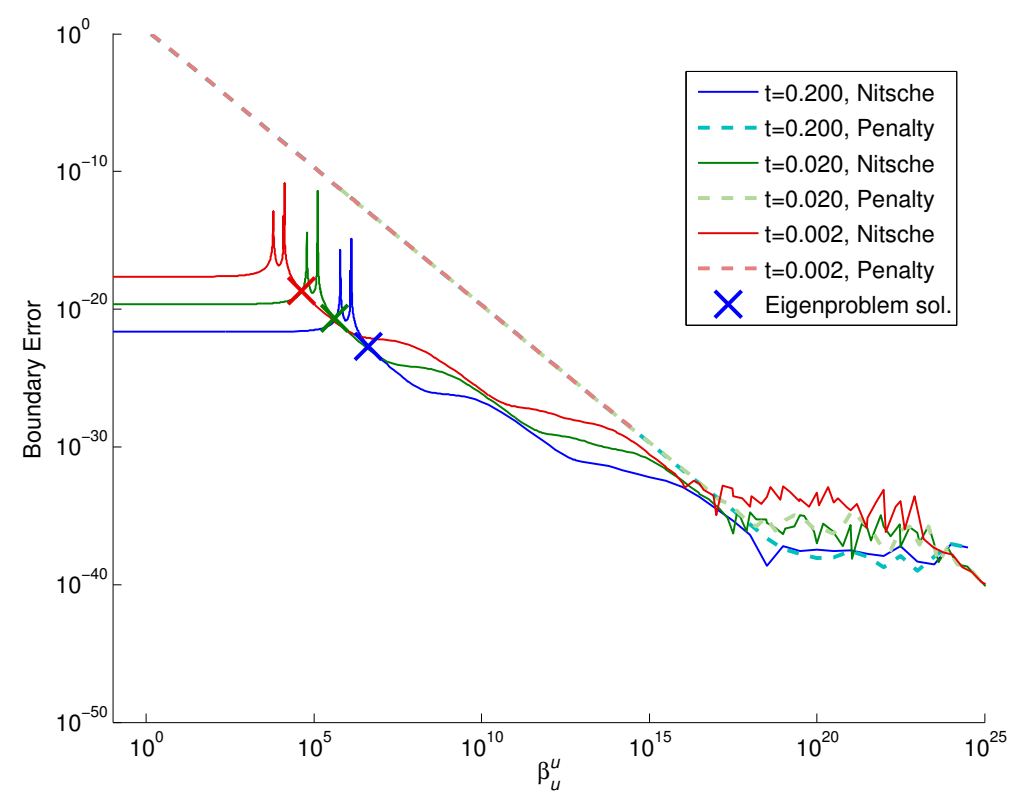

Figure 5.3: Plate under tension, single region. Essential Boundary Error

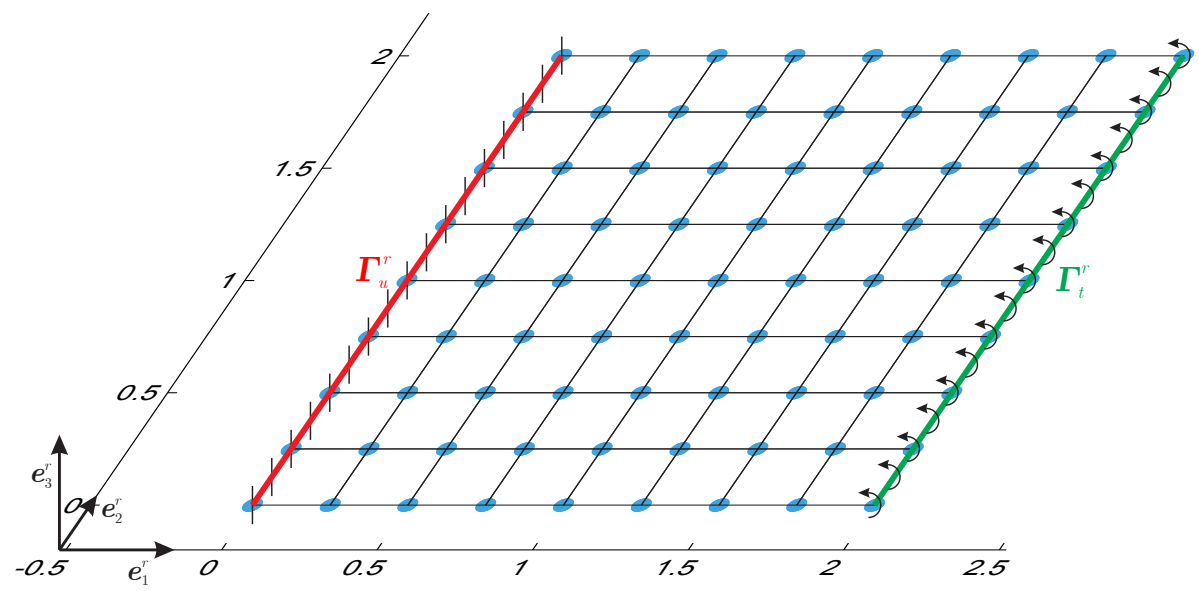

Figure 5.4: Plate under pure bending, single region.

\subsubsection{Rotation DOFs}

A similar model is used for assessing the constant for rotational degrees of freedom. A denser particle distribution is depicted in Fig. 5.4 so the domain approximation error doesn't play a significant role in the analysis.

The material parameters are $E=1 \times 10^{6}$ and $\nu=0.0$ and the same three thicknesses as in the first example are used. We keep the boundary stabilization parameter for displacements fixed as eigenvalue obtained from (4.78a) with an amplification value of $\alpha_{u}^{u}=10$.

Domain displacement results for the bending parameter are shown in Fig. 5.5 and boundary error in Fig. 5.6. The same observations made for the previous example can be made for the rotational DOFs, but for a slightly narrower suitable range for stabilization parameter. Nevertheless, the eigenvalue is still a good approximation. 


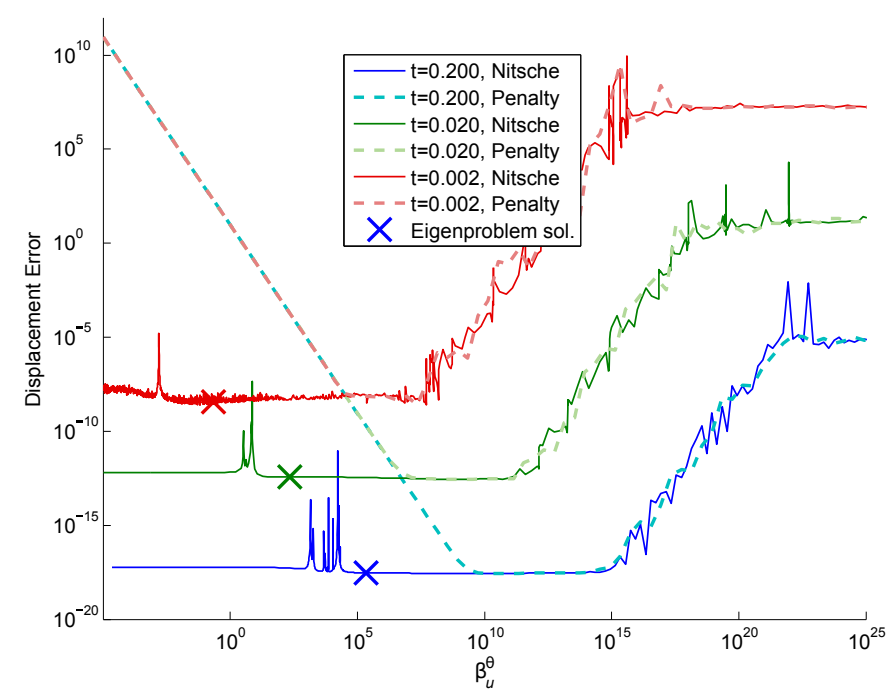

Figure 5.5: Plate under pure bending, single region. Displacement Error

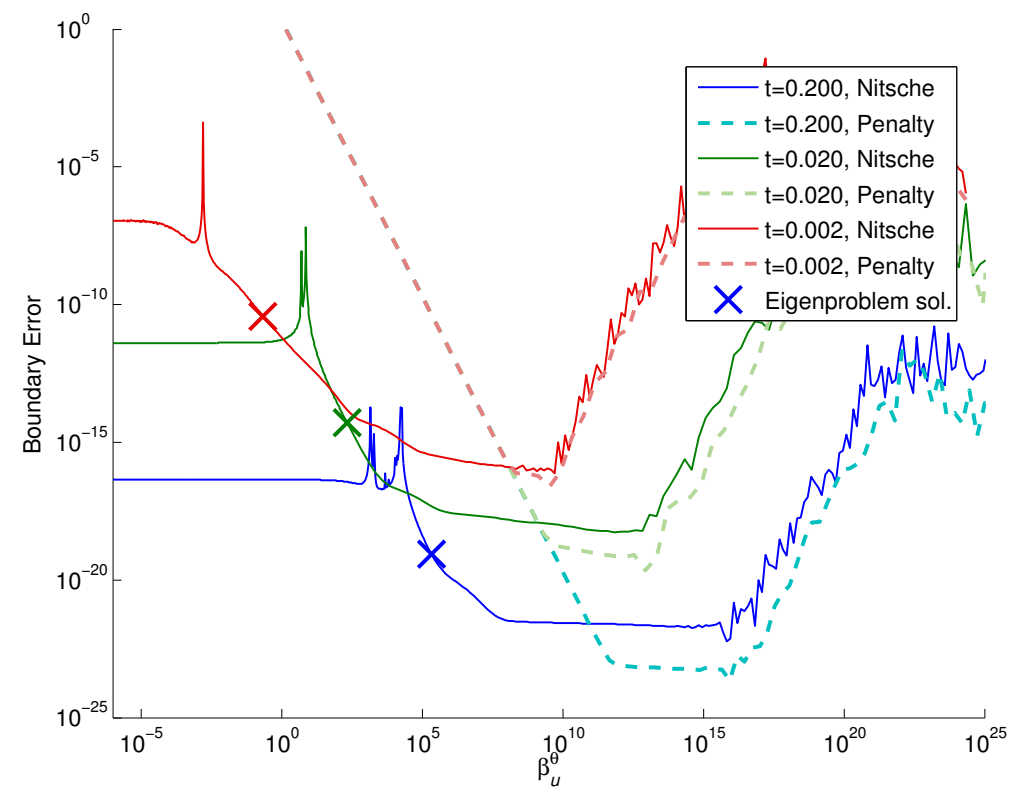

Figure 5.6: Plate under pure bending, single region. Essential Boundary Error 


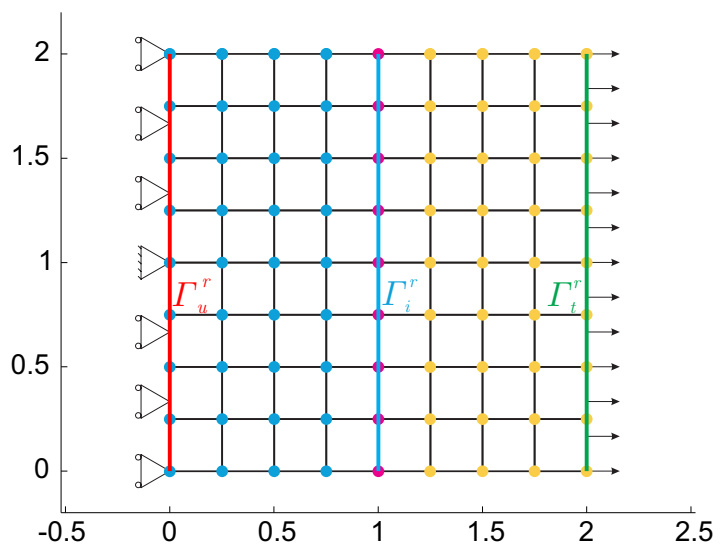

Figure 5.7: Plate under tension, two regions.

\subsection{Stabilization for Interfaces}

Similar problems are used to analyze the imposition of continuity and equilibrium across the interfaces. We start with simple models, analogous to the previous, whose approximation error we compare for different stabilization / penalty parameters. The previous domain is divided in two contiguous regions and both penalty and Nitsche's methods are employed on the interface.

The error on the interface is defined as

$$
\epsilon_{\text {intf }}=\int_{\Gamma_{i}^{r}}\left(\boldsymbol{d}_{1}-\boldsymbol{d}_{2}\right)^{2} d \Gamma_{i}^{r}
$$

where $\boldsymbol{d}_{n}$ is the approximation of the generalized displacements using the approximation of region $n$.

\subsubsection{Displacement DOFs}

For the analysis of the displacement DOFs, once again a pure tension plate is analyzed. The particle distribution is shown in Fig. 5.7, where the blue dots are the particles used to approximate the DOFs of region 1, the yellow dots, for region 2 and the red dots are actually the superposition of particles of regions 1 and 2, whose DOFs are independent. The blue line is the interface. Material parameters are kept and shells of three different thicknesses are analyzed.

The objective is to analyze the interface constraints, so EBCs kept constant, imposed with Nitsche's method, with stabilization parameters $\beta_{u}^{u}$ and $\beta_{u}^{\theta}$ as the maximum eigenvalue of $(4.78 \mathrm{a}, 4.78 \mathrm{~b})$ amplified by $\alpha^{u}=10$ and constant for each thickness.

$\beta_{i}^{\theta}$ is also kept constant at the maximum eigenvalue of $(4.79 \mathrm{c})$, so that only $\beta_{i}^{u}$, the stabilization parameter for the displacement DOFs at the interface, influences the results.

The results are shown in Fig. 5.8 for the domain error and Fig. 5.9 for the error 


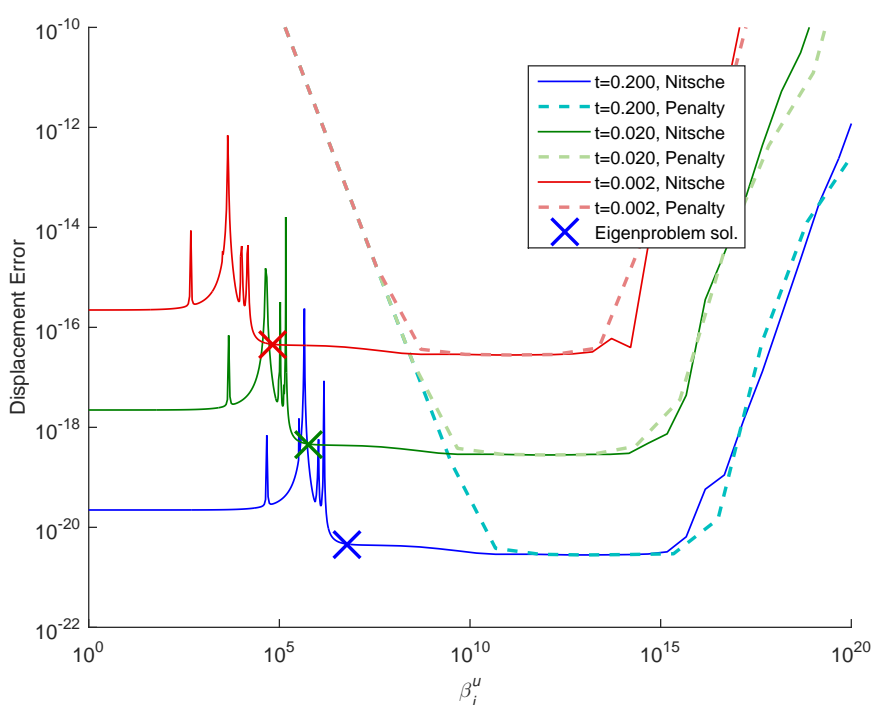

Figure 5.8: Plate under tension, two regions. Displacement Error

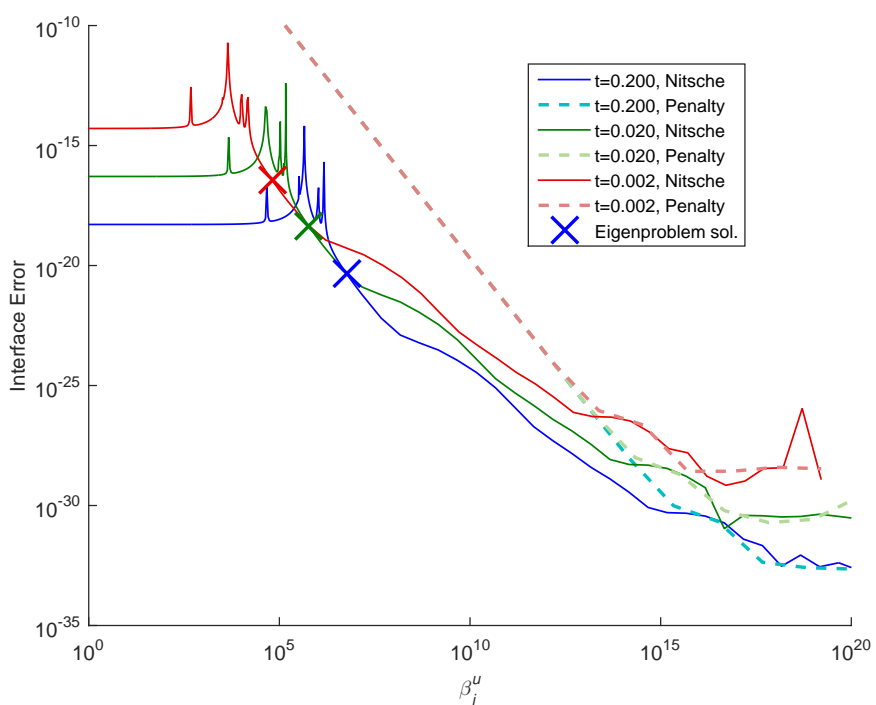

Figure 5.9: Plate under tension, two regions. Interface Error 


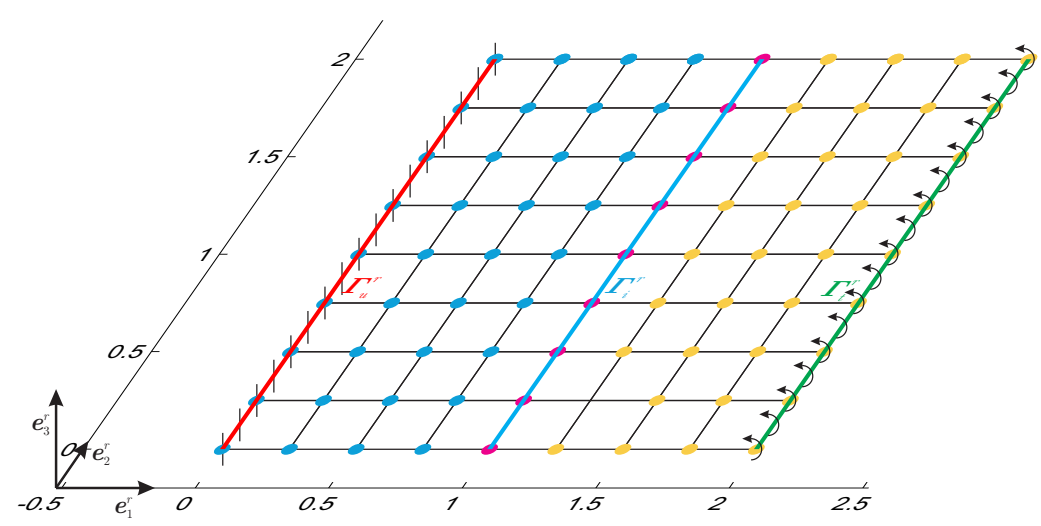

Figure 5.10: Plate under pure bending, two regions.

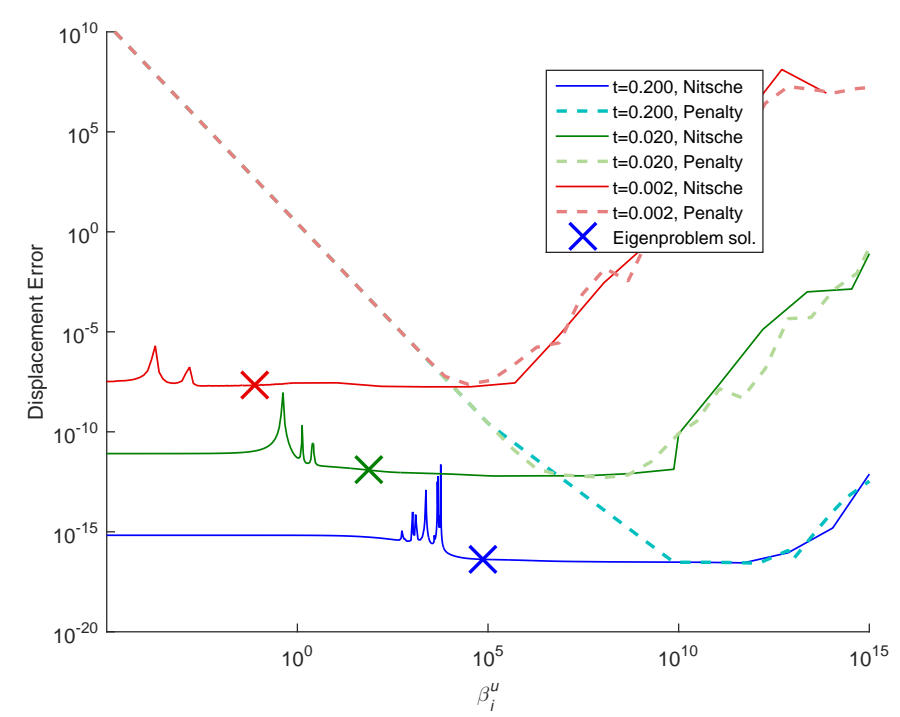

Figure 5.11: Plate under pure bending, two regions. Displacement Error

on the interface. Once again, the maximum eigenvalue of the proposed system correctly approximates the end of the unstable zone and the baselines of the error curves are wide enough to accommodate values of $1 \leq \alpha \leq 1 \times 10^{7}$ approximately.

\subsubsection{Rotation DOFs}

For the particle distribution in Fig. 5.10, several values of $\beta_{i}^{\theta}$ where used as to assess the error in the interface continuity. The values of $\beta_{u}^{u}$ and $\beta_{u}^{\theta}$ were taken according to (4.80) with $\alpha=10$ and for $\beta_{i}^{u}, \alpha=1$.

The results are presented in Figs. 5.11, 5.12 and similar conclusions can be drawn. The solution of the proposed eigenproblem is clear of the perturbed region by a considerable margin, still away of the numerical instabilities of a pure penalty approach. 


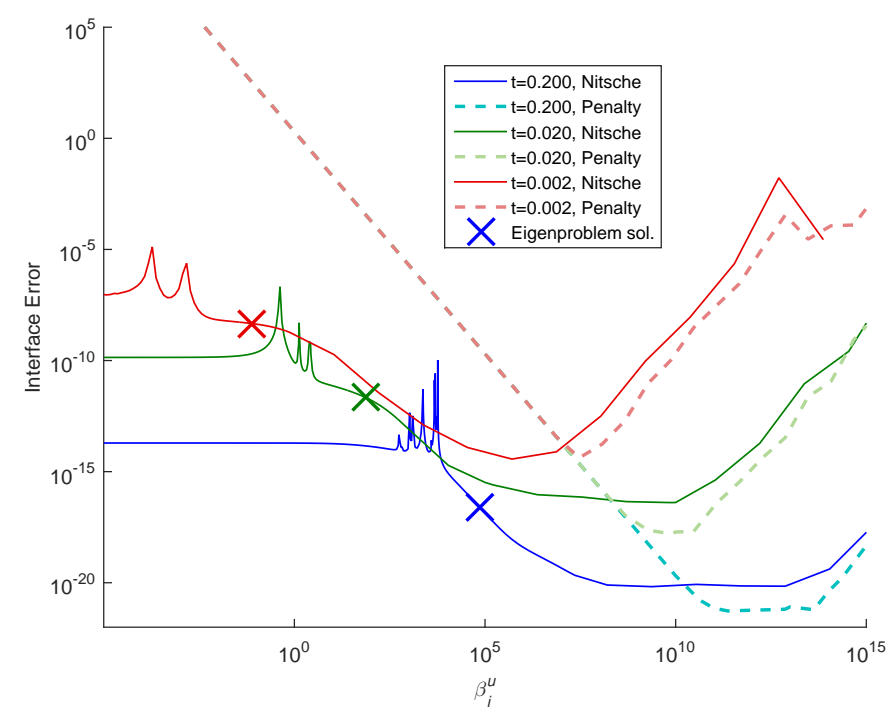

Figure 5.12: Plate under pure bending, single region. Essential Boundary Error

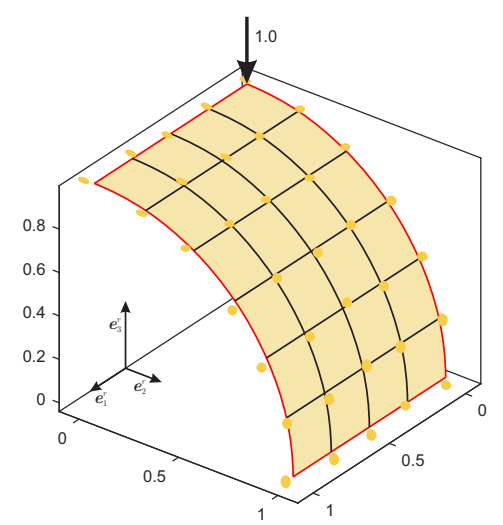

(a)

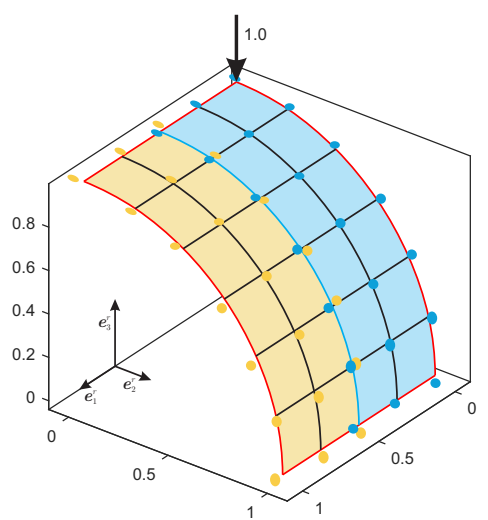

(b)

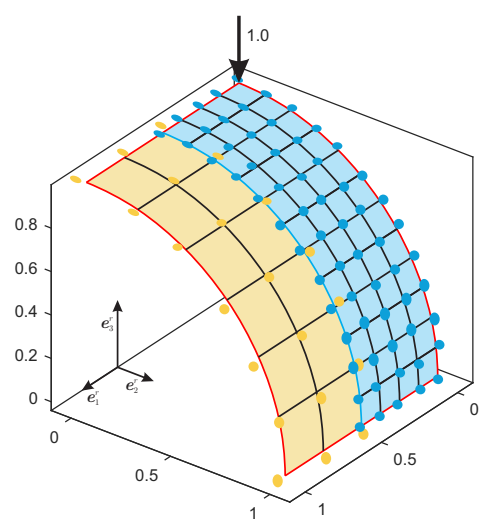

(c)

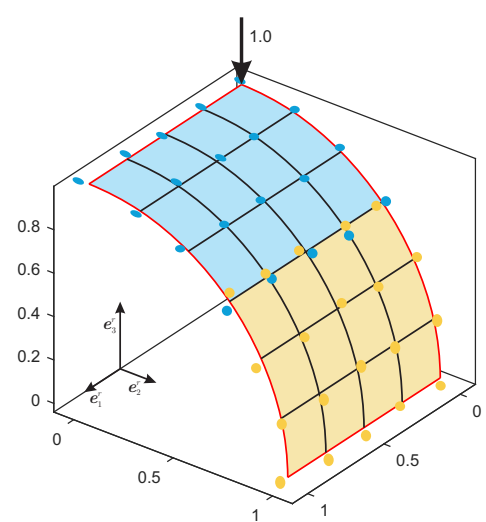

(d)

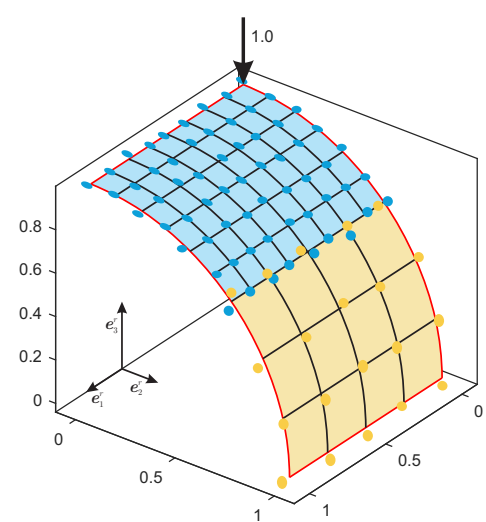

(e)

Figure 5.13: Pinched Cylinder. Discretization models $A, B$ and $C$ respectively 


\subsection{Pinched Cylinder}

The classical Pinched Cylinder [56] example is presented next. It consists of a cylindrical shell with rigid diaphragms on each end subject to two opposing concentrated loads pinching its center point inwards. For simplicity, we model only one eighth of the cylinder, depicted in Fig. 5.13. The shell has a thickness of 0.01, Young modulus of $9^{8}$ and Poisson ratio of 0.3 . The length of the full cylinder is 2.0 and its radius 1.0. The applied load is $1 / 1200$ downwards.

The MLS shape functions were constructed using a fifth order polynomial base and a support radius amplified by 3 . The use of a high-order base is demanding on the particle distribution concerning linear dependencies. The particles are then moved from a straight structured distribution by a small random perturbation. Particles may lay outside the approximation domain, as long as their support covers at least part of it. In this case, the particles don't coincide with the nodes of the integration cell discretization depicted in Fig. 5.13.

Five different models were developed and compared. Model A uses a single region with almost uniform particle distribution and no interface. Model B divides the domain into two regions through a interface at $\xi_{1}=0.5$ with both regions approximated by similar particle distributions. It serves to demonstrate that the present framework can reproduce curved interfaces. Model C, also uses two discretization regions, but with the blue region more refined than the yellow region. This illustrates the possibility of local refinement, equivalent to non conforming FE meshes. Models D and E have similar definitions as B and $\mathrm{C}$, but with a longitudinal interface. Particle distributions for models A, B, C, D and E are all depicted in Fig. 5.13.

Kinematic constraints are enforced either with Lagrange Multipliers or with Nitsche's Method using the eigenvalue problem solution and all $\alpha=10$. For the Lagrange Multipliers, the linear solver warned for badly scaled system matrix if the trivial choice of discretization was chosen for the interface and the essential boundary. As results were similar to the ones obtained with Nitsche's method for similar particle distributions, we trust that the MATLAB solver could handle the badly scaled system.

Fig. 5.14 shows the vertical displacement of the loaded point. Results converge to the reference solution $-6.0829 \times 10^{-8}$, with the same ratio for Nitsche's method as for Lagrange multipliers.

It is noticeable that Model B converges with similar ratio as Model A, but requires more DOFs to achieve the same error. This is expected as the use of two regions with same approximation properties brings no significant advantage in this case. The approximation space is not enriched in this area.

As for Model C, convergence is kept at a similar ratio, but again we need too many DOFs to achieve the same level of error. Even thought Nitsche's Method precludes the 


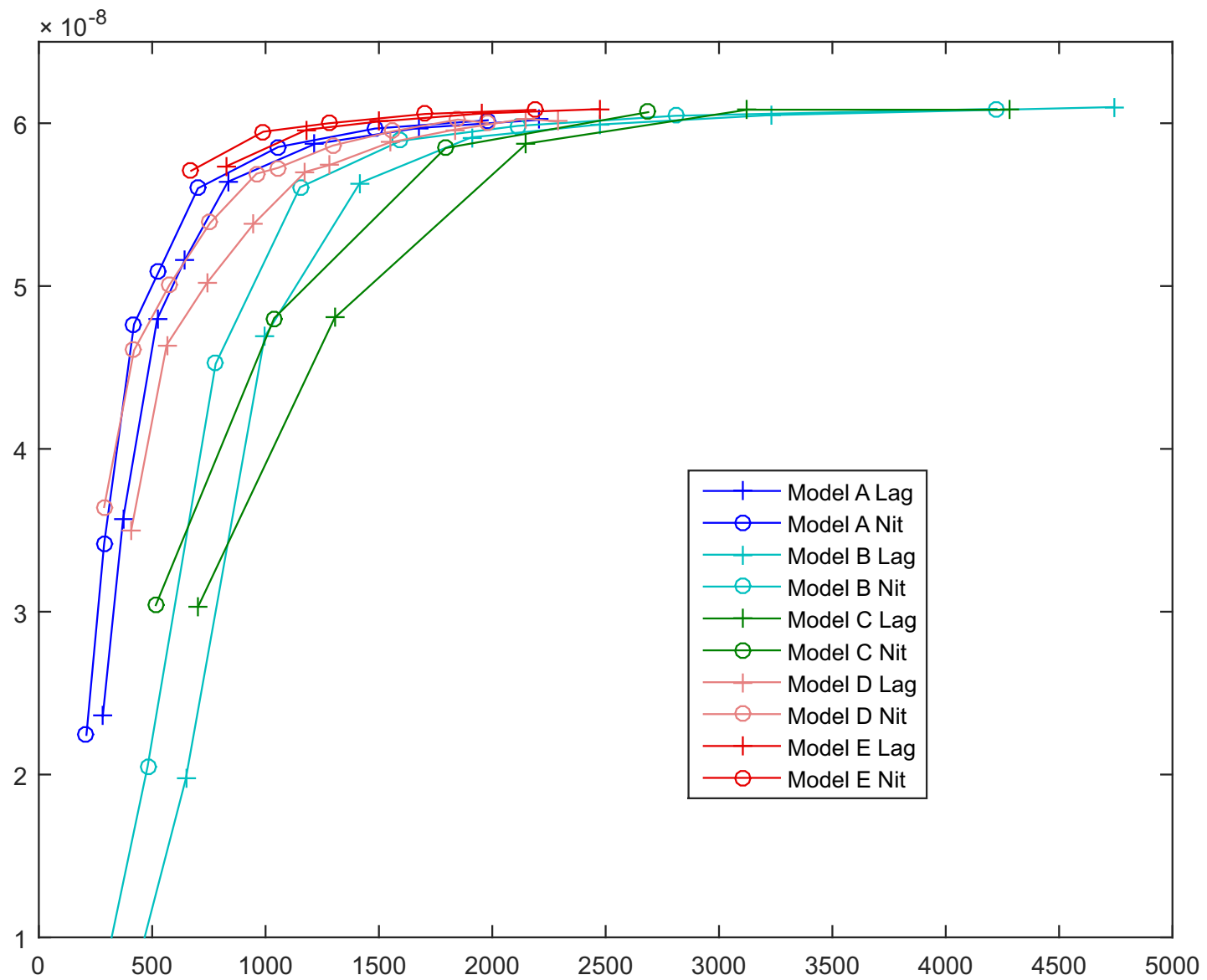

Figure 5.14: Pinched Cylinder. Loaded point displacements 


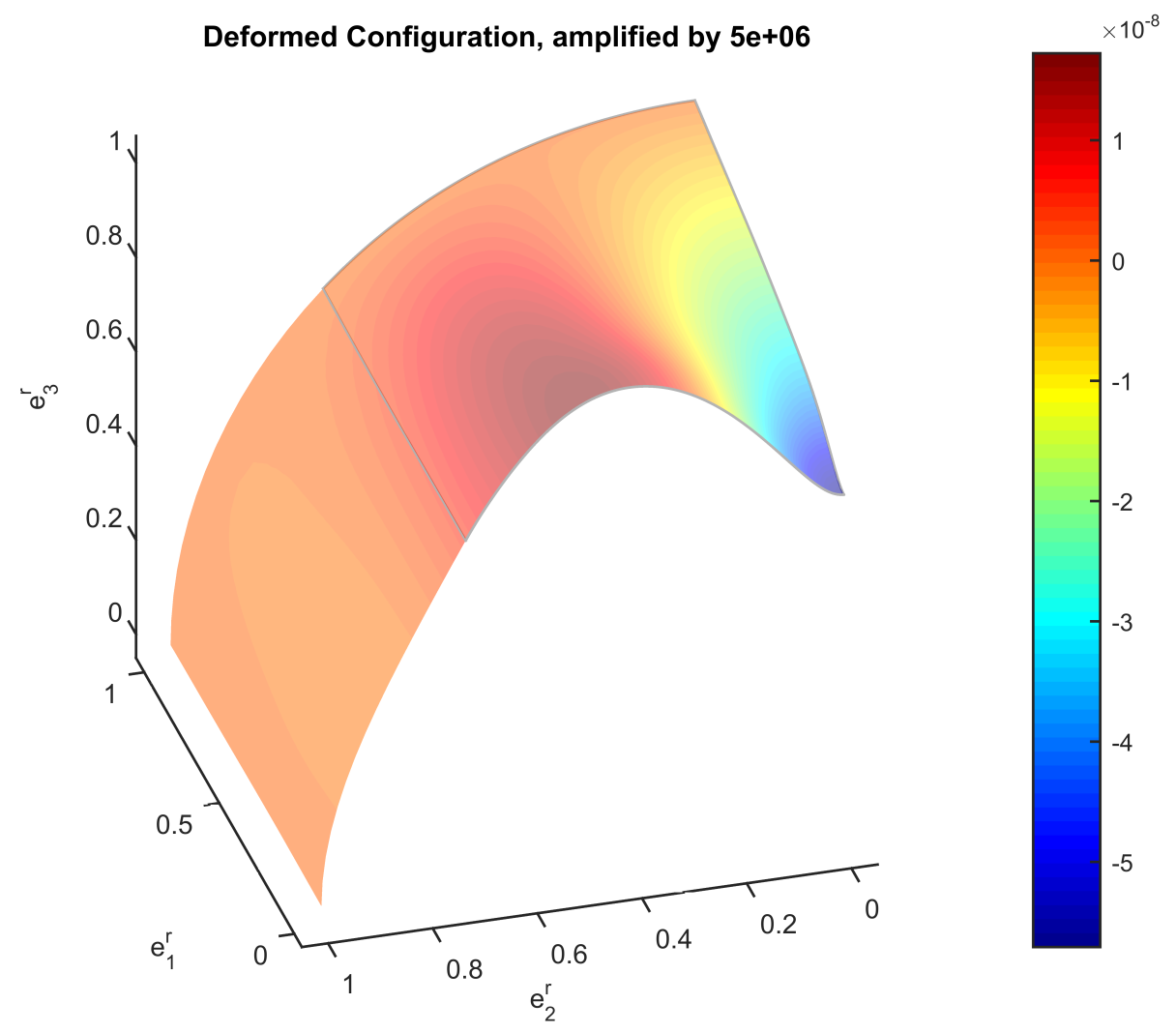

Figure 5.15: Pinched Cylinder. Deformed shape

introduction of interface DOFs, the separate approximations requires the nodes on the interface to be doubled. These extra DOFs are also present in the Lagrange Multiplier framework as it relates to the discretization rather then to the kinematic constraint.

Models D and E, with a shorter interface, don't need so many DOFs introduced. Model E presents the best results for a same number of DOFs as refinement is carried out in the area with higher gradients. A representation of the displacements on the converged solution is depicted in Fig. 5.15

\subsection{T-beam}

A balance T-beam under concentrated load is analyzed in [22] and reproduced in Fig. 5.16. Young modulus was taken as $3 \times 10^{6}$ and Poisson's ratio 0.3. This example presents a possibility to analyze a structure assembled from shells in different planes. The interface is the line on the intersection of the shells' midsurfaces.

The solution present in [22] and depicted in Fig. 5.16(a) includes a interface inside the domain. Despite departing from the described framework, it presents no difference in implementation and forms our 2-region model. Nevertheless, for the balance of moments on the intersection between the flange and the web to be kept, the moment acting on the 

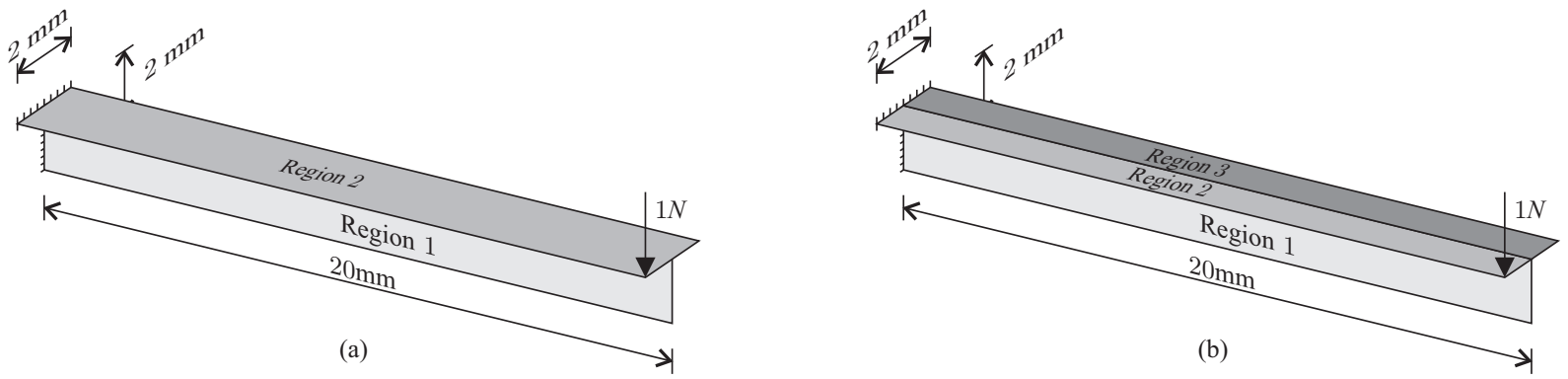

Figure 5.16: T-beam. (a) discretized by 2 regions. (b) discretized by 3 regions

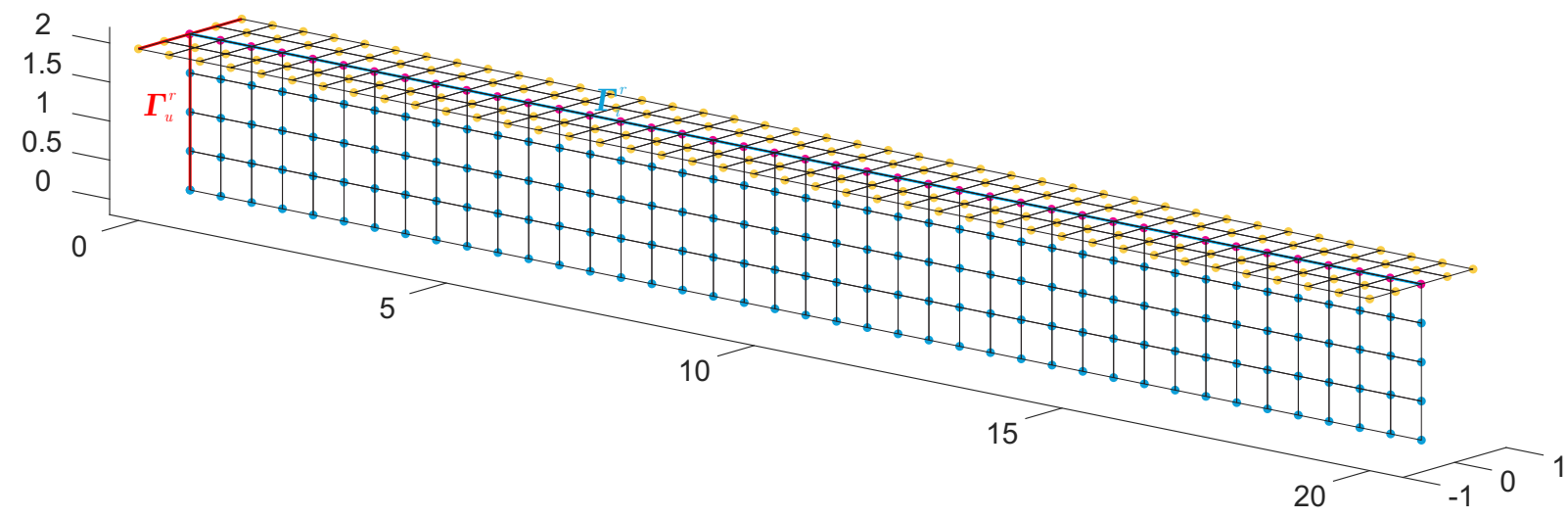

Figure 5.17: T-beam, two regions

flange should be discontinuous on the junction, what cannot be represented by a single region in the flange. For that, a 3-region model is presented in Fig. 5.16(b), where region 1 is the web and each of regions 2 and 3 represent one side of the flange.

A illustrative particle distribution is presented in 5.17 for the 2-region model, as the 3-region equivalent can be obtained by doubling the particles on the interface and dividing the set of particles on the flange in two. A quadratic base was used for the MLS approximant, with dilatation parameter 1.75 .

We compare the displacements on the loaded point for different particle distribution similar to Fig. 5.17. Either Lagrange Multipliers or Nitsche's Method are used simultaneously on the essential boundary and the interface. For the latter, the stabilization parameters are obtained from the global eigenvalue problem and magnified by $\alpha=10$. Both models converge similarly and independent from the method of imposing kinematic constraints.

An important objective for this model is to keep the right angle between the flange and the web. Normalized results are shown in Fig. 5.19 for Lagrange Multipliers and Nitsche's Method using the particle distribution depicted in Fig. 5.17. The graph on top presents the results for the 2-region model, the one below shows the angles between the pairs of region in the 3 -region model.

The angle is given by the different values of $\theta_{1}$ obtained in each region. Despite a significant difference between models and solution strategy, the error is kept below $1 \times 10^{-6}$ 


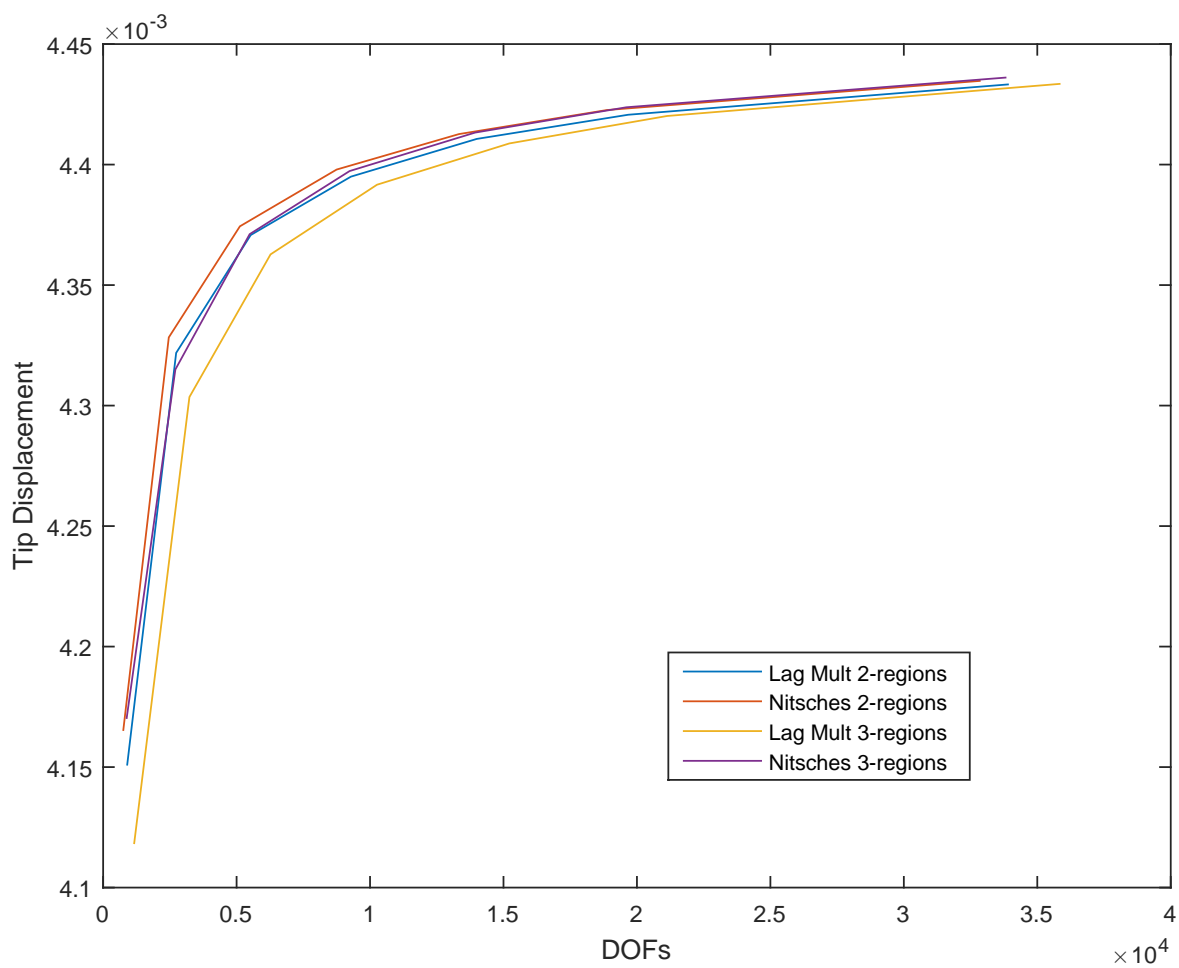

Figure 5.18: T-beam, tip displacement

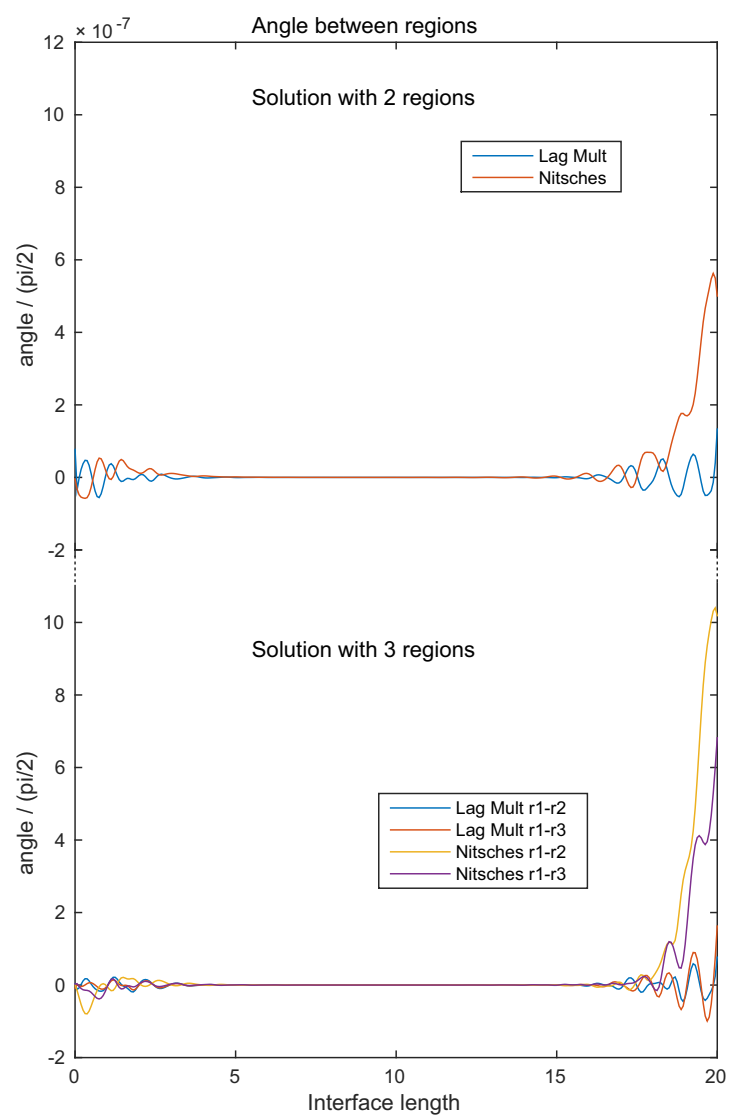

Figure 5.19: T-beam, angle between regions 


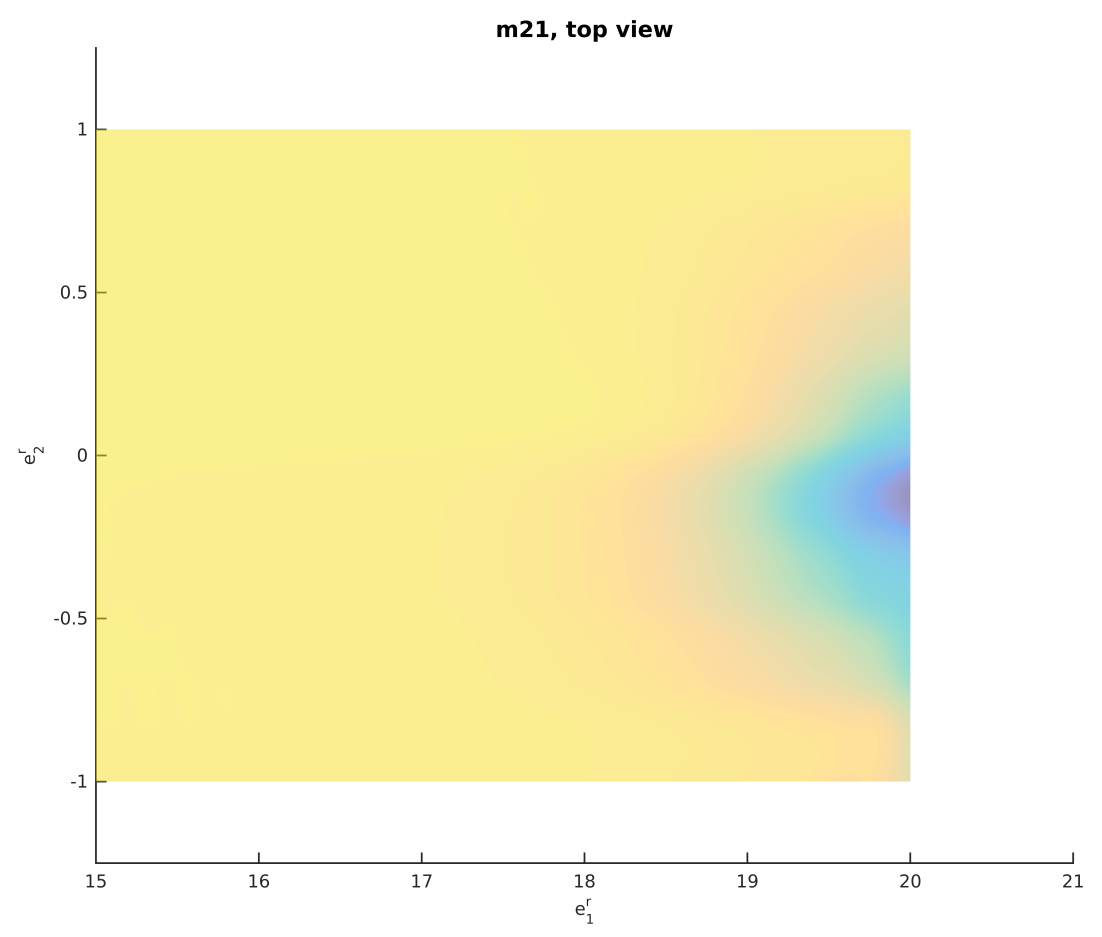

Figure 5.20: T-beam, moments for 2-region model

for the worst situation.

The 2-region model emulates the overall behavior really well, with compatible results for tip displacement, however cannot represent a sharp stress jump. Figs. 5.20-5.21 show a top view of the twisting moment $m_{21}$ for each approach and exemplifies how a 3-region model can be useful. Fig. 5.22 shows the deformed model with amplified displacements 


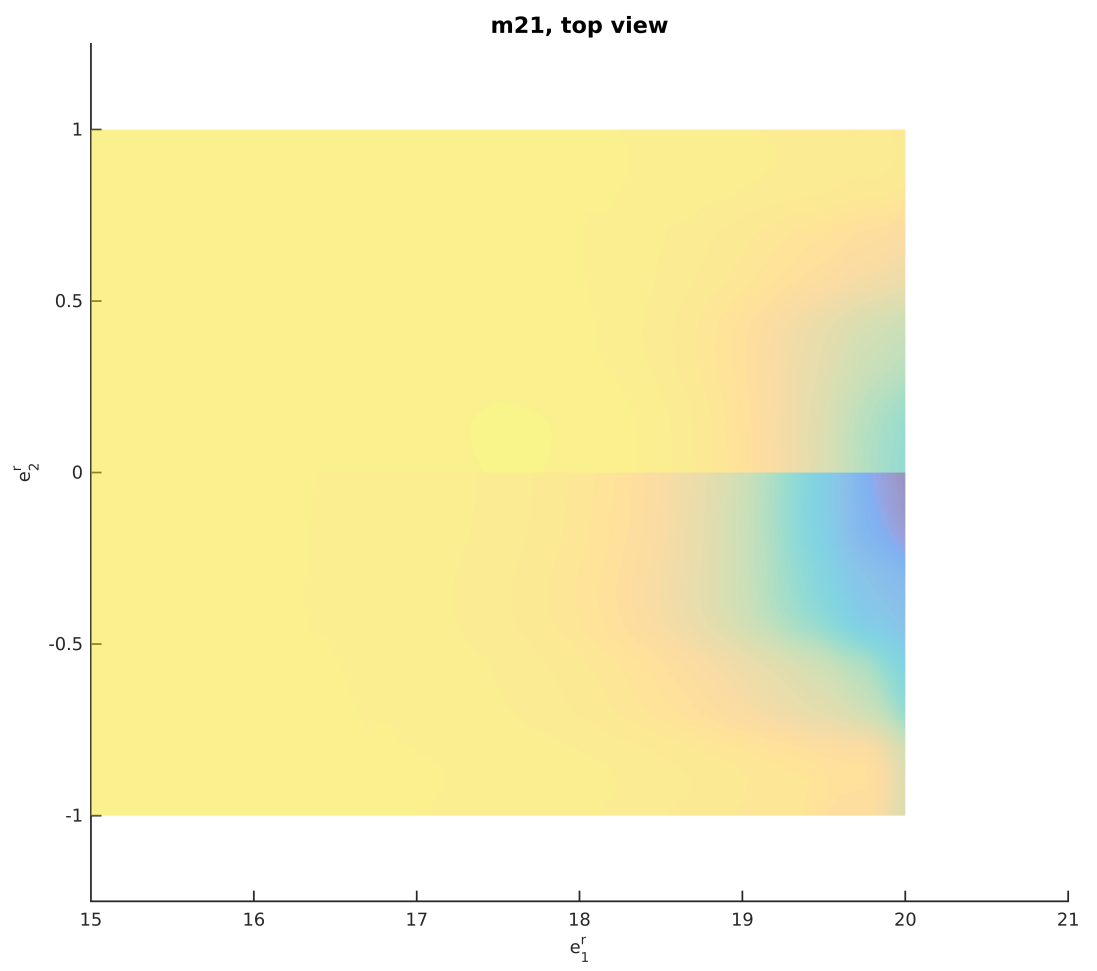

Figure 5.21: T-beam, moments for 3-region model

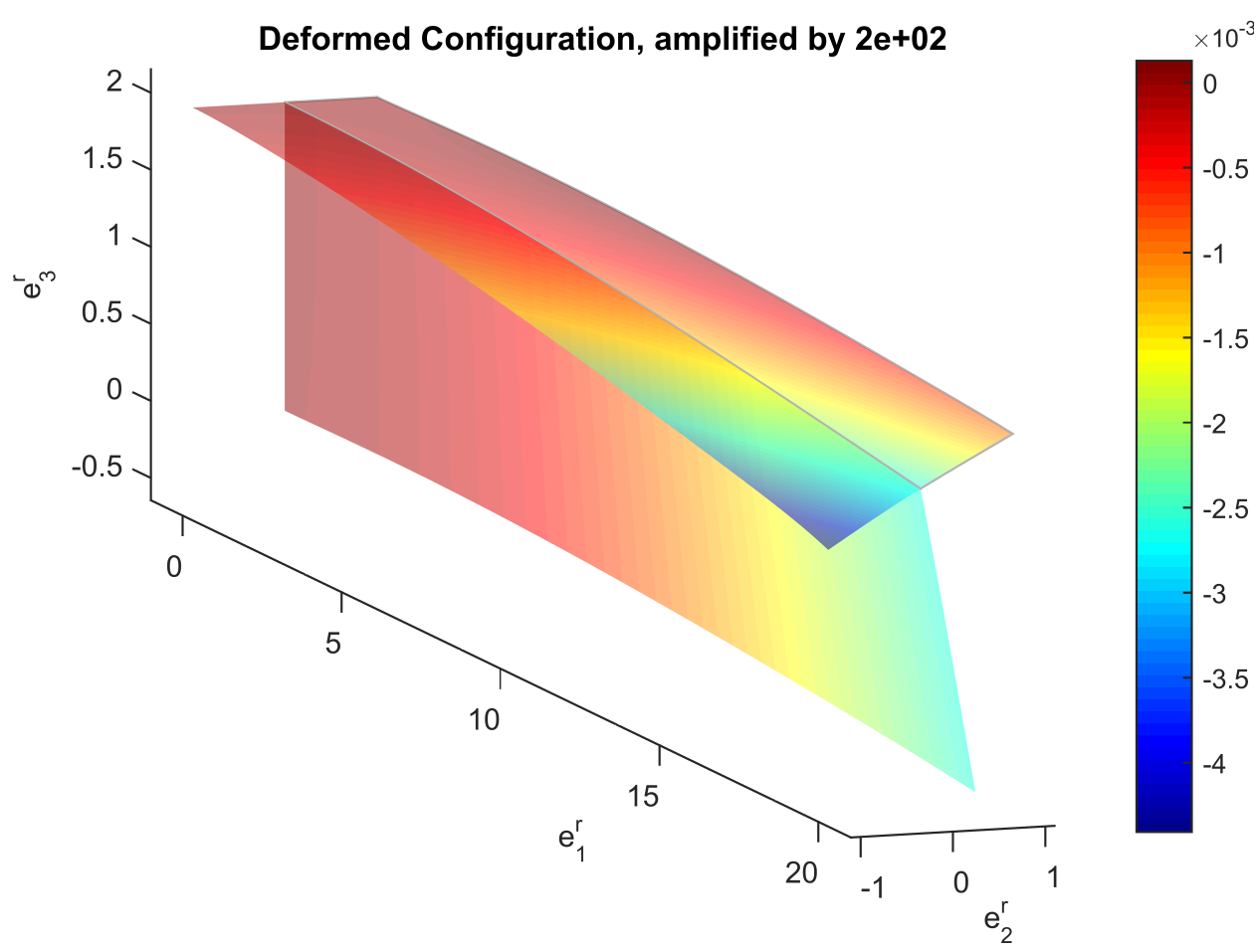

Figure 5.22: T-beam, displacements for 3-region model 


\section{Chapter 6}

\section{Conclusions}

This work has presented an alternative for imposing kinematic conditions in the linear analysis of shear deformable shells using the Element-Free Galerkin method: Nitsche's method. The approach is adapted to the shear deformable shell model and suitable for Element-free Galerkin analysis, accounting for rotation degrees of freedom and bending tractions. It is compared to more usual Penalty and Lagrange Multiplier methods, and consistency between weak and strong formulation is demonstrated. Numerical simulations present similar rates of convergence for the three methods, with no addition of degrees of freedom or loss of consistency for a wider range of stabilization parameters for Nitsche's approach.

One of the setbacks of Nitsche's Method is the presence of a user defined stabilization penalty-like parameter. The choice is not as fundamental as in a pure penalty parameter as the range of suitable values is wider. Nevertheless, the use of rotational degrees of freedom and bending stiffness, which assume different order of magnitude than the displacement DOFs and different dependency on the shell thickness, narrows the decision space if the parameter were to be taken as the same for all fields. We overcome this barrier proposing different parameters $\beta$ for each set of DOFs and proposing eigenvalue problems to estimate a lower bound. The matrices used for the eigenvalue problem are either already computed during domain assembly or have components already calculated for boundary imposition. Even more, only the higher eigenvalue is needed, so the assembly and solution of the eigenvalue problem is not so demanding during computation.

Even so, some measures could be implemented as to speed the process. If the approximations are similar (same material properties and similar particle spacing), the result obtained for one boundary can be used on the others and the interfaces. A local approach for the eigenvalue problem is available for Finite Elements [15], where the mesh dependent inequalities are expressed in a single element's domain thus assembling and solving the eigenproblem with only one element's degrees of freedom. The stabilization parameter is then taken as the largest of the element-wise estimations or as variable on the interface. The lack of elements makes the extension to meshless methods not straightforward, 
but possible via boundary integration cells. These alternatives are left as possibilities for future research. Extension to nonlinear problems is another desired feature, as it would emphasize the advantage of a smaller system of equations.

Numerical results show that the method is efficient, with similar error and convergence properties as Lagrange Multipliers and more stable than the Penalty method. Flexibility was demonstrated as curved interfaces, folded shells and discretization with different particle density were accurately represented.

The developed model proves suitable for shell analysis under small displacement and rotations, keeping a neat formulation whose weak form correspond to the differential statements with no need for a secondary approximation field or a hybrid formulation. The extension of Nitsche's weak for to encompass rotational DOFs was successful and the estimation procedure for stabilization parameters renders a proper lower bound and thus a stable formulation.

These contributions were summarized and submitted for publication by Costa, Pimenta and Wriggers [11]. 


\section{Bibliography}

[1] C. Annavarapu, M. Hautefeuille, and J. E. Dolbow. A robust Nitsche's formulation for interface problems. Computer Methods in Applied Mechanics and Engineering, 225-228(0):44 - 54, 2012. 2, 32, 36

[2] C. Annavarapu, M. Hautefeuille, and J. E. Dolbow. Stable imposition of stiff constraints in explicit dynamics for embedded finite element methods. International Journal for Numerical Methods in Engineering, 92(2):206 - 228, 2012. 2

[3] C. Annavarapu, M. Hautefeuille, and J. E. Dolbow. A nitsche stabilized finite element method for frictional sliding on embedded interfaces. part ii: Intersecting interfaces. Computer Methods in Applied Mechanics and Engineering, 267:318 - 341, 2013. 2

[4] C. Annavarapu, M. Hautefeuille, and J. E. Dolbow. A Nitsche stabilized finite element method for frictional sliding on embedded interfaces. Part I: Single interface. Computer Methods in Applied Mechanics and Engineering, 268:417 - 436, 2014. 2

[5] T. Belytschko, Y. Krongauz, D. Organ, M. Fleming, and P. Krysl. Meshless methods: An overview and recent developments. Computer Methods in Applied Mechanics and Engineering, 139(1-4):3 - 47, 1996. 21

[6] T. Belytschko, Y. Y. Lu, and L. Gu. Element-free galerkin methods. International Journal for Numerical Methods in Engineering, 37(2):229-256, 1994. 1, 16, 20, 22

[7] T. Belytschko, D. Organ, and Y. Krongauz. A coupled finite element-element-free galerkin method. Computational Mechanics, 17(3):186-195, 1995. 2, 20

[8] F. Cirak, M. Ortiz, and P. Schröder. Subdivision surfaces: a new paradigm for thin-shell finite-element analysis. International Journal for Numerical Methods in Engineering, 47(12):2039-2072, 2000. 10

[9] L. W. Cordes and B. Moran. Treatment of material discontinuity in the elementfree galerkin method. Computer Methods in Applied Mechanics and Engineering, 139(1-4):75 - 89, 1996. 2, 21

[10] J. C. Costa. Análise linear de cascas com método de galerkin livre de elementos. Master's thesis, Polytechnical School at the University of Sao Paulo, 2010. 4 
[11] J. C. Costa, P. de Mattos Pimenta, and P. Wriggers. Meshless analysis of shear deformable shells: boundary and interface constraints. Computational Mechanics, recently submitted. 57

[12] J. C. Costa, C. M. Tiago, and P. de Mattos Pimenta. Meshless analysis of shear deformable shells: the linear model. Computational Mechanics, 52(4):763 - 778, 2013. $1,2,4,10,12,18$

[13] E. M. de Barreto Campello, P. de Mattos Pimenta, and P. Wriggers. A triangular finite shell element based on a fully nonlinear shell formulation. Computational Mechanics, 31(6):505-518, 2003. 11

[14] P. de Mattos Pimenta and E. M. de Barreto Campello. Shell curvature as an initial deformation: A geometrically exact finite element approach. International Journal for Numerical Methods in Engineering, 78(9):1094-1112, 2009. 2, 4

[15] J. Dolbow and I. Harari. An efficient finite element method for embedded interface problems. International Journal for Numerical Methods in Engineering, 78(2):229 252, 2009. 2, 21, 36, 56

[16] A. Embar, J. Dolbow, and I. Harari. Imposing dirichlet boundary conditions with nitsche's method and spline-based finite elements. International Journal for Numerical Methods in Engineering, 83(7):877 - 898, 2010. 2, 36

[17] S. Fernández-Méndez, J. Bonet, and A. Huerta. Continuous blending of $\{\mathrm{SPH}\}$ with finite elements. Computers \& Structures, 83(17-18):1448 - 1458, 2005. Advances in Meshfree Methods. 20

[18] S. Fernández-Méndez and A. Huerta. Imposing essential boundary conditions in mesh-free methods. Computer Methods in Applied Mechanics and Engineering, 193(12-14):1257 - 1275, 2004. Meshfree Methods: Recent Advances and New Applications. 1, 2, 20, 31, 36

[19] A. Fritz, S. Hüeber, and B. I. Wohlmuth. A comparison of mortar and nitsche techniques for linear elasticity. CALCOLO, 41(3):115-137, 2004. 21

[20] M. Griebel and M. A. Schweitzer. A particle-partition of unity method Part V: Boundary conditions. In Geometric Analysis and Nonlinear Partial Differential Equations. 2,36

[21] Y. Guo and M. Ruess. A layerwise isogeometric approach for NURBS-derived laminate composite shells. Composite Structures, 124:300 - 309, June 2015. 22 
[22] Y. Guo and M. Ruess. Nitsche's method for a coupling of isogeometric thin shells and blended shell structures. Computer Methods in Applied Mechanics and Engineering, 284:881 - 905, Feb. 2015. 2, 22, 51

[23] Y. Guo and M. Ruess. Weak Dirichlet boundary conditions for trimmed thin isogeometric shells. Computers \& Mathematics with Applications, June 2015. 2, 22

[24] A. Hansbo and P. Hansbo. An unfitted finite element method, based on nitsche's method, for elliptic interface problems. Computer Methods in Applied Mechanics and Engineering, 191(47-48):5537-5552, 2002. 21

[25] I. Harari and J. E. Dolbow. Analysis of an efficient finite element method for embedded interface problems. Computational Mechanics, 46(1):205 - 211, 2010. 2

[26] I. Harari and E. Grosu. A unified approach for embedded boundary conditions for fourth-order elliptic problems. International Journal for Numerical Methods in Engineering, pages $\mathrm{n} / \mathrm{a}-\mathrm{n} / \mathrm{a}, 2014.2$

[27] I. Harari and E. Shavelzon. Embedded kinematic boundary conditions for thin plate bending by nitsche's approach. International Journal for Numerical Methods in Engineering, 92(1):99 - 114, 2012. 2, 38

[28] M. Hautefeuille, C. Annavarapu, and J. E. Dolbow. Robust imposition of dirichlet boundary conditions on embedded surfaces. International Journal for Numerical Methods in Engineering, 90(1):40 - 64, 2012. 2, 21

[29] A. Huerta and S. Fernández-Méndez. Enrichment and coupling of the finite element and meshless methods. International Journal for Numerical Methods in Engineering, 48(11):1615-1636, 2000. 2, 20

[30] A. Huerta, S. Fernández-Méndez, and W. K. Liu. A comparison of two formulations to blend finite elements and mesh-free methods. Computer Methods in Applied Mechanics and Engineering, 193(12-14):1105 - 1117, 2004. Meshfree Methods: Recent Advances and New Applications. 20

[31] V. Ivannikov, C. Tiago, and P. M. Pimenta. Meshless implementation of the geometrically exact kirchhoff-love shell theory. International Journal for Numerical Methods in Engineering, 100(1):1 - 39, 2014. 1, 2

[32] V. Ivannikov, C. Tiago, and P. M. Pimenta. On the boundary conditions of the geometrically nonlinear kirchhoff-love shell theory. International Journal of Solids and Structures, 51(18):3101 - 3112, 2014. 2 
[33] W. Jiang, C. Annavarapu, J. E. Dolbow, and I. Harari. A robust nitsche's formulation for interface problems with spline-based finite elements. International Journal for Numerical Methods in Engineering, 2015. 2

[34] L. A. B. Jr., O. L. Manzoli, P. G. Prazeres, E. A. Rodrigues, and T. N. Bittencourt. A coupling technique for non-matching finite element meshes. Computer Methods in Applied Mechanics and Engineering, 290:19 - 44, 2015. 21

[35] Y. Krongauz and T. Belytschko. Enforcement of essential boundary conditions in meshless approximations using finite elements. Computer Methods in Applied Mechanics and Engineering, 131(1-2):133 - 145, 1996. 1, 20

[36] Y. Krongauz and T. Belytschko. Efg approximation with discontinuous derivatives. International Journal for Numerical Methods in Engineering, 41(7):1215 - 1233, 1998. 21

[37] P. Krysl and T. Belytschko. Analysis of thin shells by the element-free galerkin method. International Journal of Solids and Structures, 33(20-22):3057 - 3080, 1996. 1

[38] S. Li, W. Hao, and W. K. Liu. Numerical simulations of large deformation of thin shell structures using meshfree methods. Computational Mechanics, 25(2-3):102-116, 2000. 1

[39] S. Li and W. K. Liu. Meshfree Particle Methods. Springer, Berlin, 2004. 2, 16

[40] G. R. Liu. Mesh Free methods: Moving beyond the finite element method. CRC Press, 2010. 16

[41] G. R. Liu and Y. T. Gu. An Introduction to Meshfree Methods and Their Programming. Springer Netherlands, Dordrecht, 2005. 18, 20

[42] Y. Lu, T. Belytschko, and L. Gu. A new implementation of the element free galerkin method. Computer Methods in Applied Mechanics and Engineering, 113(3-4):397 414, 1994. 2, 20, 33

[43] Y. Lu, T. Belytschko, and M. Tabbara. Element-free galerkin method for wave propagation and dynamic fracture. Computer Methods in Applied Mechanics and Engineering, 126(1-2):131 - 153, 1995. 2, 20

[44] MATLAB. version 8.5.0 (R2015a). The MathWorks Inc., Natick, Massachusetts, 2015. 24 
[45] B. Nayroles, G. Touzot, and P. Villon. Generalizing the finite element method: Diffuse approximation and diffuse elements. Computational Mechanics, 10(5):307-318, 1992. 16,22

[46] V. P. Nguyen, P. Kerfriden, M. Brino, S. P. A. Bordas, and E. Bonisoli. Nitsches method for two and three dimensional nurbs patch coupling. Computational Mechanics, 53(6):1163 - 1182, 2014. 2, 31, 36

[47] J. Nitsche. Über ein variationsprinzip zur lösung von dirichlet-problemen bei verwendung von teilräumen, die keinen randbedingungen unterworfen sind. Abhandlungen aus dem Mathematischen Seminar der Universität Hamburg, 36(1):9-15, 1971. 2, 20, 31

[48] H. Noguchi, T. Kawashima, and T. Miyamura. Element free analyses of shell and spatial structures. International Journal for Numerical Methods in Engineering, 47(6):1215-1240, 2000. 1

[49] M. Ruess, D. Schillinger, Y. Bazilevs, V. Varduhn, and E. Rank. Weakly enforced essential boundary conditions for NURBS-embedded and trimmed NURBS geometries on the basis of the finite cell method. International Journal for Numerical Methods in Engineering, 95(10):811 - 846, Sept. 2013. 2, 21, 33, 36

[50] M. Ruess, D. Schillinger, A. I. Özcan, and E. Rank. Weak coupling for isogeometric analysis of non-matching and trimmed multi-patch geometries. Computer Methods in Applied Mechanics and Engineering, 269:46-71, Feb. 2014. 2, 21, 36

[51] E. Sala-Lardies, S. Fernández-Méndez, and A. Huerta. Optimally convergent highorder x-fem for problems with voids and inclusions. pages 8080-8093, 2012. 2

[52] J. D. Sanders, J. E. Dolbow, and T. A. Laursen. On methods for stabilizing constraints over enriched interfaces in elasticity. International Journal for Numerical Methods in Engineering, 78(9):1009 - 1036, 2009. 2, 36, 38

[53] J. D. Sanders, T. A. Laursen, and M. A. Puso. A nitsche embedded mesh method. Computational Mechanics, 49(2):243 - 257, 2012. 2, 21, 32, 36

[54] J. D. Sanders and M. A. Puso. An embedded mesh method for treating overlapping finite element meshes. International Journal for Numerical Methods in Engineering, 91(3):289 - 305, 2012. 2

[55] J. Simo and T. Hughes. Computational Inelasticity. Interdisciplinary applied mathematics: Mechanics and materials. Springer, 1998. 11 
[56] J. C. Simo, D. D. Fox, and M. S. Rifai. On a stress resultant geometrically exact shell model. part ii: The linear theory; computational aspects. Computer Methods in Applied Mechanics and Engineering, 73(1):53 - 92, 1989. 49

[57] R. Stenberg. On some techniques for approximating boundary conditions in the finite element method. Journal of Computational and Applied Mathematics, 63(1-3):139 148, 1995. Proceedings of the International Symposium on Mathematical Modelling and Computational Methods Modelling 94. 2

[58] C. M. Tiago. Meshless methods: Extending the linear formulation and its generalization to geometrically exact structural analysis. PhD thesis, Instituto Superior Técnico, Universidade Técnica de Lisboa, 2007. 1, 2, 16, 18, 33

[59] P. Wriggers and G. Zavarise. A formulation for frictionless contact problems using a weak form introduced by nitsche. Computational Mechanics, 41(3):407-420, 2008. 2

[60] T. Zhu and S. N. Atluri. A modified collocation method and a penalty formulation for enforcing the essential boundary conditions in the element free galerkin method. Computational Mechanics, 21(3):211-222, 1998. 27 